BORESCOPIC LASER DOPPLER VELOCIMETRY PROBE

\author{
by \\ KORY T. O'BRIEN \\ SEMIH M. OLCMEN, COMMITTEE CHAIR \\ JOHN BAKER \\ BRIAN T. FISHER
}

\begin{abstract}
A THESIS
Submitted in partial fulfillment of the requirements for the degree of Master of Science in the Department of Aerospace Engineering and Mechanics in the Graduate School of

The University of Alabama
\end{abstract}

TUSCALOOSA, ALABAMA

2013 
Copyright Kory Thomas O'Brien 2013

ALL RIGHTS RESERVED 


\begin{abstract}
A miniature fiber-optic, single-velocity-component Laser Doppler Velocimetry (LDV) probe for measurement in cramped spaces, where access is very limited, has been designed, constructed, and tested. The probe design allows the main probe dimensions to be small $(7 \mathrm{~mm}$ in diameter). In addition, the proposed back-scatter collection scheme allows the main section to be as long as needed to access remote locations. The laser beams are first collimated by passing them through two separate collimating lenses. The collimated light then passes through $1 \mathrm{~mm}$ holes machined into a right angle prism-mirror and are focused to form the measurement probe volume using the focusing lens placed at the end of the probe extension tube. The light scattered by the particles in the flow is collected back by the focusing lens and is collimated. The collimated light then reflects off the right-angle mirror by 90 degrees, passes through the receiving lens, and is focused to the receiving fiber terminator. The receiving fiber-optic cable transmits the collected light to the photo-multiplier tube which then converts the signal into an electrical signal for further processing of the data. The probe working principle was proven in two types of jet flows.
\end{abstract}




\section{LIST OF ABBREVIATIONS AND SYMBOLS}

\begin{tabular}{|c|c|c|c|}
\hline$\vec{e}_{b}$ & $\begin{array}{l}\text { Unit vector along the incident beam } \\
\text { direction }\end{array}$ & $\mathrm{f}$ & Focal length of lens \\
\hline$\vec{e}_{p r}$ & $\begin{array}{l}\text { Unit vector in the direction of the } \\
\text { observer from the particle }\end{array}$ & $f_{b}$ & Frequency of laser light \\
\hline$\vec{v}_{p}$ & Velocity of particle & $f_{D}$ & Doppler frequency \\
\hline$\overline{\mathrm{U}}$ & Mean velocity component & FDP & Frequency-domain processor \\
\hline$\Delta \delta$ & Phase difference & $f_{p}$ & $\begin{array}{l}\text { Frequency of light scattered by } \\
\text { particle }\end{array}$ \\
\hline$\Delta \rho$ & Particle and fluid density difference & $\mathrm{f}_{\mathrm{r}}$ & $\begin{array}{l}\text { Particle frequency received by } \\
\text { stationary detector }\end{array}$ \\
\hline $\mathrm{a}$ & Particle diameter & FSA & Flow-size analyzer \\
\hline AMP & Amplifier & $\mathrm{f}_{\mathrm{sh}}$ & Shift frequency \\
\hline $\mathrm{BC}$ & Bragg cell & $\mathrm{g}_{\mathrm{c}}$ & Gravitational acceleration \\
\hline $\mathrm{c}$ & Speed of light & ICE & Internal combustion engine \\
\hline $\mathrm{c}_{\mathrm{d}}$ & $\begin{array}{l}\text { Particle Cunningham slip } \\
\text { correction factor }\end{array}$ & LCU & Light collection unit \\
\hline $\mathrm{c}_{\mathrm{da}}$ & $\begin{array}{l}\text { Idealized particle Cunningham slip } \\
\text { correction factor }\end{array}$ & LDV & Laser Doppler Velocimetry \\
\hline$d_{a}$ & Aerodynamic diameter & $1_{\mathrm{m}}$ & Length of measurement volume \\
\hline$D_{e}^{-2}$ & Diameter of laser beam & LTFC & Laser-to-fiber coupler \\
\hline$d_{f}$ & Fringe spacing & MIX & Mixer \\
\hline$d_{m}$ & Diameter of measurement volume & $\mathrm{n}_{\mathrm{e}}$ & Extraordinary wave \\
\hline
\end{tabular}




\begin{tabular}{|c|c|c|c|}
\hline $\mathrm{N}_{\mathrm{fr}}$ & Number of fringes & $\mathrm{V}_{\mathrm{fi}}$ & Initial fluid velocity \\
\hline $\mathrm{n}_{\mathrm{o}}$ & Ordinary wave & $\mathrm{V}_{\mathrm{p}}$ & Particle velocity \\
\hline $\mathrm{N}_{\mathrm{p}}$ & Particle concentration & $\mathrm{V}_{\mathrm{pi}}$ & Initial particle velocity \\
\hline $\mathrm{N}_{\mathrm{s}}$ & Seeder particle concentration & $\mathrm{V}_{\mathrm{ps}}$ & Particle settling velocity \\
\hline PMT & Photo-multiplier tube & $\alpha$ & $\begin{array}{l}\text { Angle between shifted beam and } \\
\text { non-shifted beam }\end{array}$ \\
\hline $\mathrm{Q}_{\mathrm{s}}$ & Seeder volumetric flow rate & $\alpha_{b}$ & Divergence angle \\
\hline $\mathrm{Q}_{\mathrm{T}}$ & Total volumetric flow rate & $\eta$ & Relative refractive index \\
\hline RFG & Radio frequency generator & $\lambda$ & Wavelength of laser light \\
\hline SNR & Signal-to-noise ratio & $\Lambda_{\mathrm{ac}}$ & Acoustic wavelength \\
\hline $\mathrm{t}$ & time & $\mu_{\mathrm{g}}$ & Fluid dynamic viscosity \\
\hline TKE & Turbulent kinetic energy & $\rho_{\mathrm{p}}$ & Particle density \\
\hline $\mathrm{u}$ & Fluctuating velocity & $\sigma_{\mathrm{n}}$ & Noise fluctuations \\
\hline $\mathrm{U}$ & Velocity component & $\sigma_{\mathrm{s}}$ & Signal fluctuations \\
\hline $\mathrm{V}$ & Volume of measurement volume & $\tau$ & Relaxation time \\
\hline $\mathrm{V}_{\mathrm{a}}$ & Acoustic velocity & & \\
\hline$V_{\mathrm{f}}$ & Fluid velocity & & \\
\hline
\end{tabular}




\section{ACKNOWLEDGMENTS}

I would like to first thank Dr. Semih Olcmen, my project director and committee chair, for his guidance on this project and for taking me in as a research assistant on such late notice my senior year. This project would not have been completed if not for all the long hours he put in with me over the summer. I would also like to thank Dr. John Baker and Dr. Brian Fisher for serving on my thesis committee. I would like to acknowledge the engineering machine shop in the design and construction of the components of the probe. Finally, I would like to thank The University of Alabama and the Department of Aerospace Engineering for the financial support they provided me with as a graduate student. 


\section{CONTENTS}

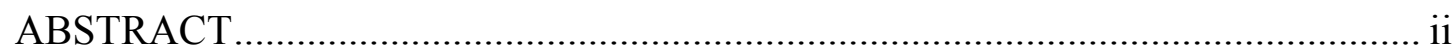

LIST OF ABBREVIATIONS AND SYMBOLS ............................................. iii

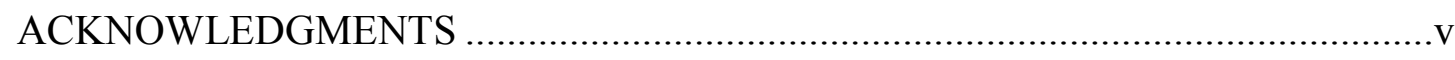

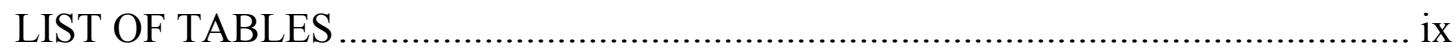

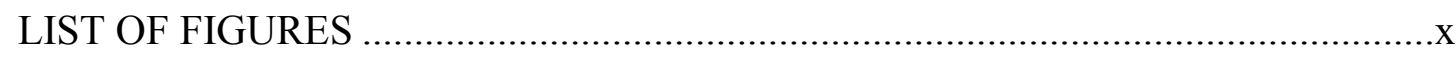

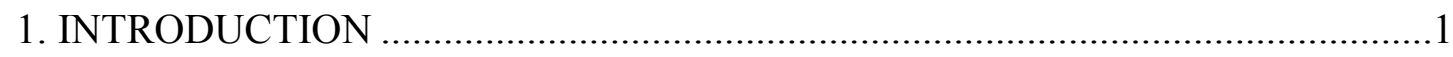

1.1 LASER DOPPLER VELOCIMETRY ..........................................

1.2 BORESCOPIC LDV PROBE ......................................................2

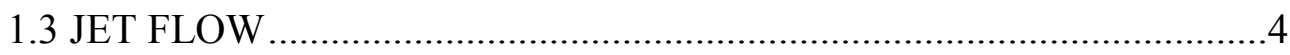

2. FLOW DECRIPTION ..............................................................................

2.1 REGIONS OF FLOW .................................................................

2.1.1 REGION I .................................................................

2.1.2 REGION II.................................................................

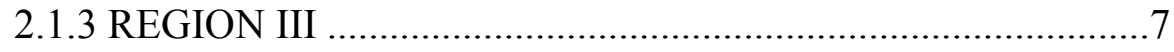

2.2 CENTERLINE JET DECAY RELATIONS ......................................8

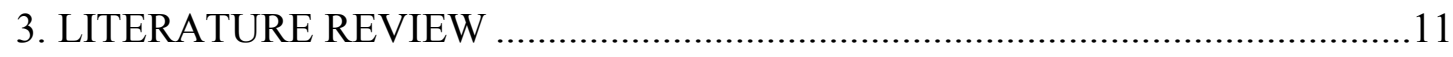

3.1 MINIATURE LDV PROBES ................................................. 11

3.2 PIPE JET FLOW ........................................................................ 11

3.3 CONVERGENT NOZZLE JET FLOW ..........................................14

4. LASER DOPPLER VELOCIMETRY TECHNIQUE …..................................17

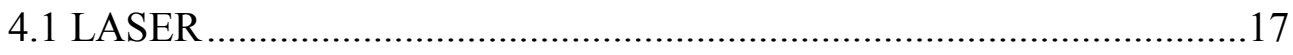




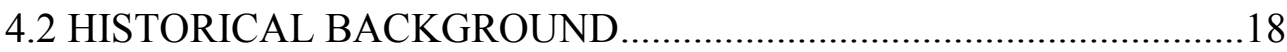

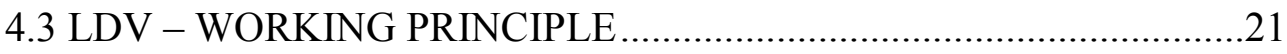

4.4 MEASUREMENT VOLUME ……........................................................25

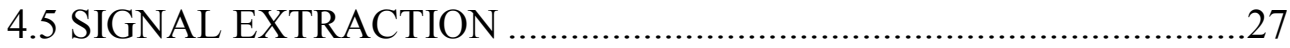

4.6 TURBULENCE PARAMETERS AND MEASUREMENT...................29

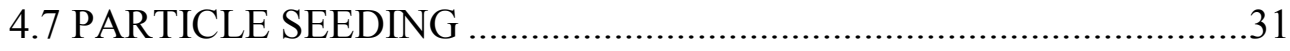

5. PROBE DESIGN AND CONSTRUCTION ………….........................................

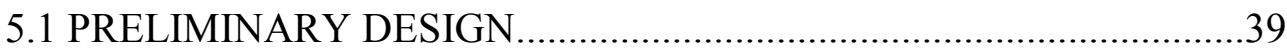

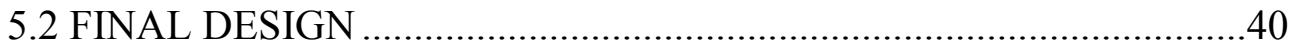

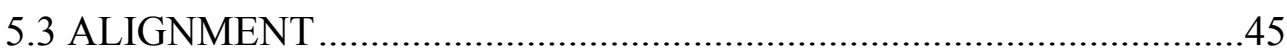

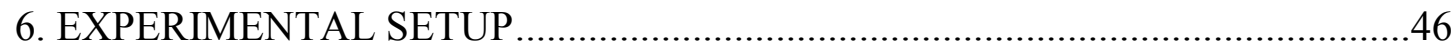

6.1 COMPONENTS OF AN LDV SYSTEM ……………………..........4

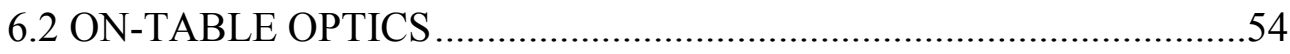

6.3 DATA ACQUISITION AND REDUCTION UNITS …………..............55

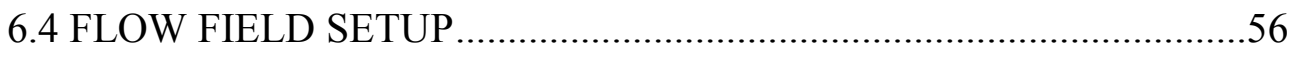

6.4.1 PARTICLE SEEDING GENERATOR …………………….....56

6.4.2 MANOMETER \& BERNOULLI'S EQUATION ……….........57

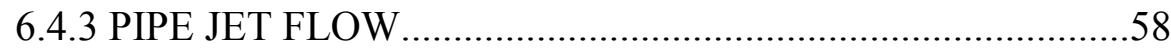

6.4.4 CONVERGENT NOZZLE JET FLOW ..................................61

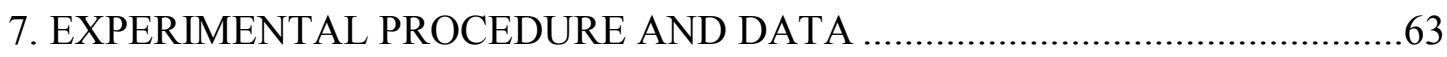

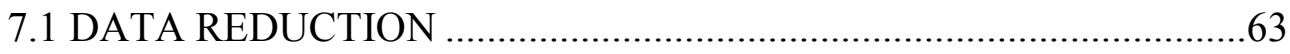

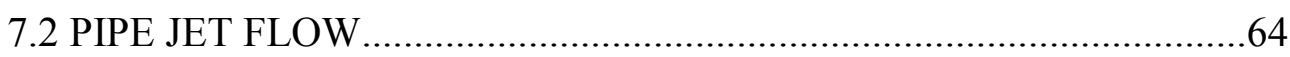

7.3 CONVERGENT NOZZLE FLOW …………………………................. 
7.4 UNCERTAINTIES .................................................................. 74

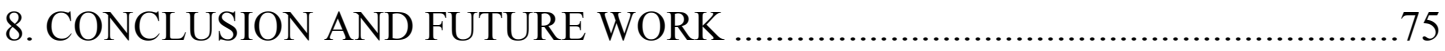

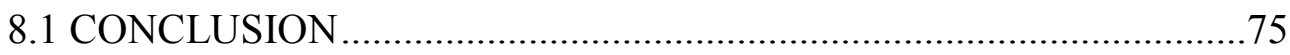

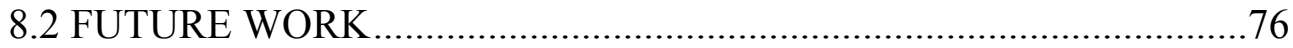

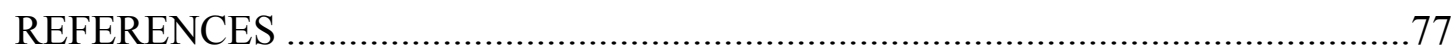

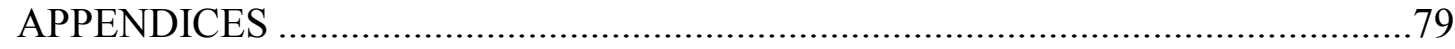




\section{LIST OF TABLES}

2.1 Spreading rate $S$ and velocity-decay constant $B$ for turbulent round jets.............10

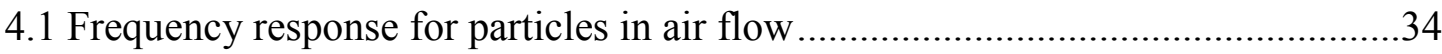

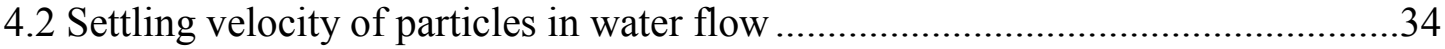

5.1 Purchased LDV probe components price list................................................41

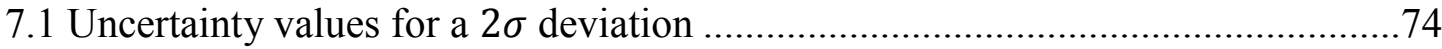




\section{LIST OF FIGURES}

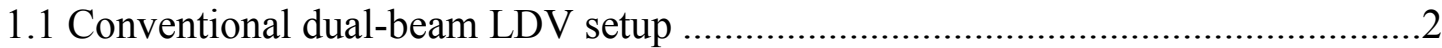

1.2 Borescope

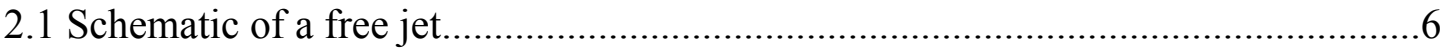

2.2 Axial velocity distributions for turbulent round jet ...............................................

2.3 Mean axial velocity versus radial distance for a turbulent jet ................................8

2.4 Variation of centerline mean axial velocity with axial distance .............................9

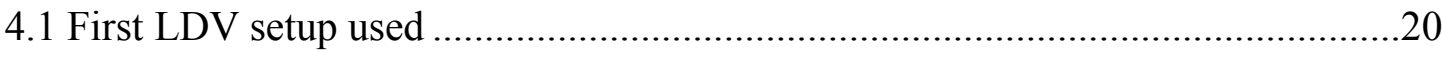

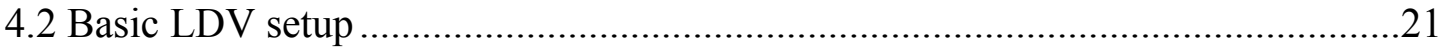

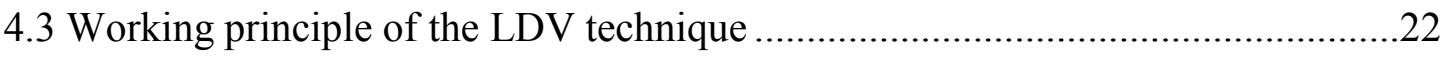

4.4 LDV - Intersecting beams and vector relations ...................................................23

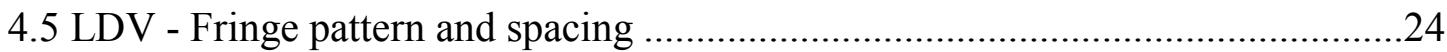

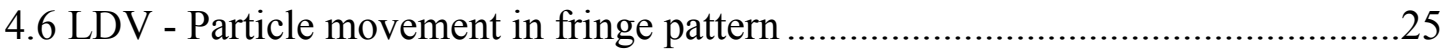

4.7 Distortion of fringes at measurement volume.....................................................26

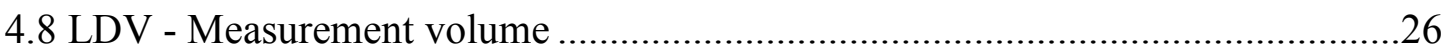

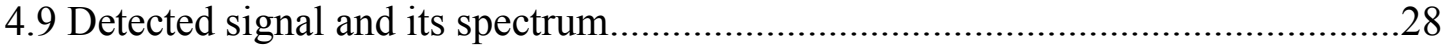

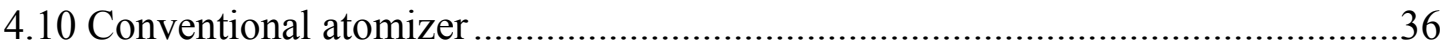

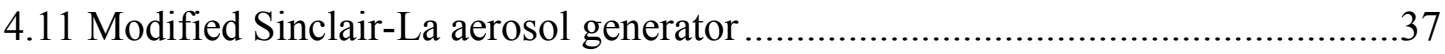

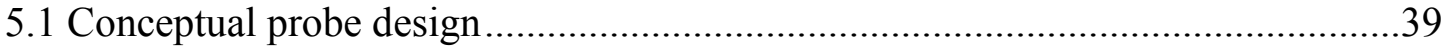

5.2 Main probe structure block ………….................................................................41

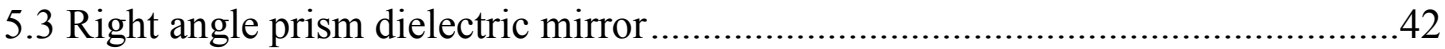




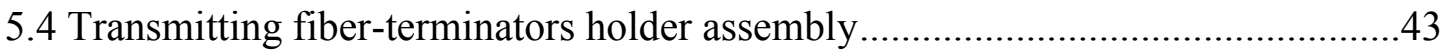

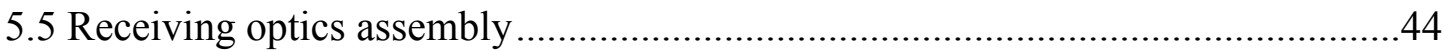

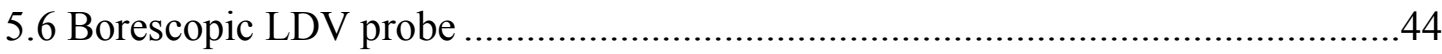

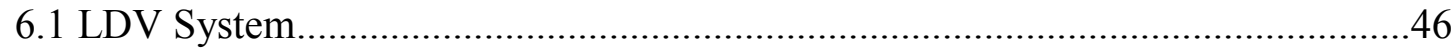

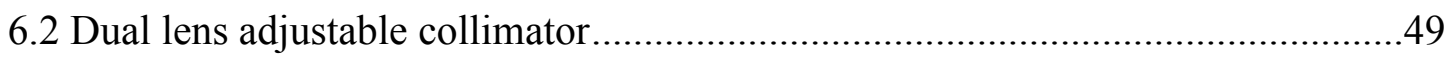

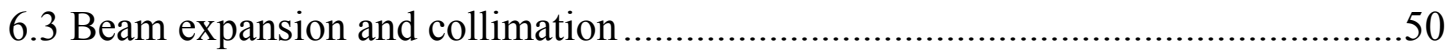

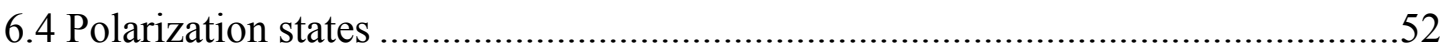

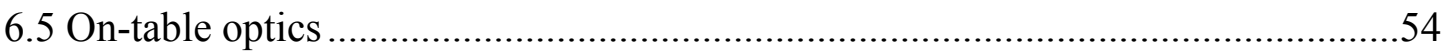

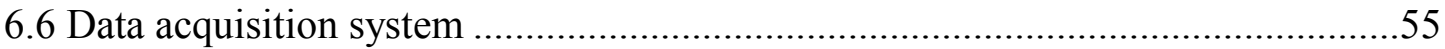

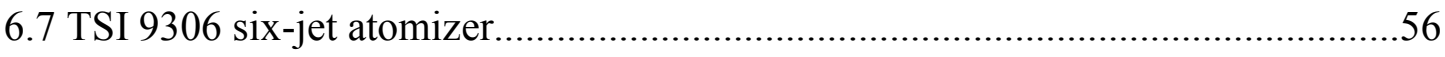

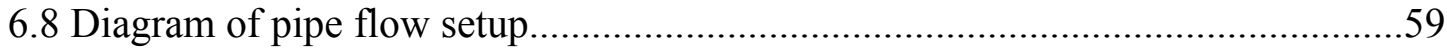

6.9 TSI Model 1125 hot-wire anemometry calibrator .......................................61

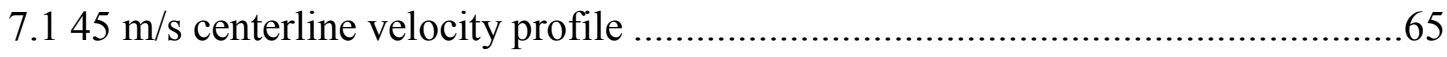

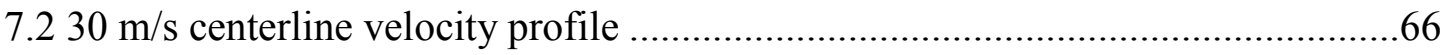

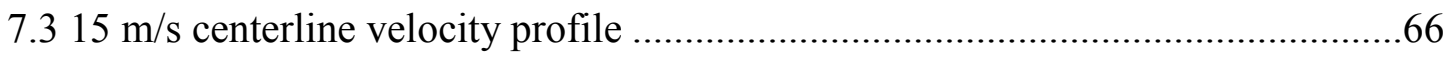

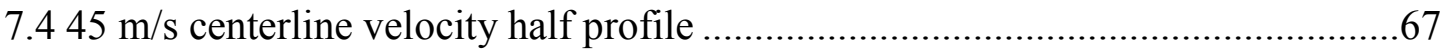

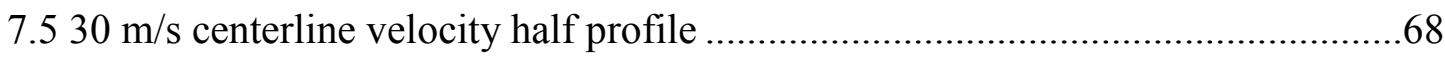

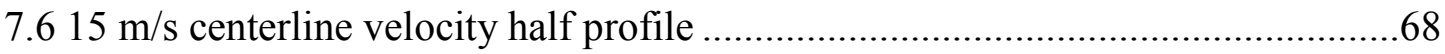

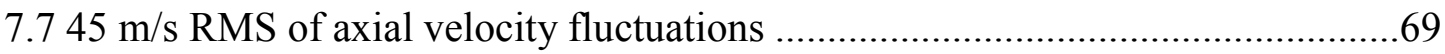

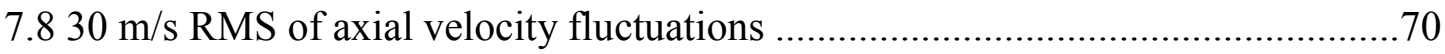

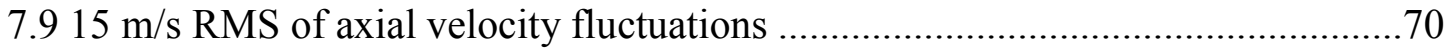

7.10 Velocity decay of $75 \mathrm{~m} / \mathrm{s}$ convergent nozzle flow ..................................... 71

7.11 RMS of axial velocity fluctuations of centerline velocity decay .......................72 
7.12 Variation of the mean axial velocity along the centerline of the jet. .73

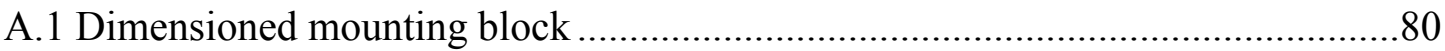

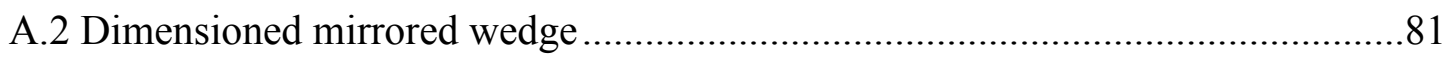

A.3 Dimensioned long-neck probe head tube ................................................................ 81

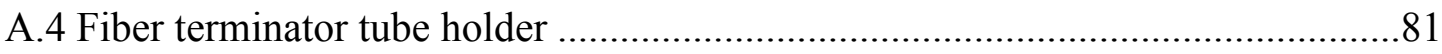

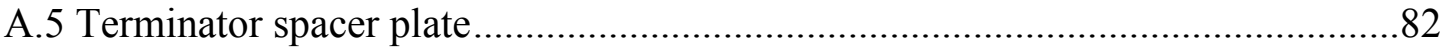

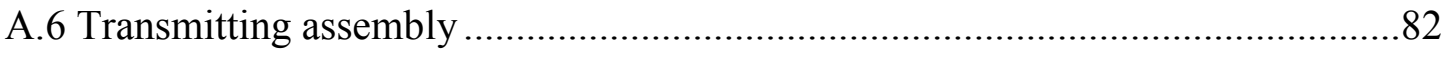

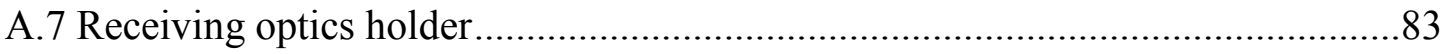

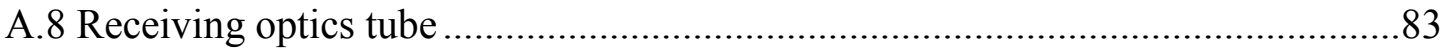

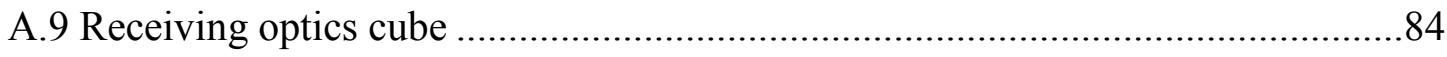

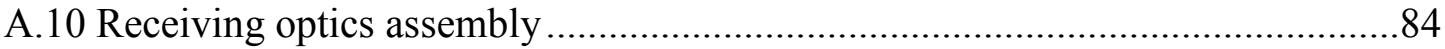

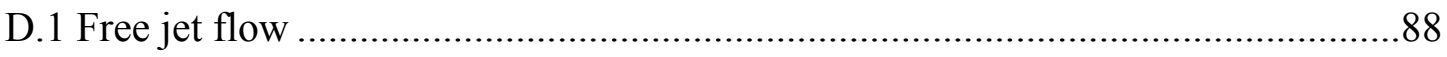

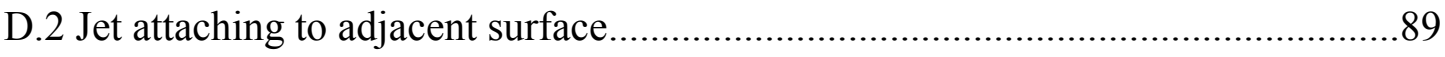

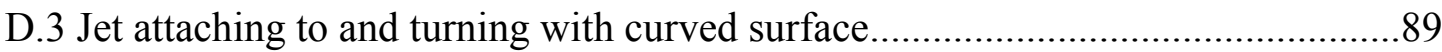




\section{INTRODUCTION}

\subsection{LASER DOPPLER VELOCIMETRY}

Laser Doppler Velocimetry (LDV) is powerful and widely used for fluid flow studies. The main advantages of LDV are that, i) it is a non-invasive measurement technique to measure flow properties and, ii) it has high spatial resolution. LDV allows non-intrusive velocity and turbulence measurements by accessing the flow through small optical windows where such measurements would otherwise be extremely difficult. One of the biggest disadvantages is that it is a point measurement technique.

The LDV technique uses the Doppler Effect to measure flow velocity at a point. The Doppler Effect uses the principle that the frequency of a wave emitted by a moving object, as observed by a stationary observer, changes as a function of velocity.

As a source approaches an observer, the intervals between waves change resulting in an increase in frequency. As the source retreats, the intervals' increase and a decrease in frequency is observed. By determining the frequency change, one can determine whether the source is advancing or retreating. The total Doppler Effect results from either the movement of the source or movement of the observer. 
The scattered light's frequency changes as the particle moves and is proportional to $\frac{2 V_{p}}{c}$ where $V_{p}$ is the particle's velocity and $c$ is the speed of light. Since the speed of light is several orders of magnitude larger than the particle's velocity, the small frequency change is not easily detected. In order to circumvent this problem, scattered light is mixed with the unscattered light from the laser beam. Beat frequencies that originate due to the Doppler shift are then measured.

For the conventional dual-beam LDV technique, two mutually coherent laser beams intersect with each other after passing through a focusing lens. This crossing point is called the measurement probe volume. The light scattered by the particles is collected by a receiving lens, which focuses the scattered light onto a photo detector. The signal from the photo detector is then amplified and analyzed by a signal processor. This basic LDV setup is shown in Figure 1.1

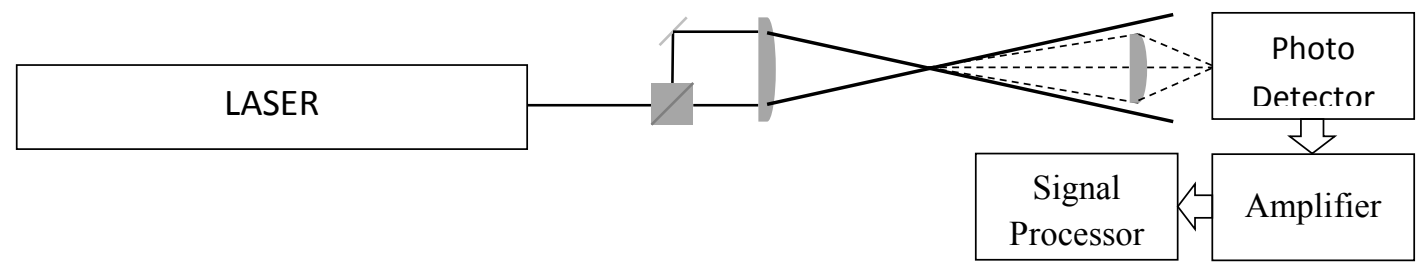

Figure 1.1 Conventional dual-beam LDV setup.

\subsection{BORESCOPIC LDV PROBE}

The LDV probe was designed to look and perform similar to a borescope (Figure 1.2). The idea behind the research was to make the probe head diameter as small as possible with a long neck such that it could be inserted into cramped spaces where conventional probes cannot be used such as deep within a car engine where space is limited. The probe contains the transmitting and receiving fibers in one physical probe similar to conventional backscatter LDV 
probes. The probe is also designed for velocity measurements $30 \mathrm{~mm}$ or $60 \mathrm{~mm}$ away from the probe tip based on the transmitting/receiving lens focal length used.

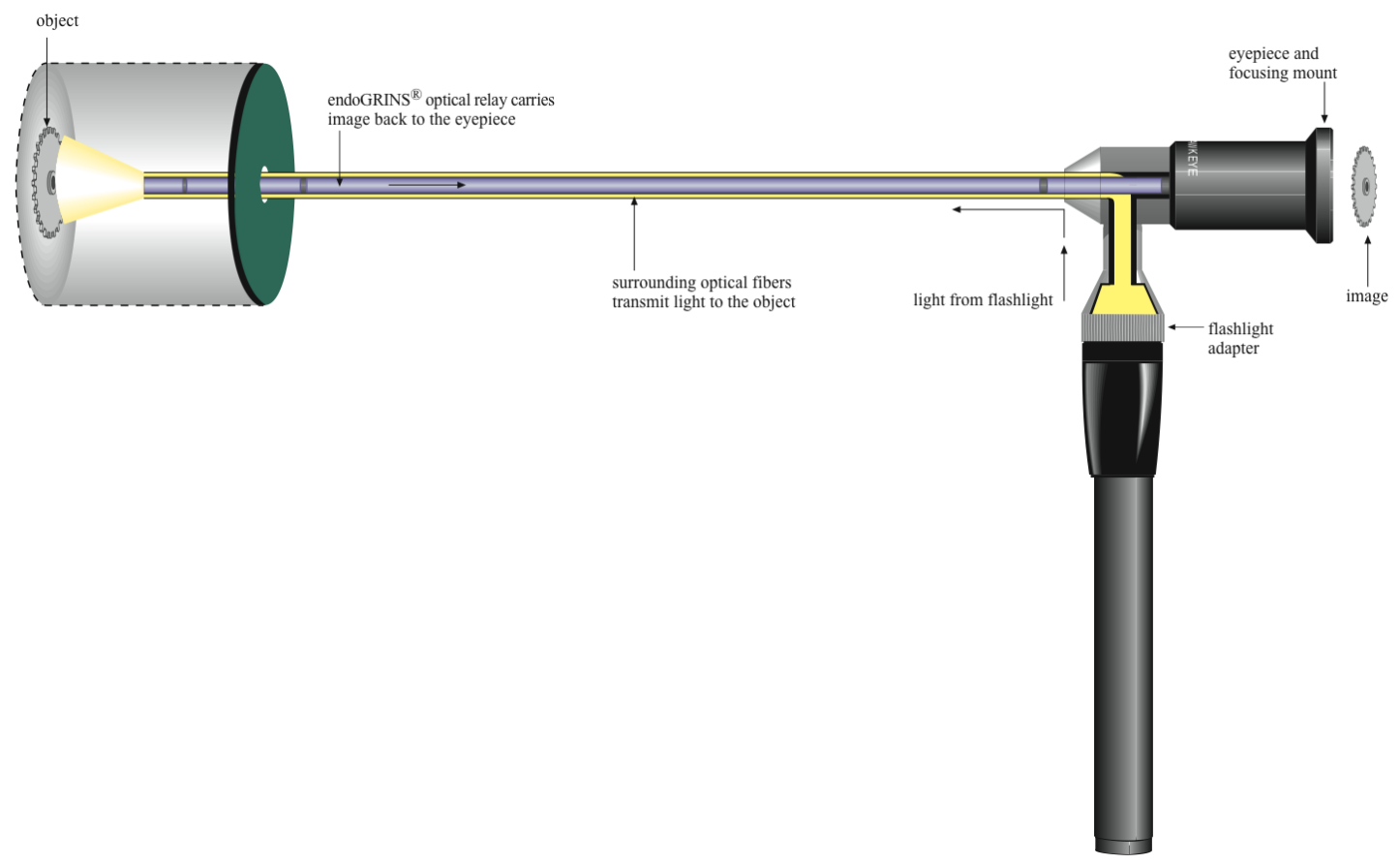

Figure 1.2 Borescope.

All of the components of the probe, with the exception of the lenses, prism, and fibers, are stainless steel and were either purchased or machined. The laser beams travel through the fiber-optic cables and end at fiber terminators inserted into tubes that are separated by a small separating plate which are then inserted into a larger tube (Figure 5.4). The beams, emerging from the fiber terminators then pass through lenses, separately, that collimate the beams into two parallel beams. The collimated beams pass through holes drilled through the right angle prism/mirror located within the block that holds the probe together. The beams then pass through the longneck probe head extension tube and through the focusing lens. The crossing of the focused beams was observed by passing the beams though a microscope objective placed at the focal point of the focusing lens. The beams were then made to overlap on top of each other by manipulating the fiber terminators. Once the alignment was complete, all movable parts were 
glued into place using cyanoacrylate glue. The microscope objective was then removed and the point where the beams overlapped formed the probe volume. Light scattered by the particles in the flow are collected back through the focusing lens, working as the receiving lens, which recollimates the collected light. The collimated, collected light travels back to the right angle mirror and reflects by $90^{\circ}$ through the receiving lens where it is focused on to the receiving fiber. The receiving fiber was adjusted using adjustment screws to capture the reflected light. The focused light is then carried through the multi-mode receiving fiber-optic cable to the photomultiplier tube for further data acquisition and reduction.

\subsection{JET FLOW}

The probe was tested in two different jet flows to demonstrate that it could measure velocities accurately. Different tests were performed on each flow to show that the probe worked correctly. The two jet flows used for this demonstration were jets from a $40.89-\mathrm{mm}$ (1.61-in) diameter pipe and from a 3.81-mm (0.15-in) diameter converging nozzle.

For the pipe jet, the axial velocity component was measured across the symmetry axis of the pipe at varying jet-center velocities set at the jet exit plane. This experiment was performed at jet-exit plane center velocities of $15-\mathrm{m} / \mathrm{s}$ (33.6-mph), 30-m/s $(67.1-\mathrm{mph})$, and $45-\mathrm{m} / \mathrm{s}$ (100.7-mph).

For the converging nozzle jet, the $\mathrm{U}$ component of velocity was measured along the centerline of the nozzle at varying distances away from the exit to obtain the jet's centerline velocity decay. This was performed at $75-\mathrm{m} / \mathrm{s}$ (168-mph). 


\section{FLOW DESCRIPTION}

A fluid jet is formed when discharge occurs from a confining flow passage. A jet is considered a free jet if it is issued into an ambient fluid at rest or into an infinite environment. Common examples of turbulent jets include drinking fountains, drain flows into rivers, and chimney discharges into the atmosphere (Kassab et al., 1996).

One of the most important parameters used to define the behavior of jet flows is Reynolds number, Re. Equation 2.1 defines the non-dimensionalized flow parameter.

$$
R e=\frac{U d}{v}
$$

where $U$ is the velocity at the jet exit, $d$ is the diameter of the exit, and $v$ is the kinematic viscosity of the fluid.

Vickers (1959) describes a jet flow as turbulent when the exit Reynolds number based on the average velocity and the jet diameter is larger than 3000 . For the purpose of this thesis, the jets will all be classified as turbulent as the Reynolds numbers for the jets used are all greater

than $10^{4}$. Turbulent jets are defined by fluctuations that occur in the velocity about a mean velocity value at any point in the flow. 


\subsection{REGIONS OF JET FLOW}

Jet flows can be divided in to different regions where different flow phenomena occur. Figure 2.1 shows a diagram of the flow field of a jet divided into three main regions that are well defined in literature.

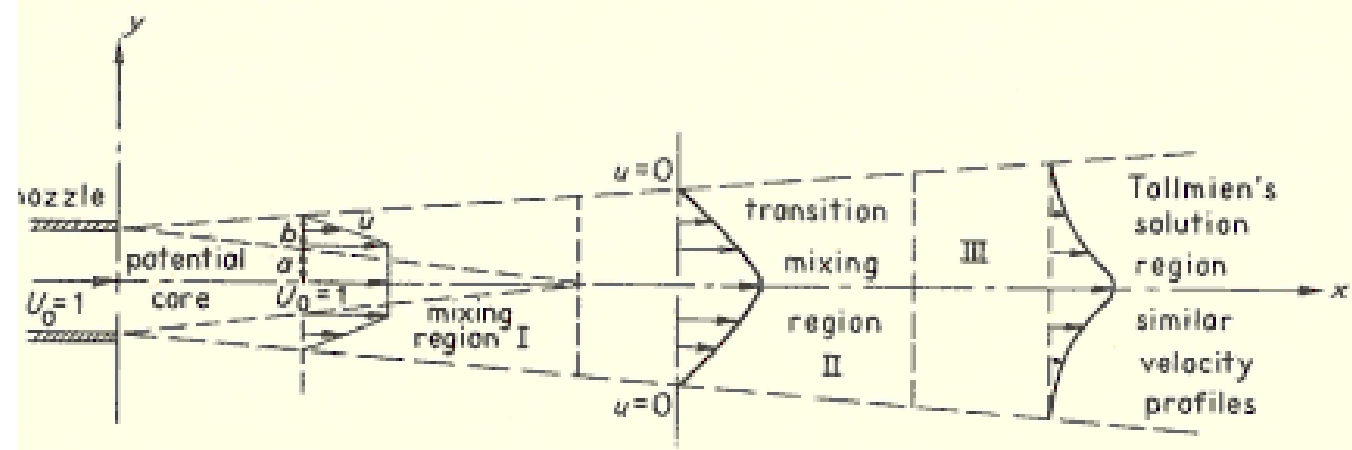

Figure 2.1 Schematic of a free jet (Pai, 1954).

\subsubsection{REGION I}

The initial region of a jet, shown as Region I in Figure 2.1, contains a section known as the potential core. Due to the uniform nature of the velocity at the jet exit, a conical shape extends outward equal to the jet exit velocity; thus, until the end of the potential core, the centerline velocity does not change. The region has been shown to have negligible shear forces and is often modeled as inviscid flow. The exact length of the potential core has been shown to vary between 4.7 and 7.7 jet exit diameters (Hrycak et al., 1970).

\subsubsection{REGION II}

Two main flow parameters change as the distance traveled from the jet exit grows: (i) the jet width increases and, (ii) the centerline velocity of the jet decreases. The mean axial velocity profile shape also changes in this section. The velocity profile has a flattened peak at the center 
due to the presence of the potential core and the velocity approaches zero at the edges of the jet. The flow transitions to fully developed flow in region II once the jet is out of the potential core region.

\subsubsection{REGION III}

Region III is the most studied region of turbulent flow. From the beginning of this region onward, every axial velocity profile downstream has a self-similar Gaussian-like shape. The region generally begins at an $\mathrm{x} / \mathrm{D}=25-30$. Figure 2.2 shows an example of three different velocity distributions occurring in this region using data obtained from Hussein et al. (1994).

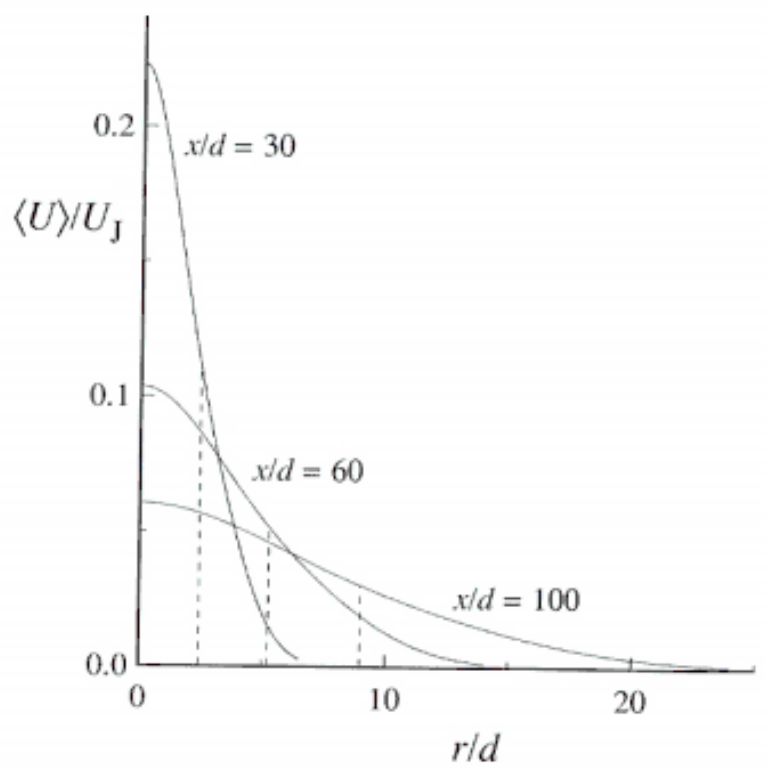

Figure 2.2 Axial velocity distributions for turbulent round jet (Pope, 2000).

(Data obtained from Hussein et al., 1994)

The axial velocity and radial distance in region III are commonly non-dimensionalized by the centerline velocity at that point and the half-velocity width respectively. The half-velocity width is defined as the distance from the centerline to the location where the mean velocity value is half of the centerline velocity. The mean velocity profiles then collapse onto a single profile. 
The flow in region III is thus described as self-similar. Figure 2.3 , created using data from Wyganski and Fiedler (1969), shows this self-similarity for five different axial locations obtained in a flow with Reynolds number around $10^{5}$.

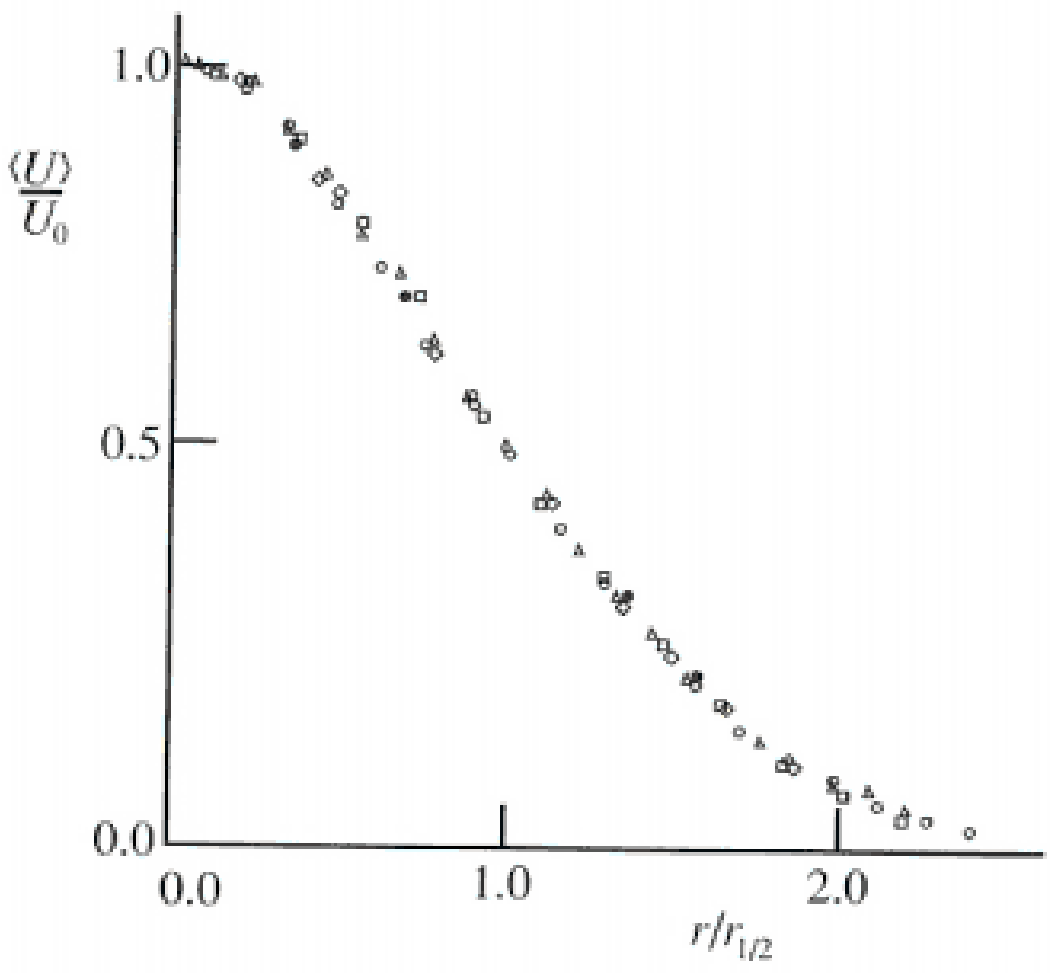

Figure 2.3 Mean axial velocity versus radial distance for a turbulent jet $\circ, \mathrm{x} / \mathrm{d}=40 ; \Delta, \mathrm{x} / \mathrm{d}=60 ; \diamond, \mathrm{x} / \mathrm{d}=60 ; \bullet, \mathrm{x} / \mathrm{d}=97.5($ Pope, 2000).

(Data obtained from Wyganski and Fiedler, 1969)

\subsection{CENTERLINE JET DECAY RELATIONS}

The centerline velocity, $U_{0}$, and the half-velocity jet width, $r_{1 / 2}$, seen in Figure 2.1 are two important variables in turbulent jets. Earlier research has shown these two variables to vary linearly with the distance from the jet exit in regions II and III; this is especially true in region III. The centerline velocity varies with the inverse of the distance from the jet as shown in Equation 2.2 (Pope, 2000) 


$$
\frac{U_{0}(x)}{U_{J}}=\frac{B}{\left(x-x_{0}\right) / d}
$$

where $U_{0}(x)$ is the distribution of the centerline axial velocity, $U_{J}$ is the velocity at the jet exit, $B$ is the empirical constant found from experimental data, $x$ is the axial distance, $x_{0}$ is the virtual origin also found from empirical data, and $d$ is the jet diameter. Data measured by Hussein et al. (1994) was fit to a curve as shown in Figure 2.4 to determine the values of $B$ and $x_{0} / d$ in Equation 2.2. The data corresponds to $x_{0} / d=4$ and $B=5.8$.

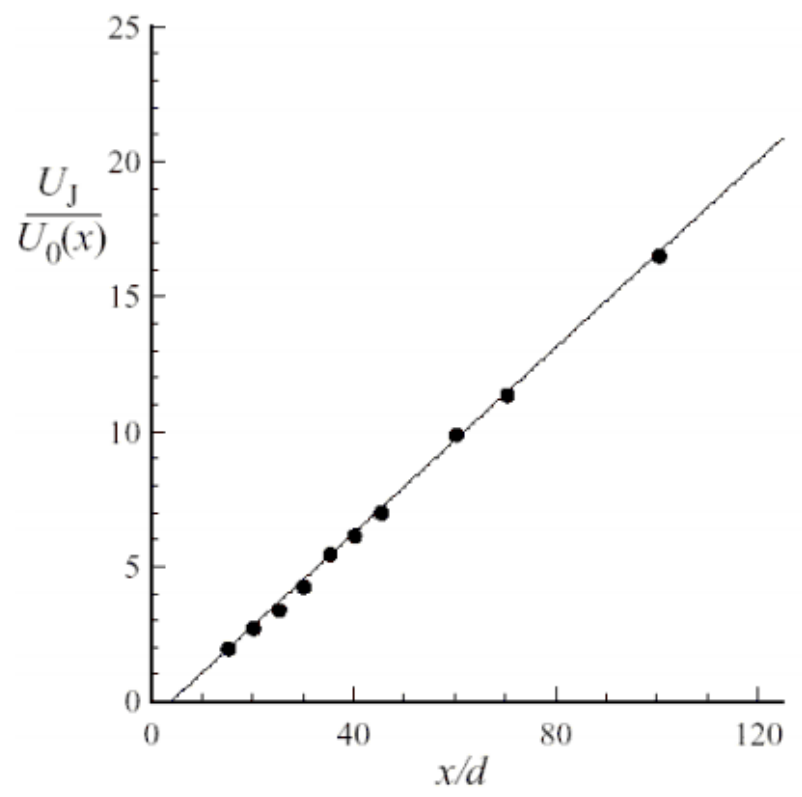

Figure 2.4 Variation of centerline mean axial velocity with axial distance at $\mathrm{Re}=95,500$ (Pope, 2000).

(Data obtained from Hussein et al., 1994)

Equation 2.2 is not valid for the potential core region of the jet. The centerline velocity must equal the jet velocity at the jet exit, or $U_{J} / U_{0}=1$. For this reason, below $x / d=20$, the curve shown in Figure 2.4 will not be linear as shown. 
A similar empirical relation exists with the jet half-width being a function of axial distance from the jet exit as given by Equation 2.3 (Pope, 2000). The equation, however, is only valid in the fully developed region of the flow.

$$
r_{1 / 2}(x)=S\left(x-x_{0}\right)
$$

$S$ and $B$ from the above relationships were found by fitting curves to three sets of experimental results. The resulting values of $S$ and $B$ are shown in Table 2.1 below.

Table 2.1 Spreading rate S and velocity-decay constant B for turbulent round jets (Pope, 2000).

\begin{tabular}{|l|c|c|c|}
\hline & $\begin{array}{c}\text { Panchapakesan and } \\
\text { Lumley (1993) }\end{array}$ & $\begin{array}{c}\text { Hussein et al. hot-wire } \\
\text { data (1994) }\end{array}$ & $\begin{array}{c}\text { Hussein et al. LDV } \\
\text { data (1994) }\end{array}$ \\
\hline $\mathrm{Re}$ & 11,000 & 95,500 & 95,500 \\
\hline $\mathrm{S}$ & 0.096 & 0.102 & 0.094 \\
\hline $\mathrm{B}$ & 5.9 & 5.9 & 5.8 \\
\hline
\end{tabular}

An interesting note about the two relationships mentioned above is that the local Reynolds number, based on the centerline velocity and the jet width, is independent of distance (Pope, 2000). This is shown in Equation 2.4 below.

$$
R e_{0}(x)=\frac{r_{1 / 2}(x) U_{0}(x)}{v}=U_{J} B d S
$$




\section{LITERATURE REVIEW}

\subsection{MINIATURE LDV PROBES}

LDV probes are typically much larger than the probe presented in this thesis. These conventional probes are non-intrusive to the flow but require a window to see the flow which may not always be available. A miniature three-velocity-component LDV probe was developed by Byun et al. (2004) for use where larger probes were unusable. The probe's overall dimensions were 23.3-mm X 8.7-mm X 90.6-mm (.917i-n X .343-in X 3.567-in). A twovelocity-component probe was developed by Esirgemez and Olcmen (2005) for use in internal combustion engines (ICE). The probe was designed to fit into a spark plug hole on an ICE to measure turbulent flow velocities inside the engine cylinder. Another spark plug LDV probe was developed by Ivanchenko et al. (2007) to measure three velocity components as opposed to just two. The probe head had a diameter of $12 \mathrm{~mm}(.472 \mathrm{in})$ and an overall length of $75 \mathrm{~mm}$ (2.953in). The LDV probe head presented in this thesis is much smaller than these probe heads. The probe head of the borescopic LDV probe has a $7 \mathrm{~mm}$ (.276in) diameter which is considerably smaller than any of the probes above.

\subsection{PIPE JET FLOW}

Pipe flow is one of the fundamental flows that have been studied by numerous researchers since the beginning of research on fluid mechanics. Much research exists in 
literature on the effects of Reynolds number, temperature, and roughness on the flow inside, at the exit, and outside of pipes. The jet flow was used to measure the velocity profile at the exit of the pipe. The results were then compared to previous studies performed in literature. The following few studies are cited as needed as they pertain to the research undertaken here.

The exit velocity profile of a long pipe jet is non-uniform, and can be described empirically by a power-law (Mi et al., 2001, Munson, 2002). This fact will be used in this thesis to help confirm the validity of the velocity measurements taken with the borescopic LDV probe designed.

The power law velocity profile is given by Equation 3.1 (Munson, 2002).

$$
\frac{u(r)}{U}=\left(\frac{y}{R}\right)^{\frac{1}{n}}=\left(1-\frac{r}{R}\right)^{\frac{1}{n}}
$$

where $u(r)$ is the velocity profile at the pipe exit, $U$ is the centerline velocity at the pipe exit, $r$, is the radial distance from the center of the pipe, $R$ is the radius of the pipe, and $n$ is an exponent that is a function of Reynolds number. Prior research has shown $n=7$ to be applicable to a wide range of pipe flows and is commonly used. The above equation with $n=7$ has come to be known as the "one seventh power law". It, however, cannot be used to calculate the wall shear stress as due to the infinite velocity gradient it gives at the wall (Munson, 2002).

The symbolic relationship between $\bar{u} / U$ and $n$ is shown in Equation 3.2 (Munson, 2002).

$$
\frac{\bar{u}}{U}=\frac{2 n^{2}}{2 n^{2}+3 n+1}
$$

For $n=7$, the value of $\bar{u} / U$ can be calculated to be $0.81 \overline{6}$. 
Toonder and Nieuwstadt (1997) studied the effect of Reynolds number on flow through a pipe, using water as the working fluid. The study showed a clear dependence of the mean velocity profile on the Reynolds number for $\operatorname{Re}<25000$. They presented their results in a nondimensionalized form using the wall variables in a logarithmic scale. Their results also showed the validity of the universal logarithmic law with slope 2.5 and additive constant 5.0 for

$$
R e=\frac{U_{b} D}{v}>25,000
$$

where $\mathrm{U}_{\mathrm{b}}$ is the bulk mean velocity, D is the diameter of the pipe, and $v$ is the kinematic viscosity.

The power law velocity profile, in fully developed turbulent pipe flow inside the pipe, is given by Equation 3.1 (Afzal et al., 2007).

$$
u_{+}=C y_{+}^{\alpha}
$$

where $u_{+}$and $y_{+}$are the non-dimensionalized velocity and distance from the wall of the pipe, respectively, given by Equations 3.2 and 3.3.

$$
\begin{gathered}
u_{+}=\frac{u}{u_{\tau}} \\
y_{+}=\frac{y u_{\tau}}{v}
\end{gathered}
$$

where $u$ is the velocity in the streamwise $\mathrm{x}$-direction, $v$ is the molecular kinematic viscosity, and $\mathrm{u}_{\tau}$ is the friction velocity given by Equation 3.4. 


$$
u_{\tau}=\sqrt{\frac{\tau_{w}}{\rho}}
$$

where $\tau_{\mathrm{w}}$ is the skin friction on the wall and $\rho$ is the density of the fluid.

Numerous studies to experimentally determine the power law index, $\alpha$, and its prefactor, $\mathrm{C}$, have been conducted. The following is a list of the results of some of these studies.

- Blasius 1912: $\alpha=0.143 ; C=8.74$

- Duncan et al. 1967: $\alpha=0.111 ; C=10.6$

- Zagarola and Smits 1998: $\alpha=0.137 ; \mathrm{C}=8.70$

- McKeon et al. 2004: $\alpha=0.142 ; \mathrm{C}=8.49$

\subsection{CONVERGENT NOZZLE JET FLOW}

Nozzles are described as convergent when they narrow from a wide diameter to a smaller diameter. Convergent nozzles are used to accelerate subsonic fluids up to sonic velocity.

Kassab et al. (1996) used laser Doppler anemometry to investigate certain aspects related to the characteristics of turbulent jet flow, including:

- The effect of velocity bias on the experimental results of the mean and fluctuating velocities.

- The effect of upstream conditions on the mean and turbulent velocity profiles at the jet exit plane. 
- The decay rate of the center line longitudinal mean velocity and how it compares to empirical results.

- The achievement of self-preservation of the jet flow.

- Determining whether the flow approaches isotropy or not.

- Documenting of the distribution of the radial mean as well as turbulent velocity profiles.

The study showed that, (i) no correction for the velocity bias is needed for radial velocity results when the turbulence intensity is below $15 \%$, (ii) The mean velocity profiles cannot uniquely identify the state of a jet flow, (iii) The effect of the upstream conditions is pronounced mainly in the near field region and, (iv) The jet flow approaches certain degree of isotropy in the far field.

Boersma et al. (1997) studied the effect of the inflow conditions on the self-similar region of a jet. They used direct numerical simulation for their study. By applying a tractionfree boundary condition at the lateral boundaries, they were able to obtain a realistic solution for the entrainment of a jet that compared well to experimental results obtained from other studies. They compared the results obtained from two separate experiments using two separate initial profiles and determined that their results did not support the hypothesis of universal selfsimilarity. However, when using a different scaling proposed by George (1989), the agreement between both simulations became much better, supporting George's conclusion that the difference between data obtained in various jet experiments found in literature may not be solely due to experimental errors but may also be attributed to improper scaling. George showed that the equations of motion allow similarity solutions which can still depend on factors such as the initial conditions. George's analysis was possible only if 


$$
R_{S} \propto U_{S}^{2} \frac{\partial \delta}{\partial z}
$$

where $R_{S}$ is a scale for the Reynolds stress and $U_{S}$ is a velocity scale. This is opposed to the traditional $R_{S}=U_{S}^{2}$

Ferdman et al. (1999) studied the effects of non-uniform initial velocity profiles on the downstream evolution of round turbulent incompressible jets experimentally using hot-wire anemometry. Jets evolving from two non-uniform initial velocities profiles, one with an axisymmetric fully developed profile and the other with an asymmetric initial profile, were compared to a jet with top-hat initial velocity distribution. The Reynolds number of both jets was $2.4 * 10^{4}$. The study showed that jets with fully shearing (where the shear layer is the full width of the jet with a high level of turbulence) initial mean velocity profiles tend to have smaller far-field mixing and growth rates than the top-hat profile jets generated with a convergent nozzle. The study also showed that the far-field behavior of jets with non-uniform initial velocity profiles are far less sensitive to differences in initial turbulence intensity levels and velocity profile shapes.

The designed borescopic probe was tested in a top-hat profile jet flow generated using a hot-wire calibrator low seeded with D-Octyle Phthalate particles. This flow setup was previously used by Esirgemez and Olcmen (2007). The centerline velocity was measured up to about fifty diameters away from the exit of the jet and the results compared to other studies mentioned above on jet centerline velocity decay. 


\section{LASER DOPPLER VELOCIMETRY TECHNIQUE}

This chapter focuses on the LDV technique and the components of an LDV system. The technique is described using its historical background and its working principles. An overview of the signal processing and data reduction techniques is then discussed.

\subsection{LASER}

Laser is actually an acronym for Light Amplification by Stimulated Emission of Radiation. Lasers date back to the early 1960s. Light, emitted from atoms, stimulates the emission of more light from other atoms to generate a laser beam (Watrasiewicz, 1976). Light emitted from lasers is very intense, coherent, directional, and monochromatic (Encarta, 2000). In other words, laser beams are extremely bright and are pure in color and frequency.

Lasers are classified depending on the medium used. The most common classifications are solid state, liquid, gas, and semiconductor (Encarta, 2000). Solid state lasers use different types of crystals as media and generate the highest power output. They range in frequency from infrared to ultraviolet and X-ray frequencies can be achieved using yttrium. Glass vessels containing inorganic dyes are the most common media used by liquid lasers. The lasers are created using intense pulsing flash lamps or by gas lasers operating in continuous wave mode (Encarta, 2000). 
The most compact lasers are semiconductor lasers. Gallium arsenide is the most commonly used semiconductor. Semiconductor lasers work by passing an electrical current across a junction between layers of semiconductors with differing conducting properties which energizes the lasers and allows them to operate in continuous wave mode with efficiency greater than 50 percent.

Gas lasers, like the argon-ion laser used for this probe, use different gasses as media. The gases are usually contained in tubes made of glass or quartz. The lasers are excited using chemical reactions, electron beams, electrical currents, or ultraviolet light.

Lasers are used in many different fields. Lasers are used in the medical field for various types of surgery for precision cutting and welding. Industry uses the fact that lasers can be focused on a tiny area with large amounts of power for cutting and drilling. The military uses lasers for weapons and guidance systems. Scientific researchers in different fields utilize the highly directional and monochromatic properties of lasers to measure small frequency shifts that occur when light scatters off an object. Communication satellites use the fact that laser beams can travel long distances without losing their power. Artists can even use lasers for engraving or creating extravagant light shows.

\subsection{HISTORICAL BACKGROUND}

With the invention of the laser in the early 1960s, researchers began to consider using the Doppler Effect and lasers to measure a particle's velocity. The Doppler Effect was discovered and described by nineteenth century Austrian physicist Christian Doppler (Encarta, 2000). The Doppler Effect is based on the frequency change of the light due to the relative motion of the observer and the source. For instance, with a stationary sound source, as the observer moves 
towards the source, the frequency observed increases. As the observer moves away from the sound source, the frequency decreases. When the observer moves towards the source, the source appears to have a higher pitch. When the observer moves away from the source, the source appears to have a lower pitch. Albert Einstein described a similar Doppler Effect occurring with light waves in his special theory of relativity (Encarta, 2000).

The velocity of a particle as it scatters light can be measured using the Doppler Effect. The scattered light's frequency changes with the movement of the particle. The frequency change is proportional to $2 V_{p} / c$, where $V_{p}$ is the particle's velocity and $c$ is the speed of light. The frequency shift is not easily detected with typical spectroscopic techniques due to the speed of light being so much larger than the velocity of the particle.

In 1964, Yeh and Cummins became the first researchers to demonstrate that the velocity of a fluid could be measured using the Doppler Effect. They employed the technique to measure the velocity of laminar water flow. With $0.557 \mu \mathrm{m}$ diameter polystyrene spheres for seeding, they used a helium-neon laser and red light at a wavelength $632.8 \mathrm{~nm}$ to measure the frequency shift of the scattered light (Yeh and Cummins, 1964). Their experimental setup is shown in Figure 4.1. 


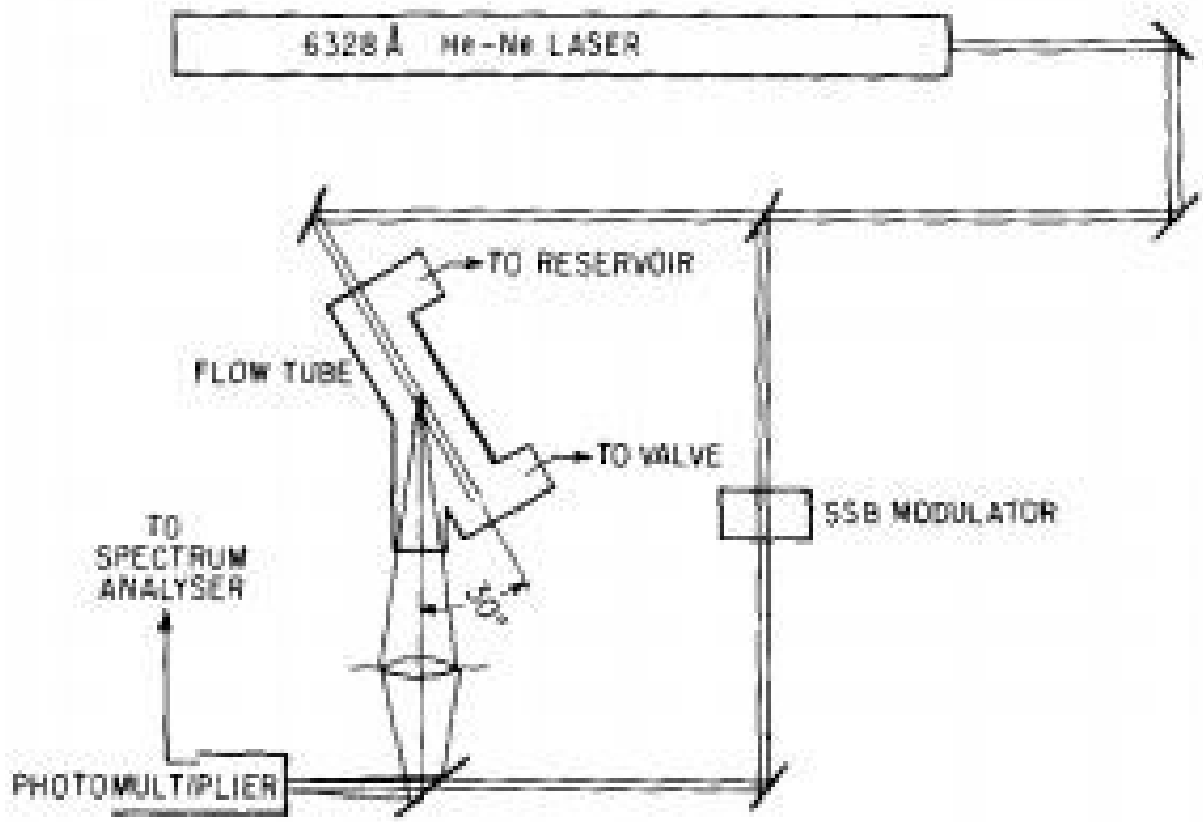

Figure 4.1 First LDV setup used (Yeh and Cummins, 1964).

As seen in Figure 4.1, Yeh and Cummins used a beam splitter to split the laser beam into two beams using two mirrors to turn the beam $180^{\circ}$. The signal beam bounces off a third mirror and is directed into the flow tube. The reference beam is passed through a modulator that shifts the frequency of the beam by $30 \mathrm{MHz}$. The two beams are then remixed and directed toward the photomultiplier tube by another mirror/splitter plate. The photomultiplier outputs a high frequency and time varying signal superimposed on a low frequency signal. The high frequency content is measured using a spectrum analyzer. A $30 \mathrm{MHz}$ frequency indicates zero velocity since it matches the shift frequency of the reference beam. A frequency different from $30 \mathrm{MHz}$ indicates a velocity different from zero and the shift is added to the $30 \mathrm{MHz}$ (Watrasiewicz, 1976). 


\subsection{LDV - WORKING PRINCIPLE}

Basic information about the flow velocity range is needed to correctly set up an LDV system. The measurement volume dimensions and concentration of seeding particles are both determined based on this information. With this information, components of the LDV system can be chosen and the system can be designed (Albrecht et al., 2003).

Monochromatic laser light is used as a light source in the LDV technique. Two mutually coherent laser beams intersect with each other and interfere constructively and destructively to form fringes. The intersecting region is known as the probe or measurement volume. The velocity of the flow is measured as seeding particles in the flow pass through the measurement volume which can be seen in Figure 4.2. For this reason, the LDV technique is known as an indirect measurement technique. It is assumed that the velocity of the particle represents the velocity of the flow as long as the particle is not lagging the flow. If this happens, the lag needs to be calculated and taken into account, limiting the useful data range (Albrecth et al. 2003). The basic working principle of the LDV technique is shown in Figure 4.3.

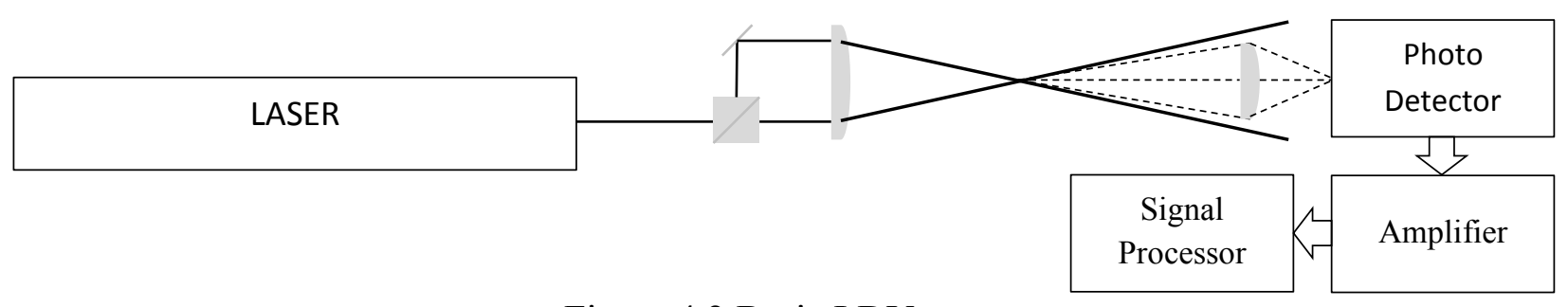

Figure 4.2 Basic LDV setup 


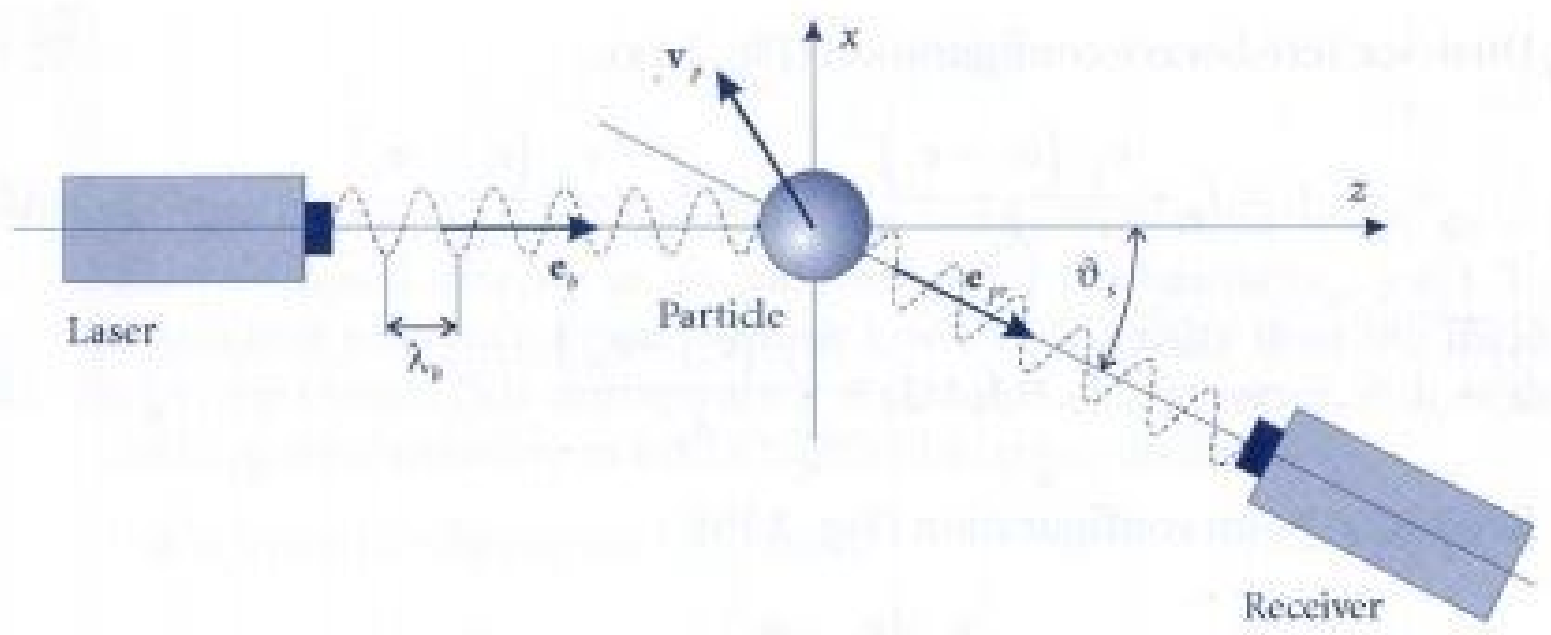

Figure 4.3 Working principle of LDV technique (Albrecht et al., 2003).

Equation 4.1 shows the relationship between the frequency of light scattered from a particle and the frequency received by a detector (Albrecht et al., 2003).

$$
\begin{gathered}
f_{r}=f_{p} \frac{1}{1-\frac{\bar{e}_{p r * \vec{v}_{p}}}{c}}=f_{b} \frac{1-\frac{\vec{e}_{b} * \vec{v}_{p}}{c}}{1-\frac{\bar{e}_{p r *} * \vec{v}_{p}}{c}} \approx f_{b}+f_{b} \frac{\vec{v}_{p} *\left(\vec{e}_{p r}-\vec{e}_{b}\right)}{c}=f_{b}+\frac{\vec{v}_{p} *\left(\vec{e}_{p r}-\vec{e}_{b}\right)}{\lambda} \\
\left(\left|\vec{v}_{p}\right| \ll c\right), c=f_{b} \lambda
\end{gathered}
$$

The range of the Doppler shift is usually from several kilohertz to a couple hundred megahertz which is several orders of magnitude smaller than the $10^{14} \mathrm{~Hz}$ frequency of the laser light. Because this shift is so small, it is not easily detected. Several pieces of electronic equipment are used to detect the shift. Photon correlators, counters, and frequency analyzers are just some of the types of equipment used to detect the shift. 
The Doppler shift frequency can be calculated by adding the frequency of the two beams. The resulting equation can then be simplified to yield Equation 4.2.

$$
f_{D}=\frac{2 \sin \left(\frac{\Theta}{2}\right)}{\lambda}\left|V_{p}\right| \cos (\alpha)=\frac{2 \sin \left(\frac{\Theta}{2}\right)}{\lambda} V_{p \perp} \quad\left(V_{p \perp}=\left|V_{p}\right| \cos (\alpha)\right)
$$

The angles, $\Theta$ and $\alpha$, and the velocity, $V_{p}$ are shown in Figure 4.4 below.

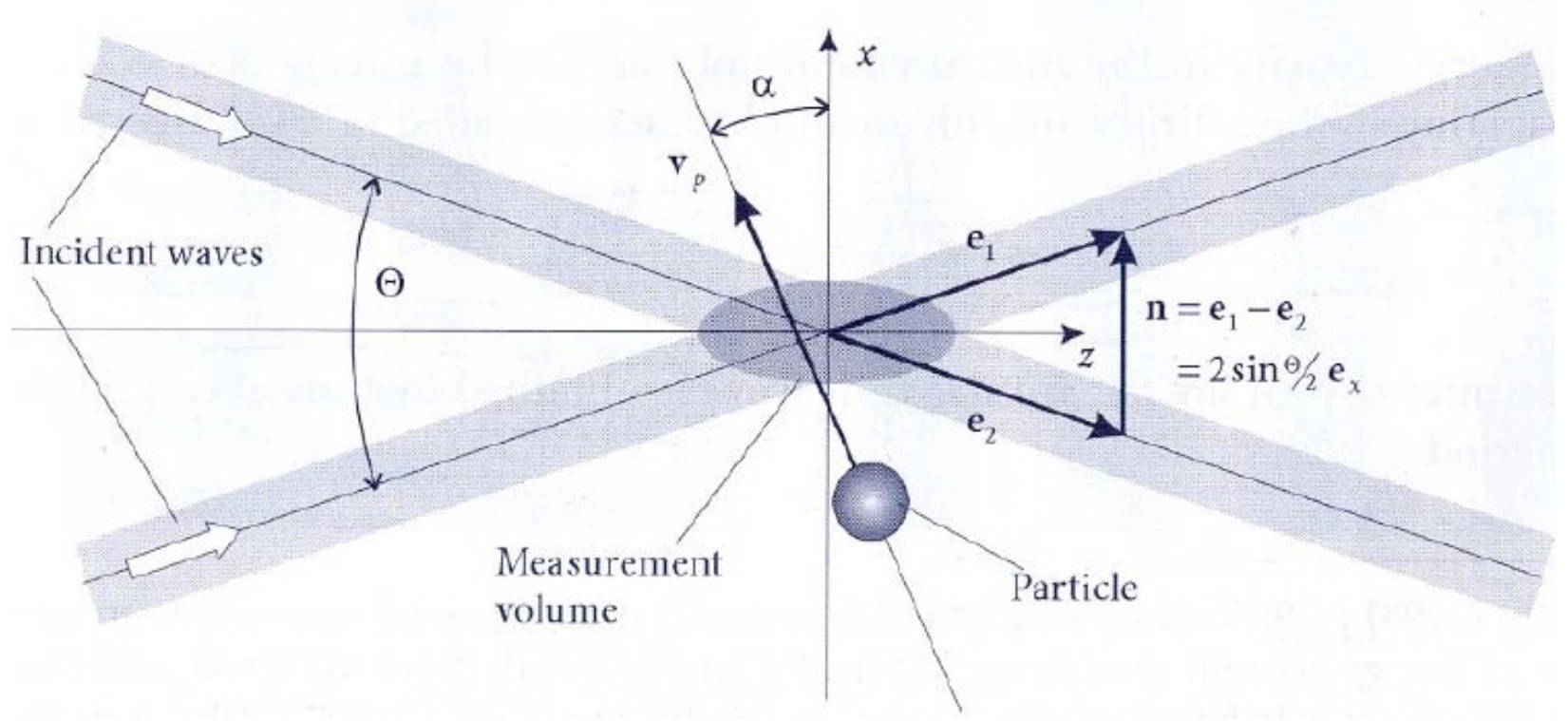

Figure 4.4 LDV - Intersecting beams and vector relations (Albrecht et al., 2003).

The fringe spacing in the measurement volume depends on the laser wavelength and the angle created between the intersecting beams. Figure 4.5 shows the fringes and defines the fringe space. Equation 4.3 gives the value of the fringe space $\Delta x$. 


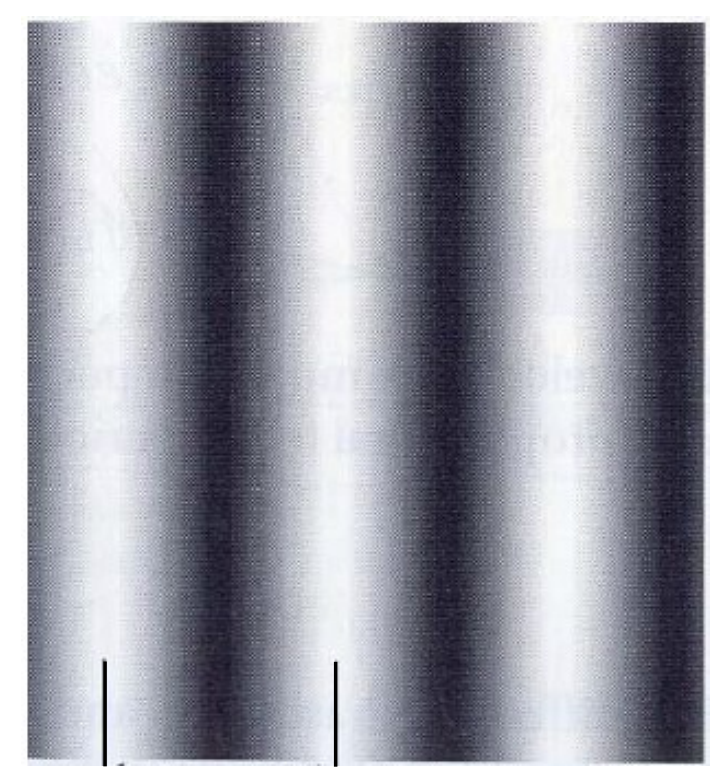

Figure 4.5 LDV - Fringe pattern and spacing (Albrecht et al., 2003).

$$
\Delta x=\frac{\lambda}{2 \sin \left(\frac{\Theta}{2}\right)}
$$

The velocity component perpendicular to the fringes, $V_{p \perp}$, is inversely proportional to the period of the fringe crossing $\mathrm{T}_{\mathrm{D}}$. This is shown in Equation 4.4.

$$
V_{p \perp}=\frac{\Delta x}{T_{D}}
$$

The frequency signal is acquired when particles pass through the measurement volume. This signal, however, does not contain any information about the direction the particles are moving. Consequently, particles moving at the same speed in the opposite direction would give the same frequency. This issue is overcome using a frequency shifting technique shown in Figure 4.6. Acousto-optic modulators are used to shift the wavelength of one of the laser beams forming the fringes. The fringes move with constant velocity due to the frequency shift. If the particle is moving in the same direction as the fringes, the collected light's frequency is equal to 
the difference between the Doppler and shifting frequency. Conversely, if the particle is moving in the opposite direction of the fringes, the collected light's frequency is equal to the sum of the Doppler and shifting frequency. The collected light frequency from a stationary particle is equal to the modulator shift frequency. Equation 4.5 shows this relation.

$$
f_{r}=f_{s h}+\frac{\vec{V}_{p}\left(\vec{e}_{1}-\vec{e}_{2}\right)}{\lambda}=f_{s h} \pm \frac{2 \sin \left(\frac{\Theta}{2}\right)}{\lambda}\left|\vec{V}_{p \perp}\right|=f_{s h} \pm f_{D}
$$
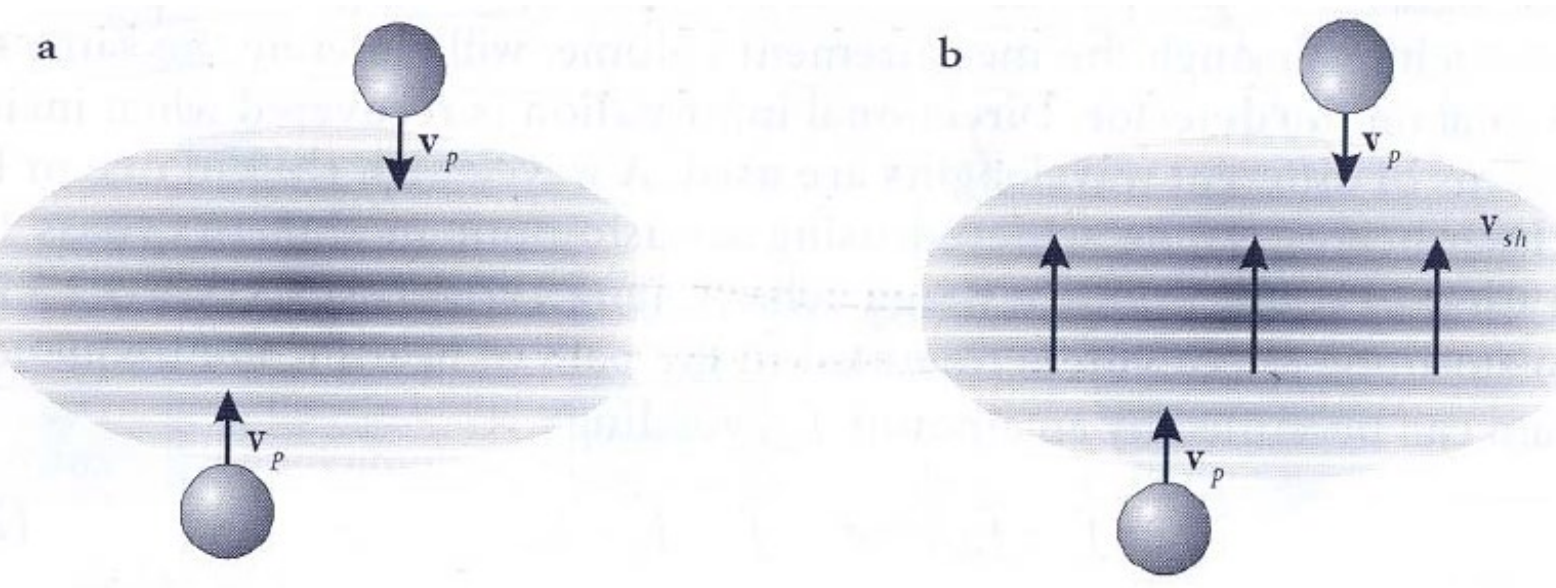

Figure 4.6 LDV - Particle movement in fringe pattern (Albrecht et al., 2003).

a) without frequency shift. b) with frequency shift.

\subsection{MEASUREMENT VOLUME}

The measurement volume where the interference fringes are parallel to each other is generated by overlapping two laser beams at their respective waist positions. If this is not done, the fringe pattern at the measurement volume can be distorted as seen in Figure 4.7. Misalignment of optics and improper design of the transmitting system are the typical causes of this type of problem. 
a Longitudinal distortion

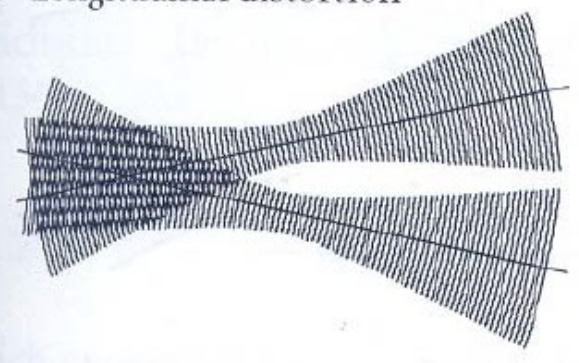

b Transverse distortion

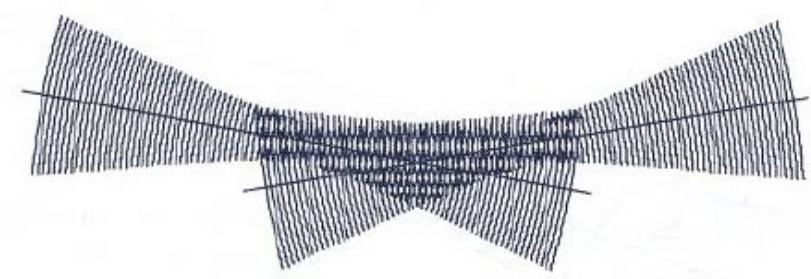

Figure 4.7 Distortion of fringes at measurement volume (Albrecht et al., 2003).

The measurement volume is the three dimensional ellipsoid that forms where two laser beams intersect each other. Figure 4.8 shows an expanded view of a measurement volume and its dimensions.

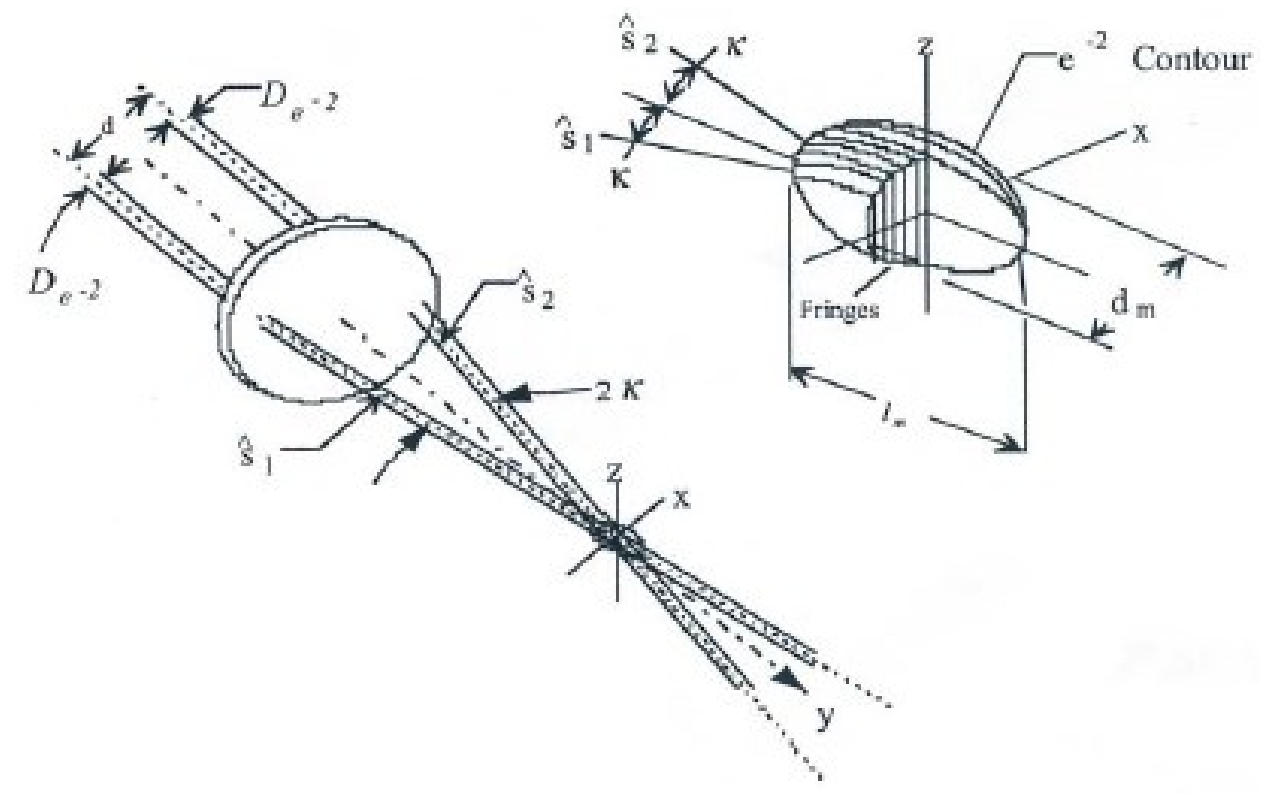

Figure 4.8 LDV - Measurement volume (Albrecht et al., 2003). 
The following equations are used to find the dimensions and other properties of the measurement volume.

$$
\begin{gathered}
N_{f r}=\frac{d_{m}}{d_{f}}=\frac{1.27 d}{D_{e^{-2}}} \\
d_{m}=\frac{4 f \lambda}{\pi D_{e^{-2}} \cos (\kappa)} \\
l_{m}=\frac{4 f \lambda}{\pi D_{e^{-2}} \sin (\kappa)} \\
V=\frac{\pi\left(\frac{4 f \lambda}{\pi D}\right)}{6 \cos ^{-2}(\kappa) \sin (\kappa)}
\end{gathered}
$$

\subsection{SIGNAL EXTRACTION}

The measurement of velocity components and turbulence parameters depends on the measurement of the frequency of the signal obtained when a particle passes through the measurement volume (Albrecht et al., 2003). The signal's frequency is referred to as the Doppler frequency given by Equation 4.2. In the laser Doppler signal processing, both the fringe spacing and the velocity are assumed to be constant throughout the measurement volume (Albrecht et al., 2003).

Figure 4.9(a) shows the original signal as it is detected from the photomultiplier and the Discrete Fourier Transform (See Appendix B for more information on Discrete Fourier Transforms) of the signal in the frequency domain. The figure shows how the signal envelope assumes a Gaussian shape following the Gaussian intensity distribution within the cross section 
of the measurement volume (Albrecht et al., 2003). The disturbing frequency shown in the figure is undesirable as it affects the calculation of the Doppler signal. This disturbing frequency is eliminated using a low-pass filter to filter out the undesired frequency. Figure 4.9(b) shows the signal and spectrum after the signal passes through the filter. The Doppler frequency is found using the peak of the signal frequency in the low-pass filtered spectrum. Once the Doppler frequency is determined, the flow velocities can be calculated using Equation 4.2.
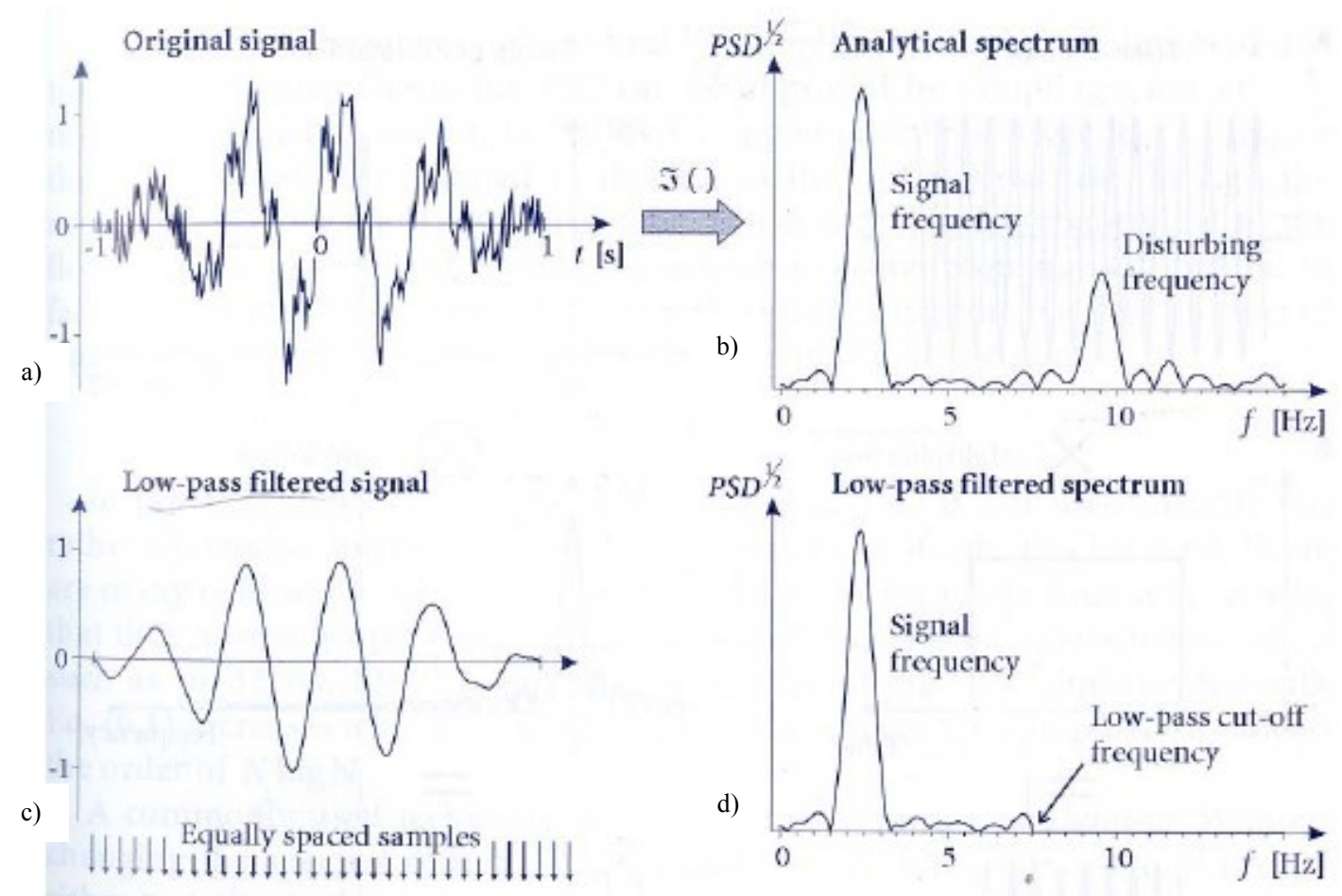

Figure 4.9 Detected signal and its spectrum (Albrecht et al., 2003).

a) Original signal and spectrum. b) Low-pass filtered signal and spectrum.

Another source of error in the signal is noise generated by the unwanted light collected from sources other than the particle and by the electronic devices used for signal processing. The noise results in higher spectral values in the frequency domain. If the noise is too great, it may result in inaccurate Doppler frequency values. Signal-to-noise ratio, SNR, is a measure of 
the level of noise. Equation 4.10 shows how the SNR per decibel of a signal is calculated; it is the ratio of the power of signal fluctuations to the power of noise fluctuations.

$$
\frac{S N R}{d B}=+10 \log \left|\frac{\sigma_{s}^{2}}{\sigma_{n}^{2}}\right|
$$

where $\sigma_{\mathrm{s}}$ is the signal fluctuations and $\sigma_{\mathrm{n}}$ is the noise fluctuations.

\subsection{TURBULENCE PARAMETERS AND MEASUREMENT}

Some of the turbulence parameters than can be calculated using the instantaneous velocity as measured by an LDV system are listed below (Watrasiewicz, 1976):

- Turbulent Fluctuation Velocity: Difference between the instantaneous velocity and the mean velocity.

- Reynolds Stresses: Organized motions result in velocity fluctuations which act as shear stresses in the flow field. Correlations obtained between the fluctuating velocities multiplied with density are examples of such stresses.

- Turbulent Kinetic Energy: Energy that drives turbulence. $T K E=\frac{1}{2}\left(\bar{u}^{2}+\bar{v}^{2}+\bar{w}^{2}\right)$

- Turbulent Energy Spectrum Density: The turbulence energy with wave numbers between $k$ and $k+d k . k=2 \pi / \lambda$

- Integral Length Scale of Turbulence: Mean size of the energy containing eddies. 
Equations used to calculate turbulent velocities and Reynolds stresses will now be discussed. Explanations of the other turbulence parameters can be found in (Watrasiewicz, 1976).

Reynolds averaging technique is used to calculate the normal and shear stresses. For this technique, the instantaneous velocity is first expressed as the sum of the mean velocity and velocity fluctuations around the mean value.

Given that the instantaneous velocity component perpendicular to the fringes within the measurement volume is $U$, the mean velocity is $\bar{U}$, and the fluctuating velocity is $u$ :

$$
U=\bar{U}+u
$$

Squaring both sides of Equation 4.11,

$$
\begin{aligned}
& U^{2}=\{(\bar{U}+u)(\bar{U}+u)\} \\
& U^{2}=\left\{\bar{U}^{2}+2 u \bar{U}+u^{2}\right\}
\end{aligned}
$$

The fluctuating velocity is defined as the variation of the velocity component from the mean velocity in time. The average of value of $u$ is zero and therefore, the second term in Equation 4.13 is zero. Solving Equation 4.13 for $u^{2}$ after dropping the second term yields Equation 4.14.

$$
u^{2}=U^{2}-\bar{U}^{2}
$$




\subsection{PARTICLE SEEDING}

Velocity measurements using the LDV technique require the presence of particles to produce enough scattered light for analysis. In some LDV applications such as water flow, particles that naturally occur in the fluid may be all the seeding that is needed. For most applications, however, seeding is required. Some of the basic requirements expected from seeding particles are summarized below (Menon, 1982):

- Particles should follow the flow speed closely (must be small enough).

- Particles must scatter light sufficiently to produce measurable signals (must be large enough).

- Particles must be present in the flow in desirable amounts.

Some other factors that influence the choice of seeding particles include:

- Parameters of the experiment.

- Magnitude of the velocity fluctuations.

- Light collection angle.

- Fringe spacing.

- Extreme temperatures.

- Reactivity of the fluid.

Particle generation can be divided according to solid and liquid particles. Solid particles or powders are used to seed liquid flows or in applications such as combustion, where liquid droplets would evaporate (Albrecht et al., 2003). Selecting the proper seeding material can significantly improve the performance and accuracy of an LDV system. The most important property when choosing the right seeding particle is its size. It should be small enough to closely 
follow the flow of the fluid but should also be large enough to scatter a sufficient amount of light in order to obtain measurable signals. Signal quality is greatly affected by the geometric and physical properties of the particles (Menon, 1982).

The ability of a particle to follow the flow is related to its aerodynamic diameter (Menon and Lai, 1991). The aerodynamic diameter of an irregularly shaped particle is the diameter of a sphere of unit density that has aerodynamic behavior identical to that of the particle in question. The aerodynamic diameter, $\mathrm{d}_{\mathrm{a}}$, is defined mathematically by Equation 4.15 .

$$
d_{a}=\sqrt{\rho_{p} \frac{C_{d}}{C_{d a}}(a)}
$$

where $\rho_{p}$ is the density, $c_{d}$ and $c_{d a}$ are the Cunningham slip correction factors (See appendix C for more information on the Cunningham slip correction factor) for the seeding particle and idealized particle respectively, and $a$ is the diameter of the particle.

For spherical particles, the aerodynamic diameter is simplified to Equation 4.16.

$$
d_{a}=a \sqrt{\rho_{p}}
$$

In addition to the aerodynamic diameter, two other important factors relate to a particle's ability to follow the flow. One is the ability of the particle to follow rapid changes in velocity and the other pertains to the particle's ability to follow low velocity flows. The first factor is associated with the particles velocity after a step change in flow velocity and the other is associated with the particles settling velocity. 
The instantaneous velocity of a particle in a flow after a step change in fluid velocity is given by Equation 4.17 .

$$
e^{-t / \tau}=\frac{V_{g}-V_{p}}{V_{g i}-V_{p i}}
$$

where $t$ is time, $V_{g}$ is the fluid velocity after step change, $V_{p}$ is the particle velocity at time $t, V_{g i}$ and $V_{p \mathrm{i}}$ are the gas and particle velocity before the step change respectively, and $\tau$ is the relaxation time of the particle given by Equation 4.18 .

$$
\tau=\frac{\rho_{p} a^{2}}{18 \eta}
$$

where $\rho_{p}$ is the density of the particle, $a$ is the diameter of the particle, and $\eta$ is the relative refractive index.

The frequency response for a particle is given by Equation 4.19.

$$
f=\frac{1}{2 \pi \tau}\left[\frac{1}{A_{r}^{2}}-1\right]^{\frac{1}{2}}
$$

where $A_{r}$ is the particle oscillation amplitude divided by the fluid oscillation amplitude.

From Equations 4.15-4.19 it is clear that a particle with a small density and size give the desired small relaxation time and high frequency response. Table 4.1 shows the relaxation time and frequency response at $3 \mathrm{~dB}$ of some common seeding particles. 
Table 4.1 Frequency Response for Particles in Air Flow (Menon and Lai, 1991).

\begin{tabular}{|c|c|c|c|c|c|c|c|c|}
\hline \multicolumn{4}{|c|}{ Diameter } & \multicolumn{2}{c|}{$0.5 \mu \mathrm{m}$} & \multicolumn{2}{c|}{$1.0 \mu \mathrm{m}$} & \multicolumn{2}{c|}{$2.0 \mu \mathrm{m}$} \\
\hline Particles & $\eta$ & $\mathrm{P}_{0}$ & $\tau$ & $\mathrm{f}_{3 \mathrm{~dB}}$ & $\tau$ & $\mathrm{f}_{3 \mathrm{~dB}}$ & $\tau$ & $\mathrm{f}_{3 \mathrm{~dB}}$ \\
\hline Silicon Carbide & 2.60 & 3.30 & 2.55 & 62 & 10.2 & 16 & 41 & 3.9 \\
\hline Alumina & 1.76 & 3.80 & 2.93 & 54 & 11.7 & 14 & 47 & 3.0 \\
\hline Polystyrene & 1.60 & 1.05 & 0.81 & 196 & 3.24 & 52 & 13 & 13 \\
\hline Peanut Oil & 1.47 & 0.91 & 0.70 & 227 & 2.81 & 57 & 11.2 & 14 \\
\hline Microballons & 1.50 & 0.23 & 0.18 & 897 & 0.71 & 224 & 2.84 & 56 \\
\hline
\end{tabular}

$\eta$ : refractive index

$\mathrm{P}_{0}$ : relative density

$\tau$ : relaxation time $(\mathrm{ms})$

$\mathrm{f}_{3 \mathrm{~dB}}$ : frequency response in $\mathrm{KHz}(3 \mathrm{~dB})$

Equation 4.20 gives the settling velocity of a particle, which depends on the density difference between the fluid and the particle and the dynamic viscosity of the fluid.

$$
V_{p s}=\frac{\Delta \rho a^{2} g_{c}}{\mu_{g}}
$$

where $\Delta \rho$ is the density difference, $g_{c}$ is the gravitational acceleration, and $\mu_{g}$ is the dynamic viscosity of the fluid.

Table 4.2 shows the settling times in water of some of the particles mentioned in Table 4.1.

Table 4.2 Settling Velocity of Particles in Water Flow (Menon and Lai, 1991).

\begin{tabular}{|c|c|c|c|c|c|c|}
\hline \multicolumn{7}{|c|}{ Settling Velocity $(\mu \mathrm{m} / \mathrm{s})$} \\
\hline Particles & $\eta_{\mathrm{R}}$ & $\rho_{0}$ & $0.5 \mu \mathrm{m}$ & $1.0 \mu \mathrm{m}$ & $2.0 \mu \mathrm{m}$ & $5.0 \mu \mathrm{m}$ \\
\hline Silicon Carbide & 1.95 & 3.3 & 0.31 & 1.20 & 5.0 & 30.0 \\
\hline Alumina & 1.32 & 3.8 & 0.38 & 1.40 & 6.0 & 38.0 \\
\hline Polystyrene & 1.20 & 1.6 & 0.0007 & 0.027 & 0.11 & 0.68 \\
\hline
\end{tabular}

$\eta_{R}$ : relative refraction index $\rho_{0}$ : relative density 
Another factor that affects a particle's ability to follow a flow closely is the drag force acting on the particle. The drag force can drastically affect velocity measurements because the particle would lag the flow resulting in an error in mean velocity measurements and turbulent flow measurements. One way to overcome the lag is using correction scheme such as those suggested in literature (Menon and Lai, 1991).

Obtaining high quality signals from particles requires that the amount of light scattered from the particles be great enough to produce measurable signals. Smaller particles tend to add more noise than signal and larger particles do not follow the flow as well. For this reason, particles with nearly uniform size are desirable.

The number of particles put into a flow is another important part of obtaining measurable signals. The type of signal analysis is important for choosing a concentration of particles. Letting $N_{p}$ be the number of particles in the flow and $V$ be the measurement volume, the probability of finding a single particle in the measurement volume is greatest when

$$
N_{p} V=1
$$

If more than a single particle is desired, then $\mathrm{N}_{\mathrm{p}}$ should be chosen such that

$$
N_{p} V \gg 1
$$

If more than a single particle is undesirable, then $\mathrm{N}_{\mathrm{p}}$ should be chosen such that

$$
N_{p} V \ll 1
$$

The concentration of particles in the flow is given by Equation 4.23.

$$
N_{p}=\frac{Q_{S} N_{S}}{Q_{T}}
$$


where $Q_{s}$ is the volumetric aerosol output flow rate of the seeder, $N_{s}$ is the particle concentration of the seeder, and $Q_{T}$ is the total volumetric flow rate.

The important factors to consider when choosing particles have been discussed so far. Particle generation techniques to provide the desired particle concentration and size are now considered. Atomization, evaporation/condensation, and dispersion of solid particles are the most common particle generation methods used.

Atomization is a simple technique widely used due to the many reliable and inexpensive atomizers that are commercially available. Atomizers are used to generate liquid droplets using compressed air and can also be used to create solid particles using solutions such as sugar or salt in water. A solution's concentration is the biggest factor in determining the size of the solid particles. The biggest drawback of the atomization process is that the particles usually are not uniform in size. A conventional liquid/compressed air atomizer is shown in Figure 4.10.

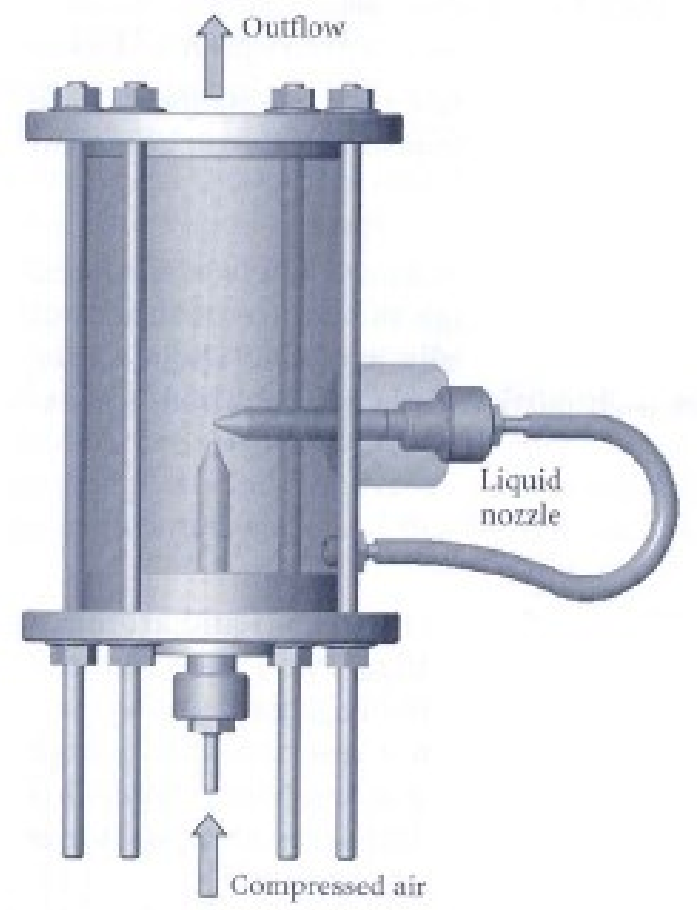

Figure 4.10 Conventional atomizer (Albrecht et al., 2003). 
The evaporation/condensation technique is based on the principal of evaporating a liquid and allowing the vapor to condense. This method varies from atomization in that the generated particles are highly uniform in size. The method uses only oils and, therefore, is not useful for high-temperature applications. Controlling both the oil vapor feed rate and nuclei feed rate is difficult in this method. To solve this problem, an atomizer is first used to atomize the oil droplets before evaporation. A commercially available example of this type of generator is shown in Figure 4.11.

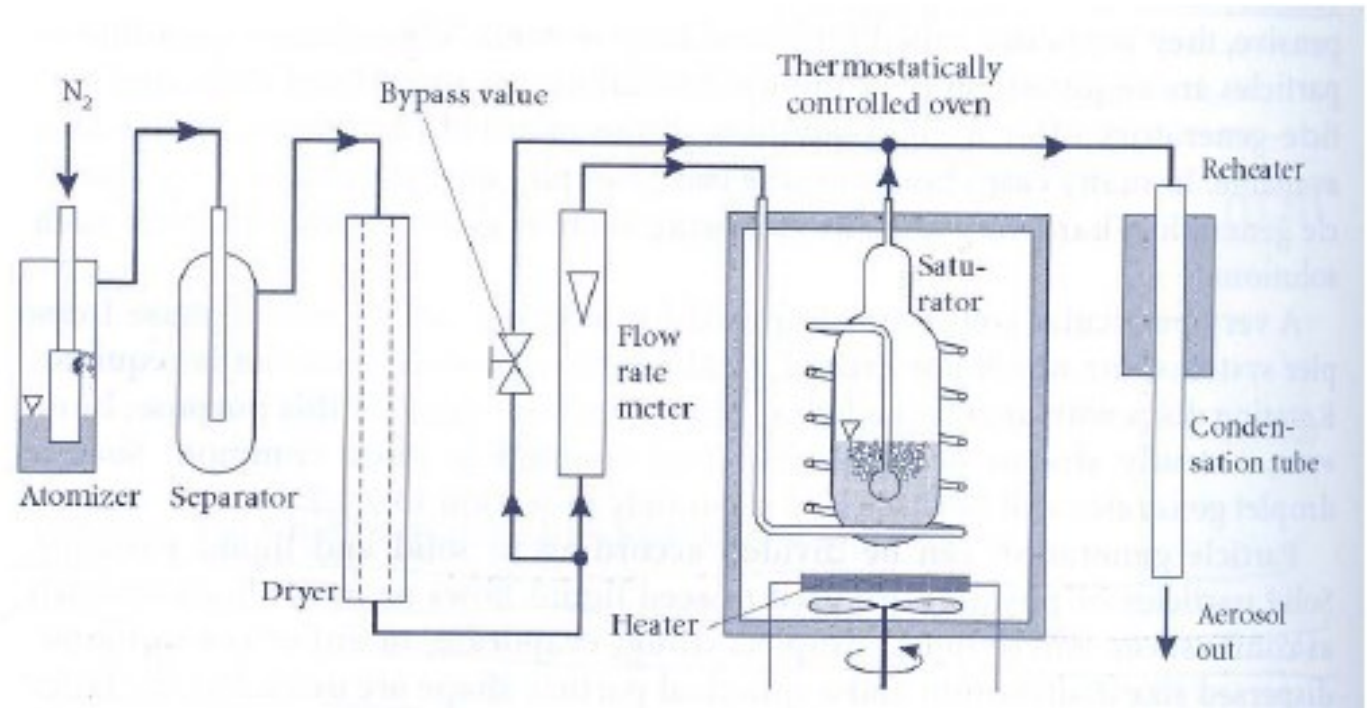

Figure 4.11 Modified Sinclair-La aerosol generator (Albrecht et al., 2003).

Another technique used to generate liquid droplets involves using a vibrating orifice generator. The droplets are generated by applying a periodic disturbance at the appropriate frequency to a liquid jet. The periodic disturbance breaks the jet up into droplets that are highly uniform in size.

The last particle generating method to be discussed is solid particle dispersion. This method, however, is difficult in application. Breaking the material to the desired size and feeding the particles at a constant rate are where the difficulties arise. A solution to these 
problems is using a fluidized bed aerosol generator. Brass beads in the generator are kept floating by the drag force of the air. The solid particles collide with the brass beads and break apart to form the seeding particles. This aerosol generator provides a high flow rate and concentration. Particles for high temperature LDV applications can be generated using this type of seeding generator.

Experimental conditions and desired particle size are the biggest factors in the selection of particle generation method. Many practical generators and particle materials to be used with these generators are commercially available. More information about particle generation techniques can be found in (Albrecht et al., 2003; Menon and Lai, 1991). 


\section{PROBE DESIGN}

\subsection{PRELIMINARY DESIGN}

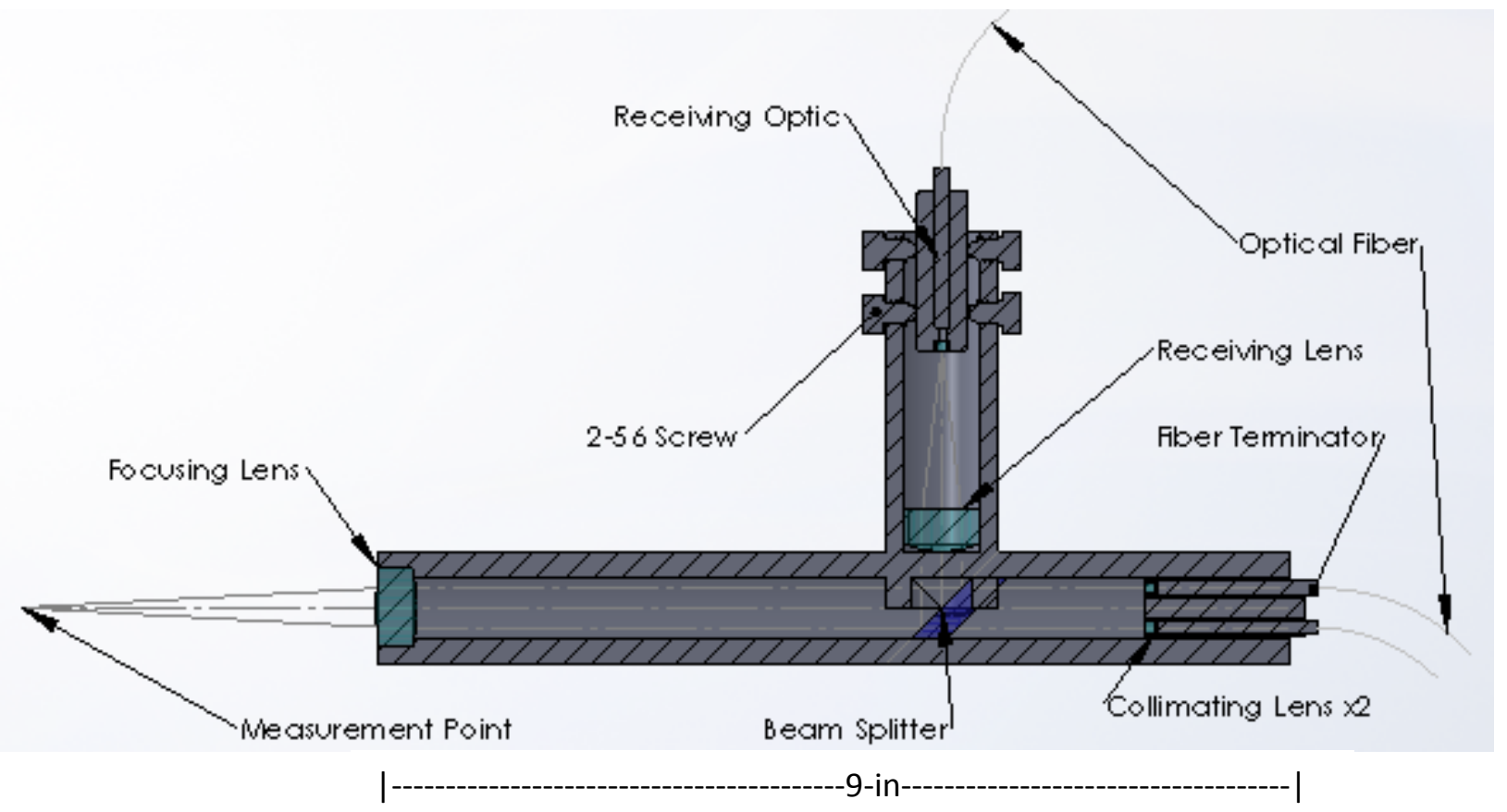

Figure 5.1 Conceptual probe design.

The preliminary design was more of a conceptual idea rather than an actual design. This design is shown in Figure 5.1. As mentioned in section 1.2, the fibers are terminated and inserted into tubes that have collimating lenses attached to the end of them. This original design used 1-mm (.039-in) diameter lenses to collimate the light. The light then passes through a beam splitter that allows the beams to pass through unimpeded to the focusing lens. The beams are crossed at the focal point of the lens and the scattered light collected passes back through the lens 
to the beam splitter that now acts like a mirror that reflects the light $90^{\circ}$ to pass through the receiving lens that focuses the light into the receiving optic fiber.

The design is more conceptual due to the way the transmitting part of the probe and receiving part are connected. Also, a beam splitter plate that acts in the way described, allowing light to pass through from one direction but reflect when coming from the other direction, was not commercially available at the desired size. The final issue came with the 1 -mm (.039-in) collimating lenses. These lenses proved to be impossible to glue into place without getting glue

on the front or back side of the lenses. Also, due to their small size, they were easily lost and extremely hard to handle.

\subsection{FINAL DESIGN}

The first problem solved for the final probe design was how to join the transmitting and receiving ends of the probe together. This was accomplished by machining a block to hold the transmitting, receiving, and probe head tubes seen in Figure 5.1. This block is shown in detail in Figure 5.2. There is a third unseen hole in the figure machined from the back side of the block with a diameter of 0.240 -in $(6.10-\mathrm{mm})$. The probe head long-neck extension tube was glued into the 0.280 -in $(7.11-\mathrm{mm})$ hole, the receiving optic tube was glued into the 0.316 -in $(8.03-\mathrm{mm})$ hole, and the tube holding the transmitting fiber terminator assembly was glued into the 0.240 -in $(6.10-\mathrm{mm})$ unseen hole. The 0.398 -in $(10.11-\mathrm{mm})$ square machined through the side of the probe holds the half cube mirrored prism that acts as the beam splitter plate mentioned in section 3.1. Most of the remaining parts were purchased and are listed in Table 5.2. 


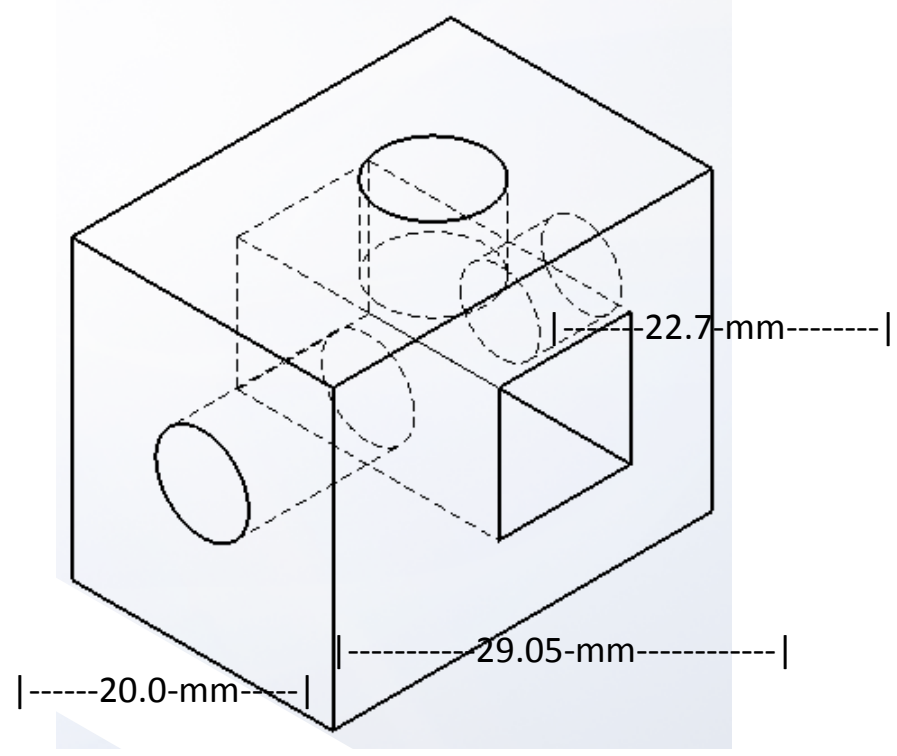

Figure 5.2 Main probe structure block.

Table 5.1 Purchased LDV probe components price list.

\begin{tabular}{|c|c|c|c|c|c|c|c|c|c|}
\hline & Vendor & Part \# & OD[in] & ID[in] & Th[in] & $L[f t]$ & Qty. & Price & Total Price \\
\hline $\begin{array}{c}\text { Probe Head } \\
\text { Tube }\end{array}$ & McMaster-Carr & $50415 \mathrm{~K} 221$ & 0.276 & 0.256 & 0.01 & 3.28 & 1 & $\$ 36.18$ & $\$ 36.18$ \\
\hline $\begin{array}{c}\text { Laser } \\
\text { AssemblyTube }\end{array}$ & McMaster-Carr & $50415 \mathrm{~K} 165$ & 0.236 & 0.197 & 0.02 & 1.64 & 1 & $\$ 14.00$ & $\$ 14.00$ \\
\hline $\begin{array}{l}\text { Receiving } \\
\text { Tube }\end{array}$ & McMaster-Carr & $1800 \mathrm{~T} 311$ & 0.3125 & 0.256 & 0.028 & 1 & 1 & $\$ 20.11$ & $\$ 20.11$ \\
\hline $\begin{array}{c}\text { Terminator } \\
\text { Tube Holder }\end{array}$ & McMaster-Carr & $89935 \mathrm{~K} 225$ & 0.072 & 0.063 & 0.005 & 1 & 1 & $\$ 15.23$ & $\$ 15.23$ \\
\hline \multirow[t]{2}{*}{$\begin{array}{c}\text { Terminator } \\
\text { Tube }\end{array}$} & McMaster-Carr & $51755 \mathrm{~K} 13$ & 0.0625 & 0.042 & 0.01 & 1 & 1 & $\$ 5.02$ & $\$ 5.02$ \\
\hline & Vendor & Part \# & $\mathrm{D}[\mathrm{mm}]$ & FL[mm] & & & Qty. & Price & Total Price \\
\hline Focusing Lens & Edmund Optics & NT45-135 & 6.25 & 30 & & & 1 & $\$ 47.50$ & $\$ 47.50$ \\
\hline $\begin{array}{c}\text { Receiving } \\
\text { Lens }\end{array}$ & Edmund Optics & NT32-301 & 6.25 & 15 & & & 1 & $\$ 47.50$ & $\$ 47.50$ \\
\hline \multirow[t]{2}{*}{$\begin{array}{c}\text { Collimating } \\
\text { Lenses }\end{array}$} & Edmund Optics & NT65-564 & 2 & 1.5 & & & 6 & $\$ 74.50$ & $\$ 447.00$ \\
\hline & Vendor & Part \# & Size $[\mathrm{mm}]$ & WL[nm] & & & Qty. & Price & Total Price \\
\hline $\begin{array}{l}\text { Right Angle } \\
\text { Mirror }\end{array}$ & ThorLabs & MRA10-E02 & 10 & $400-750$ & & & 2 & $\$ 96.80$ & $\$ 193.60$ \\
\hline
\end{tabular}


The last item on the list is the solution to the second problem of the conceptual design. A right angle mirrored prism was purchased and two 0.040-in (1.02- $\mathrm{mm})$ holes were drilled through the prism using diamond coated drill bits to allow the collimated laser beams to pass through unimpeded. Figure 5.3 shows the prism and its dimensions.

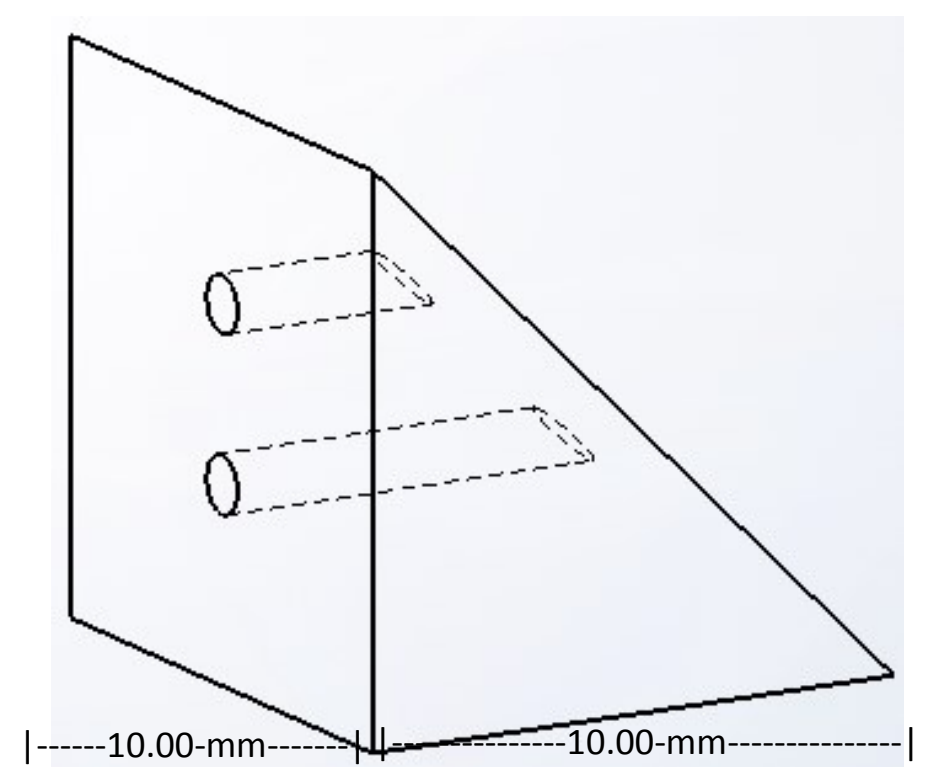

Figure 5.3 Right angle prism dielectric mirror.

The third problem was solved by finding lenses with the same focal length of the 0.039-in (1-mm) lenses but with a 0.079-in (2-mm) diameter. The lenses chosen are listed as collimating lenses in Table 5.1. These lenses were approximately four times bigger in area than the smaller lenses and proved to be much easier to work with. The lenses were glued onto the tubes that the fiber terminator tubes slid into. First, these fiber-terminator holder tubes were centered and glued onto a machined separation plate with a thickness of 0.014-in (0.36-mm) shown in Figure 5.4. The next step was to apply a thin film of slow drying model glue around the rim of each of the fiber-terminator holder tubes and place each lens on the tubes. Next, the fiber-terminators were inserted into the fiber-terminator holder tubes. The lenses were then 
centered by shining the laser beams onto the ceiling and equalizing the distance between the beams on the ceiling and the distance between the terminator tubes. Each lens was then glued permanently into place using cyanoacrylate jewelry glue. Each fiber-terminator holder tube was then inserted into a larger tube to complete the construction of the transmitting fiber-terminators holder assembly. The assembly was then glued into a larger tube which was then glued into the block in Figure 5.2.

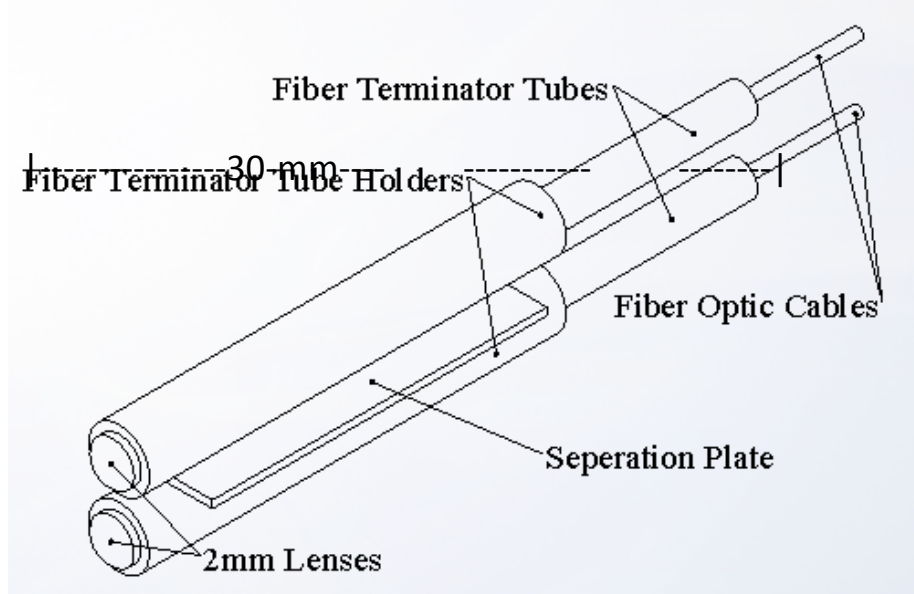

Figure 5.4 Transmitting fiber-terminators holder assembly.

The receiving optics assembly was constructed next. A fiber optic cable was terminated and inserted into a steel structural tube similar to the transmitting fiber-terminators. This tube was then inserted into an octagonal tube machined by the machine shop and held in place with a \#2-56 screw. A block to allow set screws to be used was machined and placed over the tube that joins the receiving optic assembly to the main probe assembly which was then glued into place. The octagonal tube was inserted into a larger tube and held in place with eight \#2-56 set screws to allow movement in three directions. Finally, the receiving lens from Table 5.1 was glued into the bottom end of the tube. The receiving optic assembly is shown in Figure 5.5. The assembly was inserted and glued into the top of the block in Figure 5.2. 


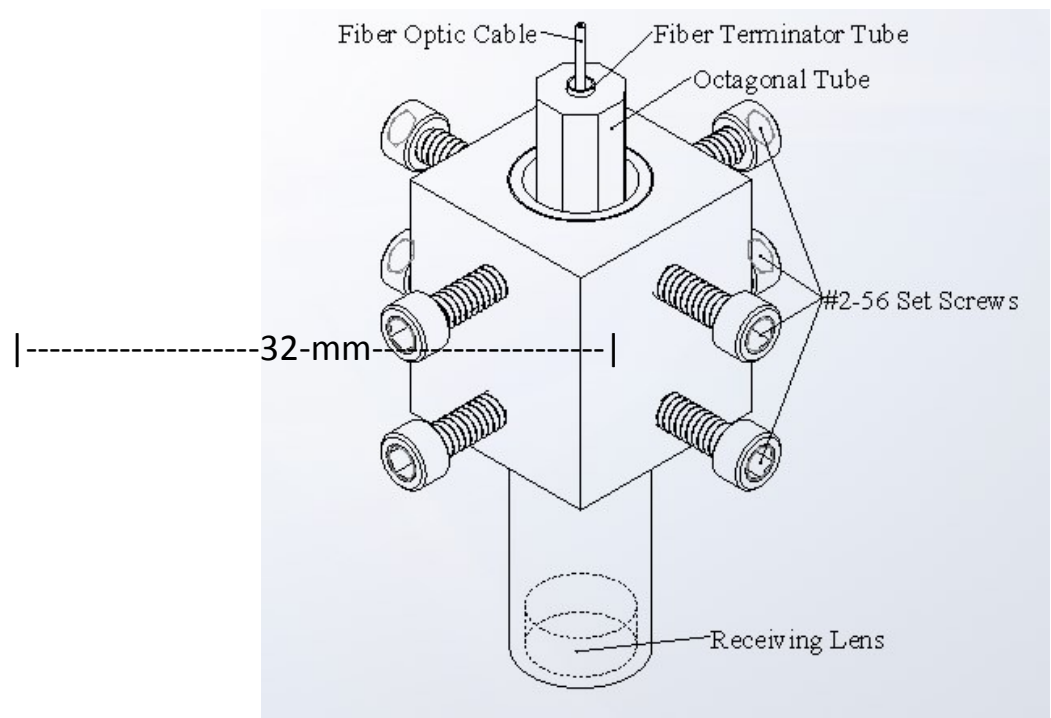

Figure 5.5 Receiving optics assembly.

The final piece of the probe is the probe head. This part consists of a 0.276 -in $(7-\mathrm{mm})$ diameter tube, 6.00-in (152.4-mm) in length, with the focusing lens from Table 5.1 glued into the end of it. This is the most important part of the probe as it was the component kept as small as possible which was the main purpose of this probe. This tube was glued into the main probe structure to complete the construction of the probe. The final probe design is shown in Figure 5.6.
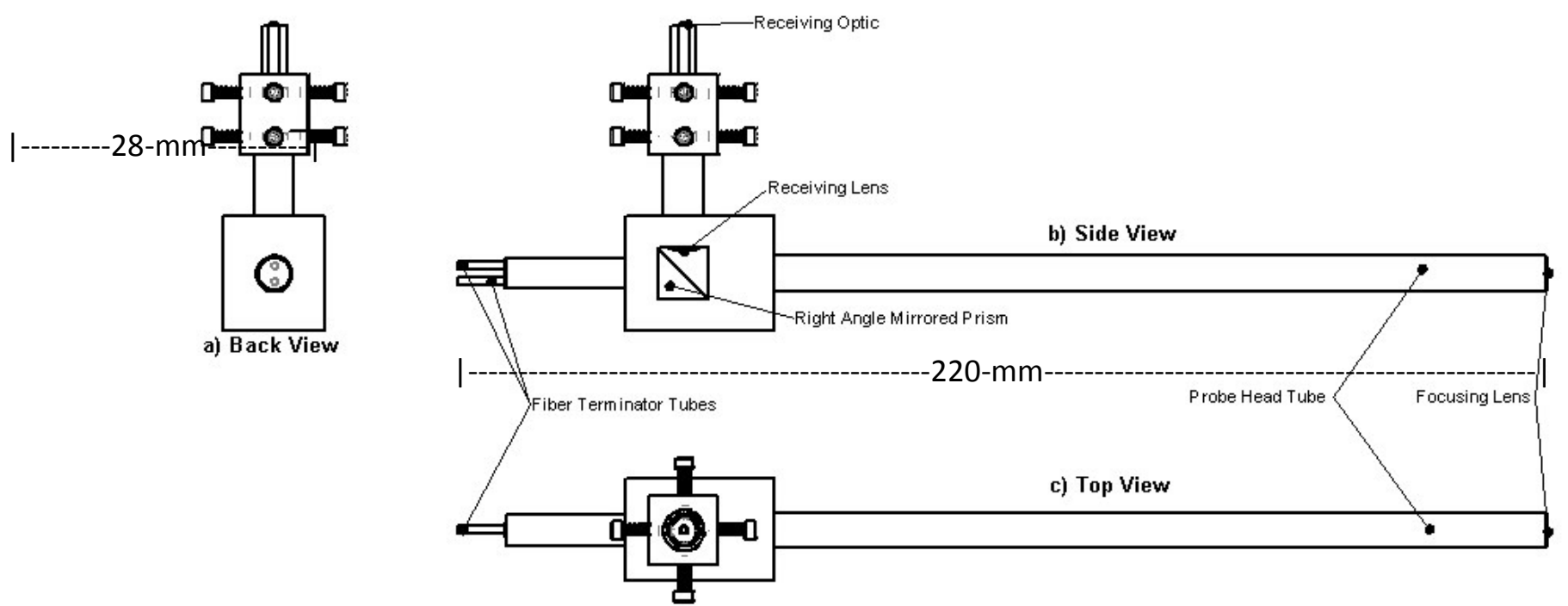

Figure 5.6 Borescopic LDV probe. a) probe as viewed from the back.

b) probe as viewed from the side. c) probe as viewed from the top. 


\subsection{ALIGNMENT}

Aligning the probe proved to be the hardest and most time consuming aspect of the construction process. This was due to the small parts needing to be extremely precise in their placement and the nature of glue contracting while drying. The process, however, was fairly straightforward. First, the two collimated transmitting beams had to be focused by pulling the terminator tubes in and out until the beams came to a point at a far distant wall. Next, a microscope objective was placed at the focal length of the focusing lens. Now the two beams passing through the microscope objective had to be made to overlap onto each other at the focal point of the transmitting lens. This was done by twisting the terminator tubes and adjusting their holders as needed. The beams emerging from the microscope objective were observed at a distant wall, which made it easy to observe the crossing of the beams. Once the beams overlapped, the tubes were glued into place. The next step was to send a beam through the receiving optics and make it overlap the other two beams as well. This was much easier as the set screws gave very precise control over the fiber. Once all three beams were crossed, the probe was ready for use. 


\section{EXPERIMENTAL SETUP}

The LDV system used for this project included the on-table optics, the borescopic LDV probe, and data acquisition and reduction units. A diagram for the system is shown in Figure 6.1. The on-table optics were used to create the two laser beams required for velocity measurements and to couple them with fiber-optic cables that transferred the beams to the borescopic probe. The probe was used to generate the measurement probe volume and to collect the light scattered by the seeding particles as they passed through the probe volume and couple the light into the receiving fiber-optic cable. This cable transferred the light to the data acquisition and reduction units.

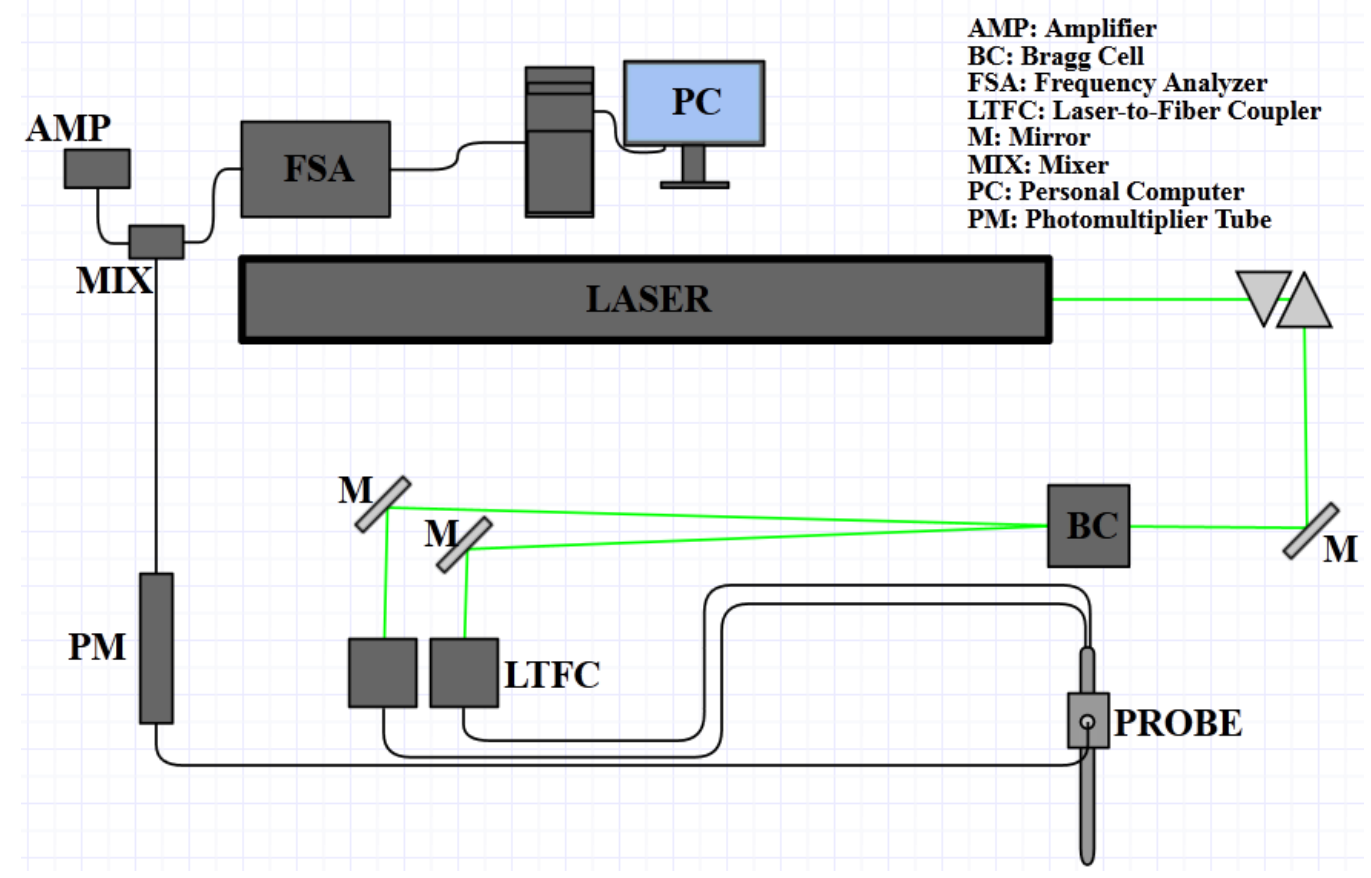

Figure 6.1 LDV System 
This chapter focuses on the components of the LDV system, the on-table optics, and the data acquisition and reduction units. First, the components of an LDV system are described in detail followed by a discussion regarding the on-table optics. Finally, the data acquisition and reduction units are discussed.

\subsection{COMPONENTS OF AN LDV SYSTEM}

An LDV system consists of a laser, transmitting and receiving optics, detectors, signal processors, and data reduction units. The laser was discussed in section 3.1. This section will focus on the transmitting and receiving optical components.

The transmitting and receiving optical components consist of the following:

- Mirrors

- Lenses

- Laser-to-Fiber Couplers

- Collimators

- Beam Splitters

- Polarizers

Transmitting optics of the system must be chosen carefully in order to generate the desired measurement volume and to achieve directional sensitivity to light. The transmitting optics first separate the laser beam emerging from the laser into the desired number of beams. They then apply the appropriate frequency shift to one of the beams to eliminate directional ambiguity. The optics then redirect the beams to fiber-optic cables, collimate the laser beams emerging from the fibers, and focus them to form the measurement probe volume. Each component will now be described in its own sub-section. 
$\underline{\text { MIRROR }}$

Mirrors are an important component of an LDV system. They are used for redirecting the laser beams in such a way that other components, such as lenses and beam splitters, can be easily located within the confines of the table used. Flatness, reflection loss, and damage threshold are the important properties to consider when choosing mirrors with the latter being the most important. The damage threshold of the mirrors must be higher than the power density of the laser beam.

\section{$\underline{\text { LENSES }}$}

Lenses are another important component of an LDV system. Lenses have multiple purposes in an LDV system. Some lenses are used to focus the laser beams to a desired focal point, while others are used to reduce a laser beam to a desired diameter. Lenses are also used to couple laser beams with fiber-optic cables and to collimate laser beams. The selection of lenses depends on the purpose they will serve, their size, and their focal length.

\section{LASER-TO-FIBER COUPLER}

Launching laser beams into fiber-optic cables with minimal losses is important and that is where laser-to-fiber couplers come in. LTFC are mechanical devices with up to six degrees of freedom adjustment capability. They house the fiber-optic cables and help to align the cables to receive the laser beams. 


\section{FIBER-OPTIC CABLES}

Fiber-optic cables are used to transfer laser beams from LTFC to the LDV probe and from the LDV probe to the photomultiplier tube. Single-mode and multi-mode fiber optic cables are commercially available and some are able to maintain the polarization of the light that forms the desired, undistorted fringe pattern in the measurement probe volume.

\section{COLLIMATORS}

Beam-waist (focus point of a Gaussian beam after a lens) adjustment and beam shaping are accomplished using collimators (Albrecht et al., 2003). Creating the desired measurement probe volume or achieving a specific beam-waist to launch a beam to a fiber-optic cable is accomplished using beam collimation. Highly disturbed measurement probe volumes result from uncollimated beams. Lenses and prisms are both commonly used to collimate laser beams. Figure 6.2 shows a two lens adjustable collimator. The adjustment comes from the ability to change the distance between the two lenses.

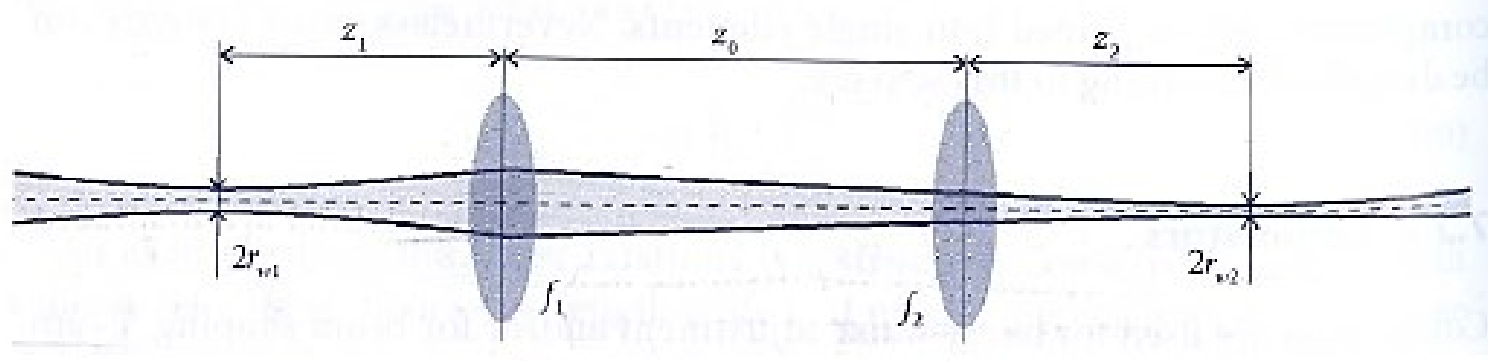

Figure 6.2 Dual lens adjustable collimator (Albrecht et al., 2003).

This type of collimator allows the expansion of the beam and the beam-waist to be adjusted. Equations 6.1 and 6.2 show how the distance, $\mathrm{z}_{2}$, and radius, $\mathrm{r}_{\mathrm{w} 2}$, are affected by the other variables in the above figure. 


$$
\begin{gathered}
z_{2}=\frac{f_{2}}{f_{1}}\left(f_{1}+f_{2}-z_{1} \frac{f_{2}}{f_{1}}\right) \\
r_{w 2}=r_{w 1}\left|\frac{f_{2}}{f_{1}}\right|
\end{gathered}
$$

where $f_{1}$ and $f_{2}$ are the focal lengths of the lenses.

The beam-waist in the measurement volume should be kept small $(50-100 \mu \mathrm{m})$ to achieve the desired system performance. The desired measurement probe volume is achieved by expanding the laser beams before they reach the front focal lens (Albrecht et al., 2003). Beam expansion and collimation are shown in Figure 6.3.

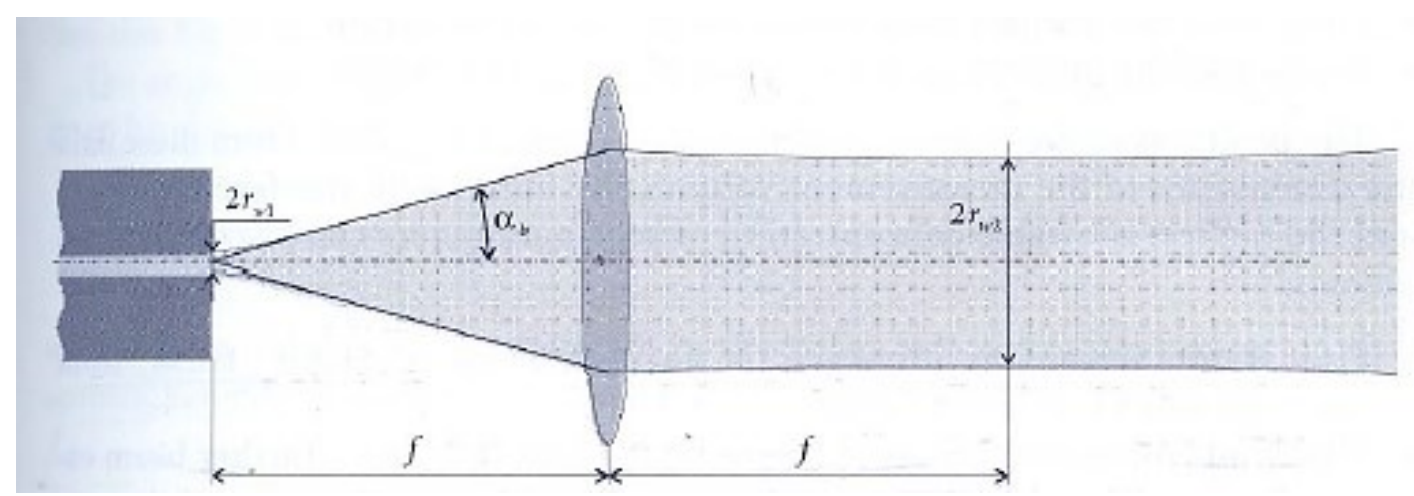

Figure 6.3 Beam expansion and collimation (Albrecht et al., 2003).

The divergence angle of the laser beam as it exits the fiber-optic cable is given by Equation 6.3.

$$
\alpha_{b} \approx \frac{\lambda}{\pi r_{c}}
$$

where $r_{c}$ is the core radius of the fiber-optic cable and $\lambda$ is the wavelength of the laser light. 
The desired beam expansion is achieved by choosing a lens with the correct focal length and positioning it at the correct location. The lenses used for collimation must be placed at their focal length away from the fiber-optic cables. The focal length, $f$, is chosen based on the desired collimated beam radius as seen in Equation 6.4.

$$
f=\frac{\pi r_{c} r_{w 2}}{\lambda}
$$

where $r_{c}$ is the core radius of the fiber-optic cable, $\lambda$ is the laser light wavelength, and $r_{w 2}$ is the desired radius of the collimated beam.

\section{BEAMSPLITTER}

Beamsplitters are used to split single laser beams into two or more beams. The split beams' intensity depends on the beamsplitter's split ratio. Most are designed to provide a 50/50 intensity split, splitting the beam into two beams of equal strength.

Bragg cells are the most common beamsplitters. Bragg cells divide a laser beam into two or more beams that are frequency shifted by the modulator frequency and its harmonics. The design usually uses the zeroth order, non-shifted, beam and the first order, first harmonic, beam.

The angle between the shifted beam and the non-shifted beam, $\alpha$, is given by Equation 6.5 .

$$
\alpha=\sin ^{-1} \frac{\lambda_{0}}{2 \Lambda_{a c}}
$$

where $\Lambda_{\mathrm{ac}}$ is the acoustic wavelength in the Bragg cell given by Equation 6.6.

$$
\Lambda_{a c}=\frac{V_{a}}{f_{s h}}
$$

where $V_{a}$ is the acoustic velocity in the Bragg cell medium, and $f_{s h}$ is the shift frequency. 


\section{$\underline{\text { POLARIZER }}$}

Only beams with the same direction polarization can create a true interference. This fact makes polarization of the laser light very important in an LDV system. Polarizers are used to reorient the polarization direction of laser beams. Polarizers consist of materials with anisotropic properties. Polarizers exhibit different refractive indexes in two orthogonal directions (Albrecht et al., 2003). One index is for the ordinary wave while the other is for the extraordinary wave. The assorted polarization states are shown in Figure 6.4.

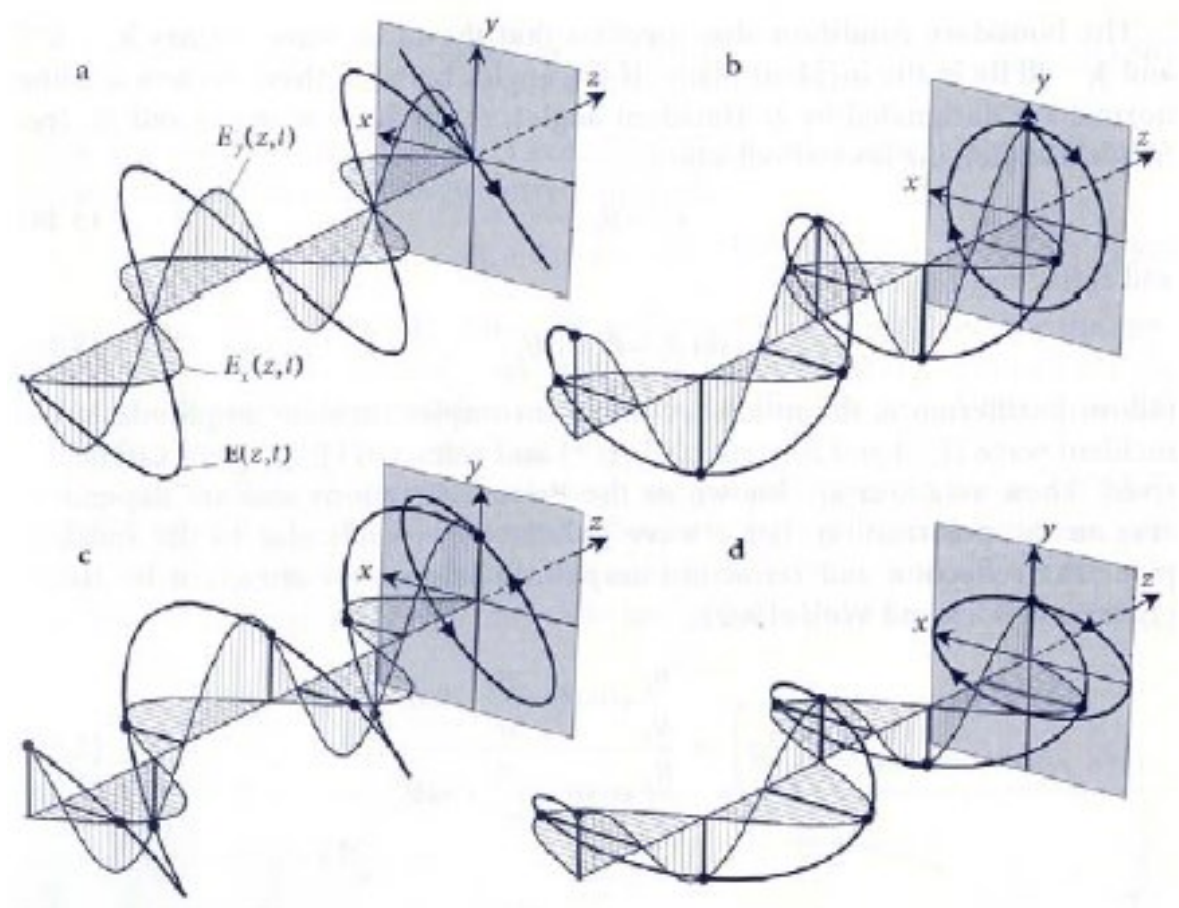

Figure 6.4 Polarization states (Albrecht et al., 2003).

a) $45^{\circ}$ linear. b) Right hand circular. c) left hand $45^{\circ}$ elliptical. d) Right hand $0^{\circ}$ elliptical. 
The phase difference determines the type of polarization. The phase difference, $\Delta \delta$, is given by Equation 6.7 .

$$
\Delta \delta=\left(n_{o}-n_{e}\right) d \frac{2 \pi}{\lambda}
$$

where $n_{o}$ is the ordinary wave, $n_{e}$ is the extraordinary wave, and $d$ is the thickness of the polarizer.

For example, one type of polarizer, a quarter-wave plate, has a phase difference of $\pi / 2$. Substituting into Equation 6.7 gives

$$
\left(n_{o}-n_{e}\right) d=\frac{\lambda}{4}
$$

The quarter-wave plate polarizer changes linearly polarized light to an elliptically polarized light.

Another type of polarizer, a half-wave plate, has a phase difference of $\pi$. Substituting this into Equation 6.7 gives

$$
\left(n_{o}-n_{e}\right) d=\frac{\lambda}{2}
$$

The polarization direction can be rotated in any direction using a half-wave plate.

Half-wave and quarter-wave plates are usually prior to the laser beams entering a beam splitter in a conventional LDV system. This allows the desired polarization to be achieved before the beam is split. This allows the transmitting optics and beam splitter to be rotated without having to rotate the laser. 


\subsection{ON-TABLE OPTICS}

As mentioned earlier, the on-table optics were used to create the two laser beams needed for velocity measurements. A schematic of the on-table optics used for the work done in this thesis is shown in Figure 6.5.

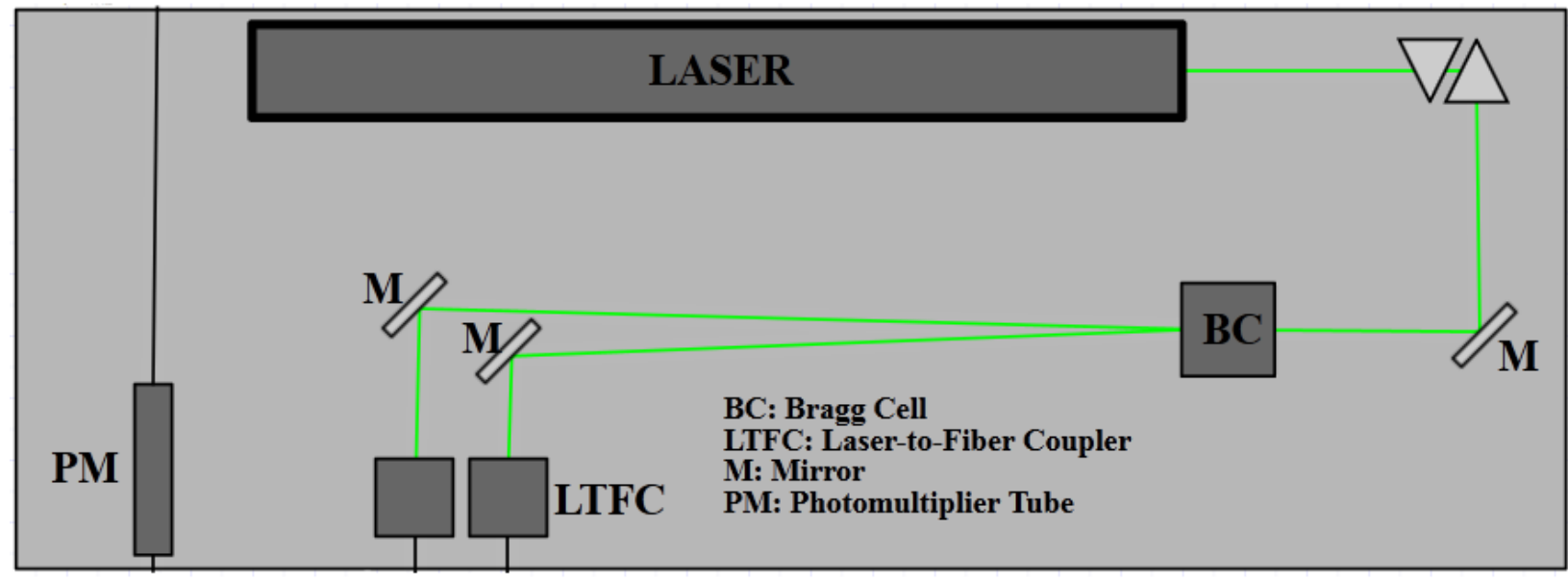

Figure 6.5 On-table optics.

The laser used as the light source for the work done in this thesis was a Spectra-Physics Ar-Ion laser (Innova 308C) with a maximum output of $8 \mathrm{~W}$-all-lines. The laser beam emerging from the laser has a diameter of 0.075 in $(1.9 \mathrm{~mm})$. It first passes through two prisms that turn the laser $90^{\circ}$ to keep the laser on the table. The laser beam then reflects $90^{\circ}$ off a mirror that directs it to a Bragg cell (IntraAction AFM-505A1). The Bragg cell splits the laser into two beams of equal strength. The zeroth order beam passes through without a frequency shift while the first order beam experiences a Bragg shift of 50MHz. Each beam then reflects off a separate mirror into a LTFC (Newport F-91-C1). The LTFCs are equipped with five-axis adjustment capabilities. A separate LTFC couples each laser beam separately with a fiber-optic cable (Corning PM 48-P-S) that then carry each beam to the LDV probe. The LDV probe is described in detail in Chapter 4 and will not be discussed again here. 


\subsection{DATA ACQUISITION AND REDUCTION UNITS}

The scattered light from the particles in the flow as they pass through the measurement probe volume is collected by the receiving optic within the probe where it is coupled with a multi-mode fiber-optic cable. The fiber-optic cable carries the collected light to a photomultiplier tube (Electron Tubes 9124SB) which converts the light information into an electrical signal. The frequency subtracted from the signal by the Bragg cell is then added to the electrical signal using a mixer and radio-frequency generator (B\&K 2005B RF). The signal is then fed to a frequency domain processor (TSI FSA-4000) which extracts the Doppler frequency information. A schematic showing this process is shown in Figure 6.6. A single channel was used on the frequency domain processor. The processor was attached to the data-acquisition and reduction computer via a firewire port.

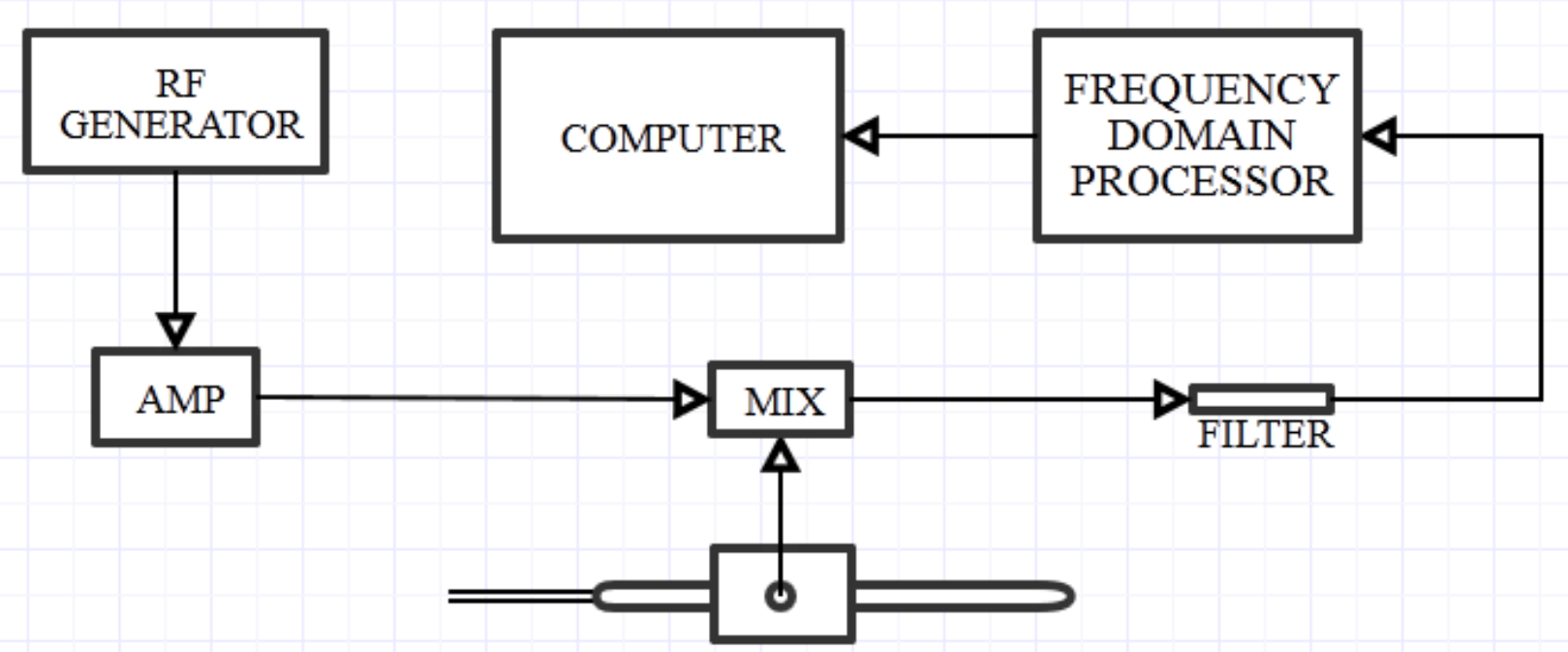

Figure 6.6 Data acquisition system 


\subsection{FLOW FIELD SETUP}

Two different flows were set up for this experiment. The first setup produced the pipe jet flow while the second setup produced the convergent nozzle jet flow. A particle seeding generator was used to provide the seeding for both flows.

\subsubsection{PARTICLE SEEDING GENERATOR}

As discussed in Chapter 4, accurate velocity measurements using an LDV system are highly dependent on the proper seeding of the flow. A TSI 9306 six-jet atomizer was used for providing the seeding to each of the flows. Di-Octyl-Phtalate was used as the seeding particle material. The atomizer generated particles with a mean diameter of $0.7 \mu \mathrm{m}$. The atomizer is shown in Figure 6.7.

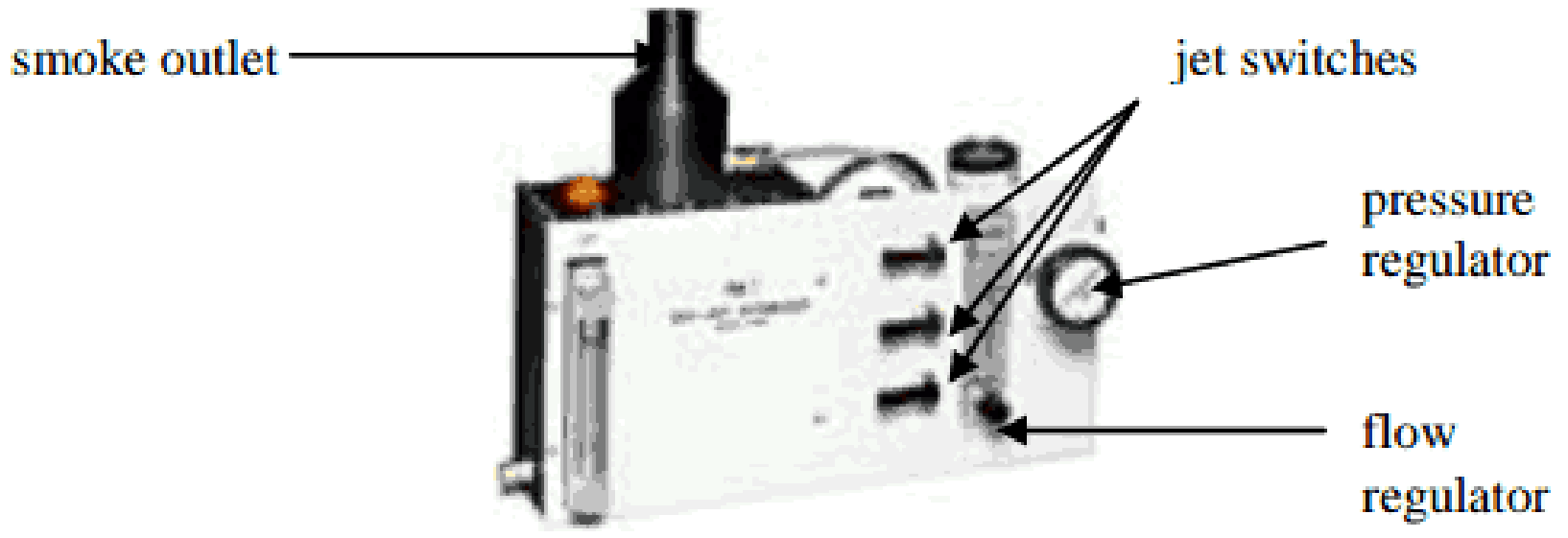

Figure 6.7 TSI 9306 six-jet atomizer

The generated smoke comes from the atomizer via a one inch diameter outlet on top of the device seen in Figure 6.7. The smoke then travels through a long rubber hose to the flow generator. The long rubber tube minimizes the amount of large oil particles that reach the flow because most of the large particles either collect on the walls of the tube or settle to the bottom. This makes the seeding particles delivered to the flow more uniform. This also slows the 
accumulation of oil particles on surfaces within the flow producers that could affect the flow field. The tube transfers the smoke to the settling chamber of the flow generators to assure adequate mixing of seeding particles with the flow before emerging from the jet exit.

Some effects of entrainment of air into the jet were not able to be measured with this setup due to the fact that only the emerging flow is seeded. This is known as in-homogenous seeding. The entire room encasing the flow would need to be seeded to acquire accurate measurements of the flow velocity at the edges of the jet. Fuchs et al. (1992) discuses the errors due to non-homogenous seeding. Their research shows that the bias error for the mean velocities at the edges of the jet can be shown to be as high as 3-5\% whereas the bias error is negligible just a few millimeters radially into the jet. Not much research exists on the errors at the jet edges for the turbulence quantities but they could be as high as $15 \%$.

\subsubsection{SETTING THE VELOCITY USING BERNOULLI'S EQUATION}

A manometer with one end attached to a pitot tube and the other exposed to the atmosphere is used to measure the total pressure of the flow. The total pressure read by the manometer is related to the velocity of the flow by Bernoulli's equation. The definition of the total pressure is the sum of the static and dynamic pressures which is represented by Equation 6.10.

$$
p_{0}=p_{s}+\frac{1}{2} \rho v^{2}
$$

where $p_{0}$ is the total pressure, $p_{s}$ is the static pressure, $\rho$ is the density of air, and $v$ is the velocity of air. The total pressure is read as gage pressure, the pressure difference between the total pressure and atmospheric pressure, and the static pressure is assumed to be atmospheric at 
the exit of the jets. Therefore, $p_{s}$ in Equation 6.13, is zero and $p_{0}$ is the pressure read from the manometer.

The manometer is read as inches of water and must be converted to a pressure. A change in height of the fluid within the manometer is related to a change in pressure as shown in Equation 6.14.

$$
\Delta p=\rho_{w} g_{c} \Delta h
$$

where $\Delta p$ is a change in pressure, $\rho_{w}$ is the density of water, $g_{c}$ is the acceleration due to gravity, and $\Delta h$ is the change in height of the manometer fluid. The manometer is first set to zero when there is no flow velocity by sliding the scale up or down. Once the flow has started, the velocity can be calculated from Equation 6.12 which is found using Equations 6.10 and 6.11 with $\Delta p=p_{0}$

$$
v=\sqrt{\frac{2 \rho_{w} g_{c} \Delta h}{\rho_{a}}}
$$

\subsubsection{PIPE JET FLOW SETUP}

The pipe chosen was a $4.54 \mathrm{ft}(1.38 \mathrm{~m})$ long pipe that was used to generate fully developed flow at the exit of the pipe. The length of the pipe was chosen to ensure that fully developed flow was achieved. The flow in the pipe was generated using a VACCON ejector pump. The flow entering the pipe was specifically not conditioned to obtain a relatively high turbulence intensity exit flow. The VACCON pump is a device that uses high-pressure compressed air as the motive air to entrain large sums of air through the use of the Coanda effect ( See Appendix D for more information of the Coanda effect). The flow speed at the exit of the 
pump is adjusted by changing the amount of motive air. The pump was used due to the ease of attaching it to the end of the pipe to achieve the desired flow field. The setup was originally envisioned to mimic the flow field at the exit of the test section of a model scale tunnel. This setup will be used to study the effects of the diffuser on the efficiency improvement of the Subsonic Aerodynamic Research Laboratory Tunnel of the Air Force Research Laboratories on 1:100 scale models. The setup was designed to mimic an exit flow equal to that of a uniform flow of $65 \mathrm{~m} / \mathrm{s}$ entering the pipe. A diagram of the pipe flow setup is shown in Figure 6.8 .

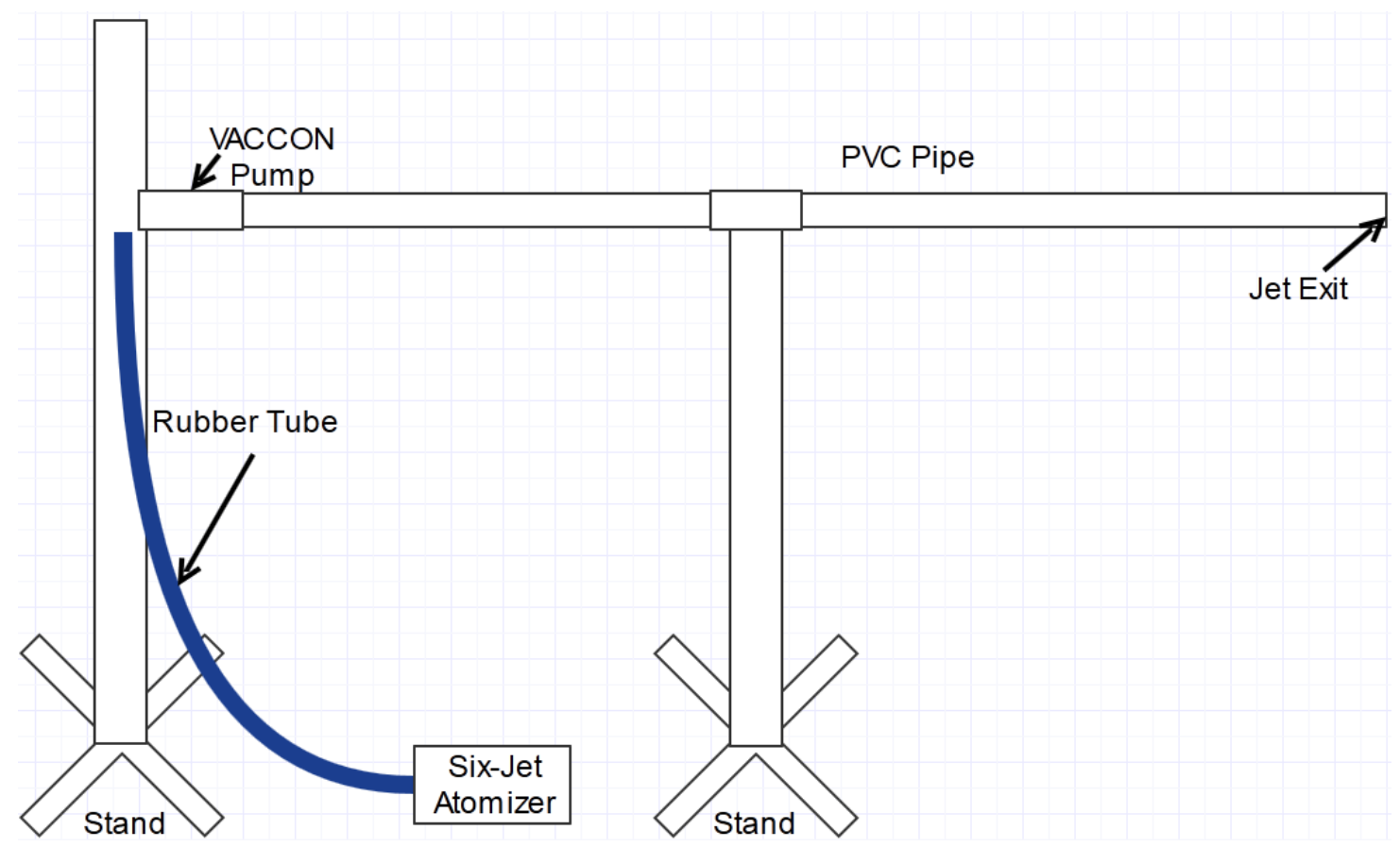

Figure 6.8 Diagram of pipe flow setup

It is well known that both the entrance length of the pipe and the fully developed exit flow profile depend on the Reynolds number of the flow based on the pipe diameter. The flow center velocity at the exit of the tunnel was planned to be set to $79.6-\mathrm{m} / \mathrm{s}$ to generate an exit flow velocity profile corresponding to a $65-\mathrm{m} / \mathrm{s}$ uniform flow entering the pipe. However, due to limitations in the shop air supply, the flow field centerline velocity maxed out at $55-\mathrm{m} / \mathrm{s}$ close to the exit of the pipe. The smoke from the smoke generator for seeding was fed to the VACCON 
pump by taping the rubber tube from the generator to a stand and setting the stand in such a way that the tube was as close to the inlet of the pump as possible without disturbing the inlet air flow. The velocity measurements were made in the pipe 0.118 -in $(3-\mathrm{mm})$ away from the exit of the pipe with the centerline velocity of the pipe set to 45,30 , and $15-\mathrm{m} / \mathrm{s}$.

The following equations give the relations between the entrance length and the exit velocity profile to the Reynolds number.

The entrance length for turbulent flow is defined as a function of Reynolds number (Engineering Toolbox, 2012).

$$
E I=4.4 R e^{1 / 6}
$$

and

$$
R e=\frac{V D}{v}
$$

where $D$ is duct inlet diameter, $V$ is the inlet velocity, and $v$ is the kinematic viscosity at the inlet. Substituting in the known values of the inlet velocity and kinmatic viscosity of air, a Reynolds $1.695 * 10^{5}$ was calculated for the desired $65 \mathrm{~m} / \mathrm{s}$ velocity. Using this value for the Reynolds number, the entrance length was calculated. To make use of this entrance length, another definition of the entrance length was used shown in Equation 6.15 (Engineering Toolbox, 2012).

$$
E I=\frac{l_{e}}{D}
$$

Substituting in the known values of the entrance length, $E I$, and inlet diameter, $D$, the total length of pipe to attain fully developed flow, $l_{e}$, was calculated to be $4.39 \mathrm{ft}(1.34 \mathrm{~m})$. 


\subsubsection{CONVERGENT NOZZLE JET FLOW}

A TSI Model 1125 hot-wire anemometry calibrator was used to produce the convergent nozzle jet. Air is provided to the calibrator by a pressurized air line from an air compressor. A diagram of the calibrator is shown in Figure 6.9.

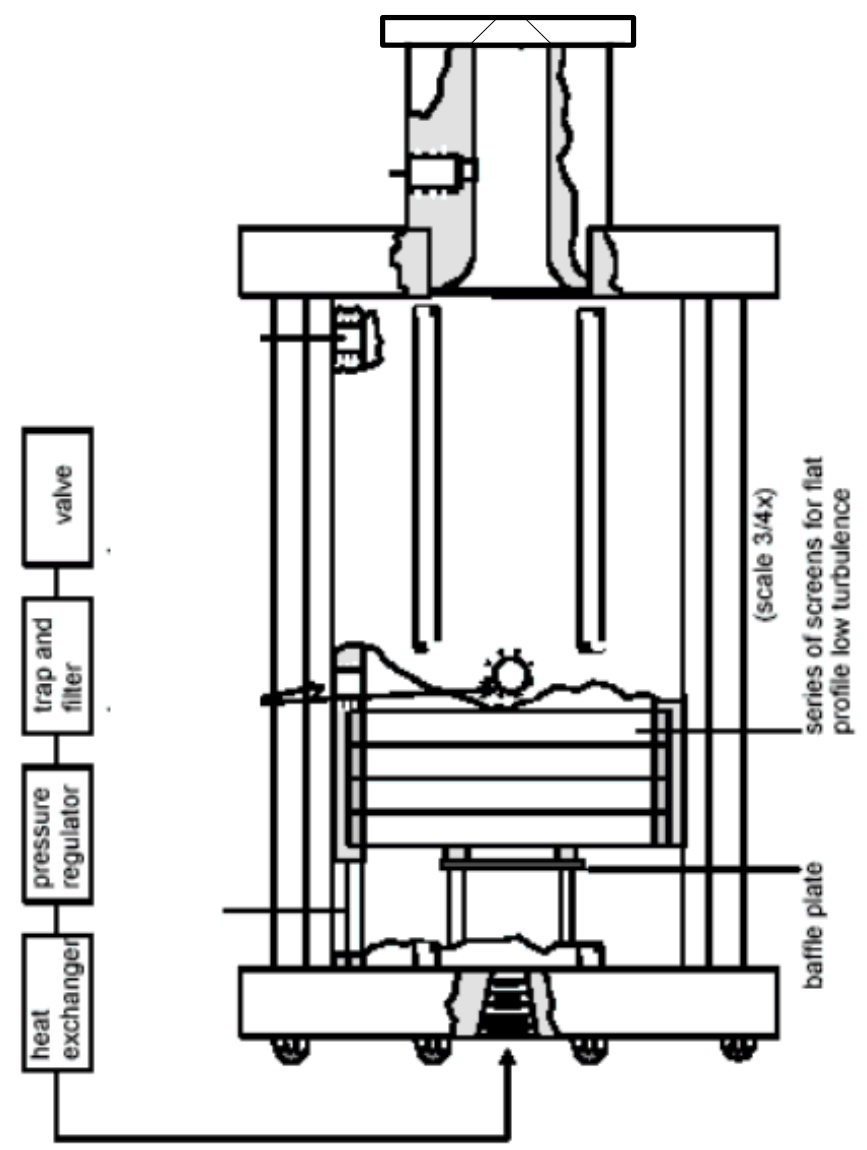

Figure 6.9 TSI Model 1125 hot-wire anemometry calibrator

First, air is brought to a shut off valve from an air compressor where it then travels through a trap and filter. The air then passes through a pressure regulator and flow regulator. The pressure regulator allows for large flow speed adjustments while the flow regulator allows smaller, more precise, flow speed adjustments. The flow then passes through a heat exchanger that is not used for the purposes of this research. The air leaving the heat exchanger passes into the settling chamber where it passes through five mesh screens to eliminate any swirling or other 
undesired motion. After passing through the screens, the flow is then delivered through a 0.60 -in $(15.24-\mathrm{mm})$ diameter pipe with a plate attached to it containing the 0.15 -in $(3.81-\mathrm{mm})$ diameter convergent nozzle. 


\section{EXPERIMENTAL PROCEDURE AND DATA}

Data reduction algorithms and the discussion of the data obtained in the current research are contained within this chapter. The LDV system and probe described in Chapters 5 and 6 were used to obtain all of the velocity data analyzed within this chapter. The probe developed was tested in two well-defined jet flows discussed in Chapter 6 to demonstrate that the probe could accurately measure a single velocity component of a flow. The probe was used to measure the $U$ velocity component of the jets. A total number of 30,000 data points were taken at each traverse location in both flows at varying data rates and efficiencies.

\subsection{DATA REDUCTION}

From the data taken at each measurement point, two variables were determined: $U$ - the mean axial velocity and $u^{2}$ - the mean normal stress. To determine the values of these variables, first, the standard deviation of the data was calculated; then all the outlier data points that were located greater than three standard deviations away from the mean were removed. Next, the mean velocities and fluctuating velocities were calculated using a residence time weighting scheme. Equation 7.1 shows the calculation of the mean axial velocity using the method described above. 


$$
\bar{U}=\frac{\sum_{i=1}^{N} U_{i} \Delta t_{i}}{\sum_{i=1}^{N} \Delta t_{i}}
$$

where $\bar{U}$ is the mean axial velocity, $U_{i}$ is the recorded axial velocities, $\Delta t_{i}$ is the length of time a particle spends in the measurement volume (known as the gate time), and $\mathrm{N}$ is the number of data points.

Once the mean velocities were calculated, the fluctuating velocities, known as the axial normal stress, are then calculated using Equation 7.2.

$$
u^{2}=\frac{\sum_{i=1}^{N}\left(U_{i}-\bar{U}\right)^{2} \Delta t_{i}}{\sum_{i=1}^{N} \Delta t_{i}}
$$

\subsection{PIPE JET FLOW}

The probe was first tested in the pipe jet flow described previously. For this flow, the axial velocity profile was measured 0.118 -in (3-mm) away from the jet exit as previously stated. The probe was positioned at the top of the pipe, first, and then it was traversed downward until the full profile was taken. The velocity data obtained were the mean axial velocity, $U$, and the mean normal stress, $u^{2}$. The mean velocities are non-dimensionalized by the mean axial velocity at the center of the pipe and the distance from the center is non-dimensionalized by the diameter of the pipe.

The pipe center velocity at the exit was set to 45,30 , and $15-\mathrm{m} / \mathrm{s}$ corresponding to Renolds numbers of approximately $1.17 * 10^{5}, 7.82 * 10^{4}$, and $3.91 * 10^{4}$, respectively. The flow velocity was set using Bernoulli's equation described before. Figures 7.1, 7.2, and 7.3 show plots of the mean centerline velocity profile at each velocity setting. Also shown in each figure is a plot based on the one-seventh power law and a plot of a power law that more 
accurately predicts the experimental data taken. The more accurate plots were obtained by calculating the left hand side of Equation 3.2 based on the experimental data and solving for $n$. The calculated values of $n$ at each velocity were, 7.532, 7.837, and 6.642 respectively. It is seen in the plots that the axial velocity is highest at the center of the pipe and decreases slowly as the distance from the center increases until the velocity rapidly decreases at the edges of the pipe.

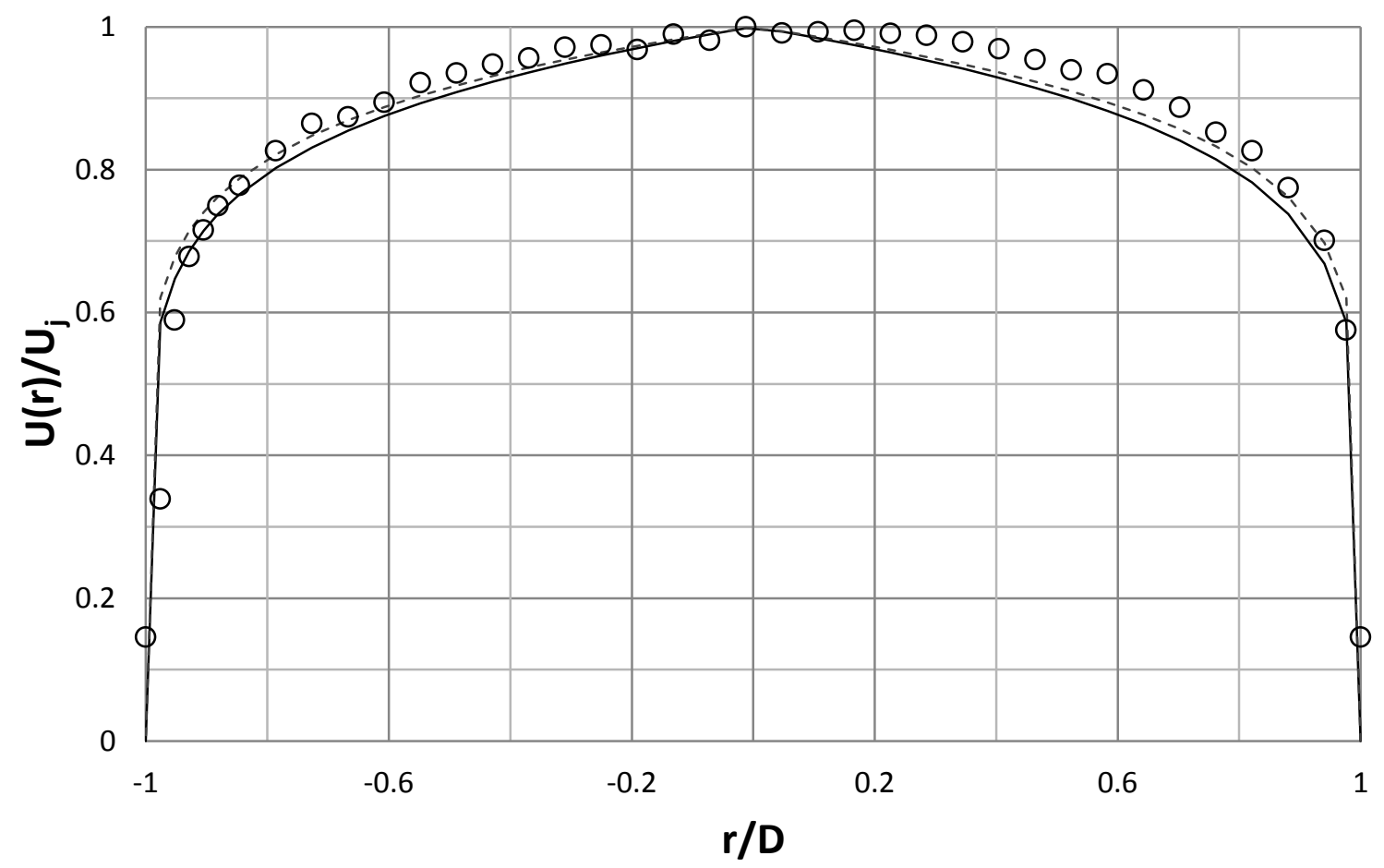

Figure $7.145 \mathrm{~m} / \mathrm{s}$ centerline velocity profile.

○, Experimental Data; -, $\mathrm{n}=7$ Power Law; --- $\mathrm{n}=7.837$ Power Law. 


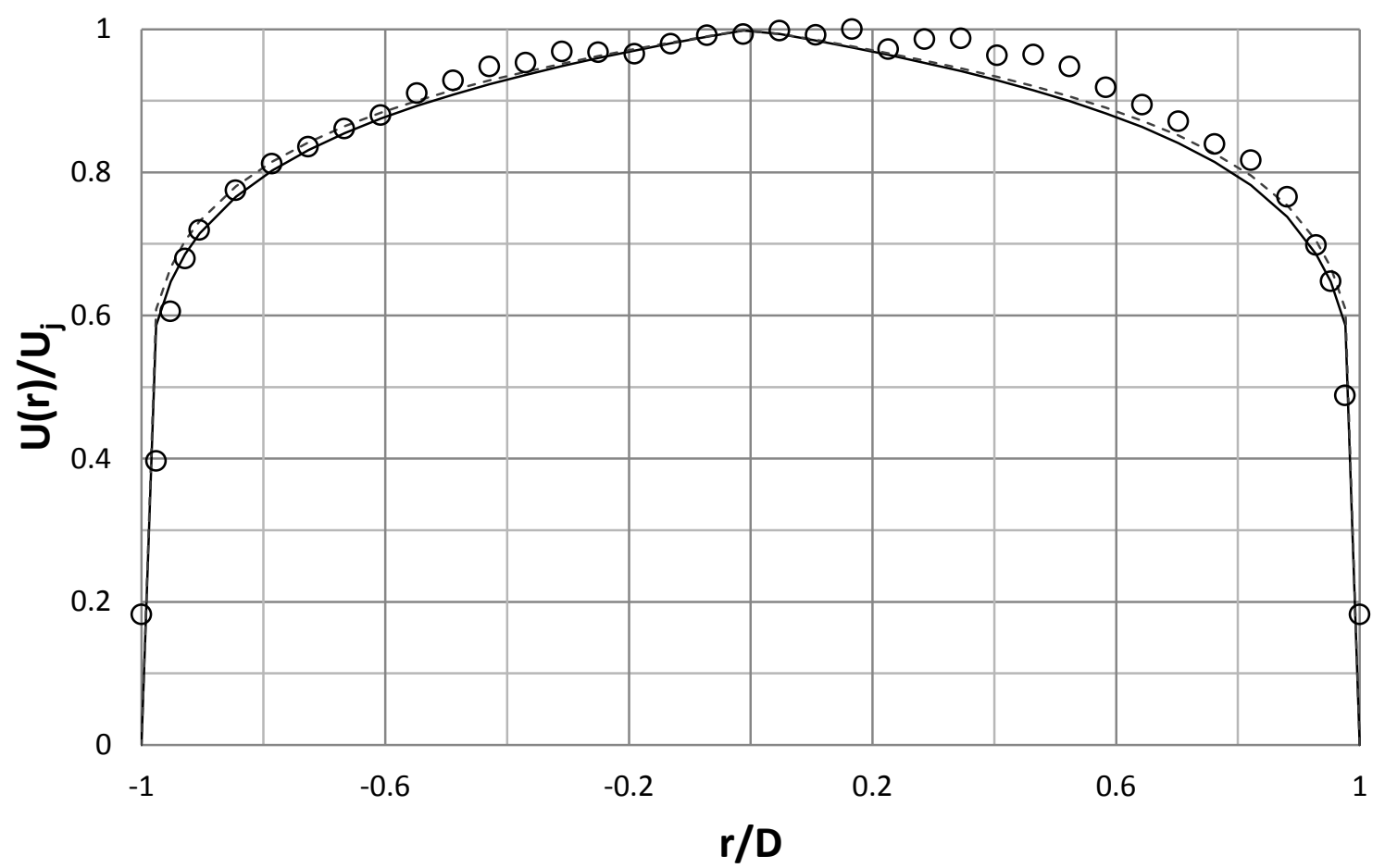

Figure $7.230 \mathrm{~m} / \mathrm{s}$ centerline velocity profile.

○, Experimental Data; -, $\mathrm{n}=7$ Power Law; ---, $\mathrm{n}=7.532$ Power Law.

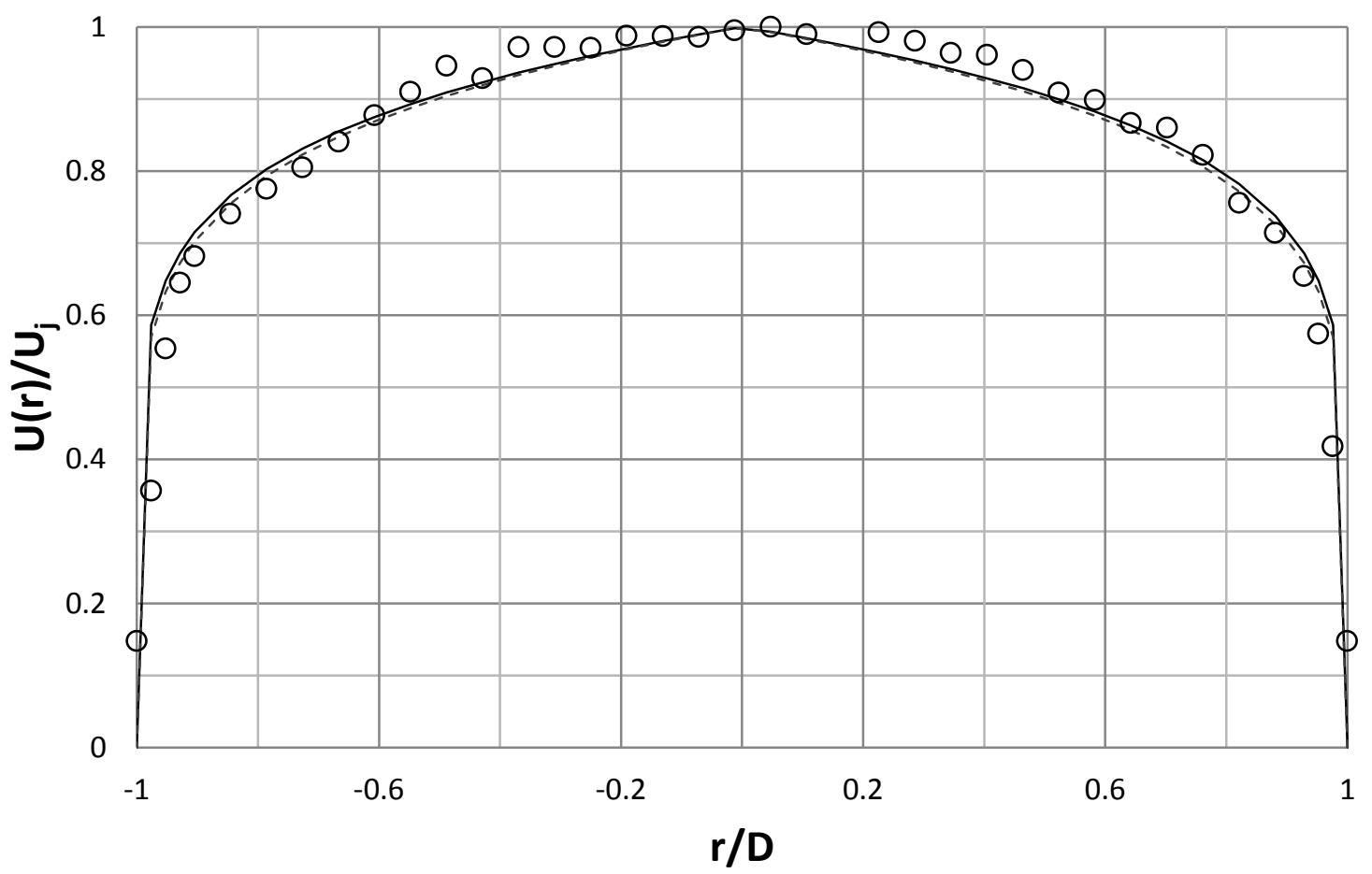

Figure $7.315 \mathrm{~m} / \mathrm{s}$ centerline velocity profile.

○, Experimental Data; -, $\mathrm{n}=7$ Power Law; ---, $\mathrm{n}=6.642$ Power Law. 
Since the jets are all axisymmetric, one side of the profile can be examined closer and it can be assumed that the other side matches it. This was done in Figures 7.4, 7.5, and 7.6 respectively to compare the data taken with the borescopic probe to power law solutions found in prior research. The data taken with the borescopic probe closely follows all the prior calculated power laws in the plot.

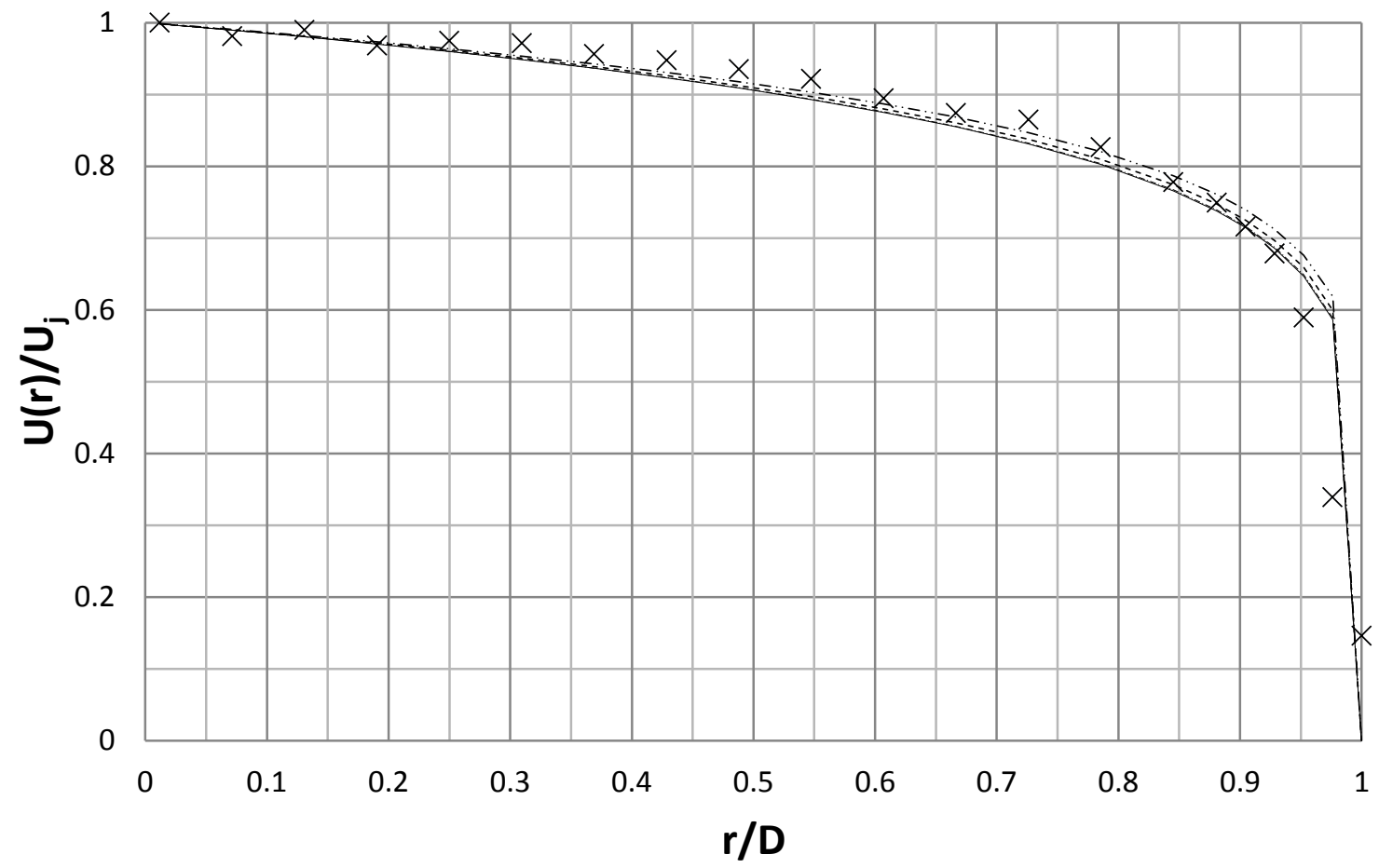

Figure $7.445 \mathrm{~m} / \mathrm{s}$ centerline velocity half profile.

$\times$, borescopic probe data; -, Blasius (1912); ---, Zagarola and Smitts (1998);

-•-, McKeon et al. (2004); -••-, Barrenblatt (1993). 


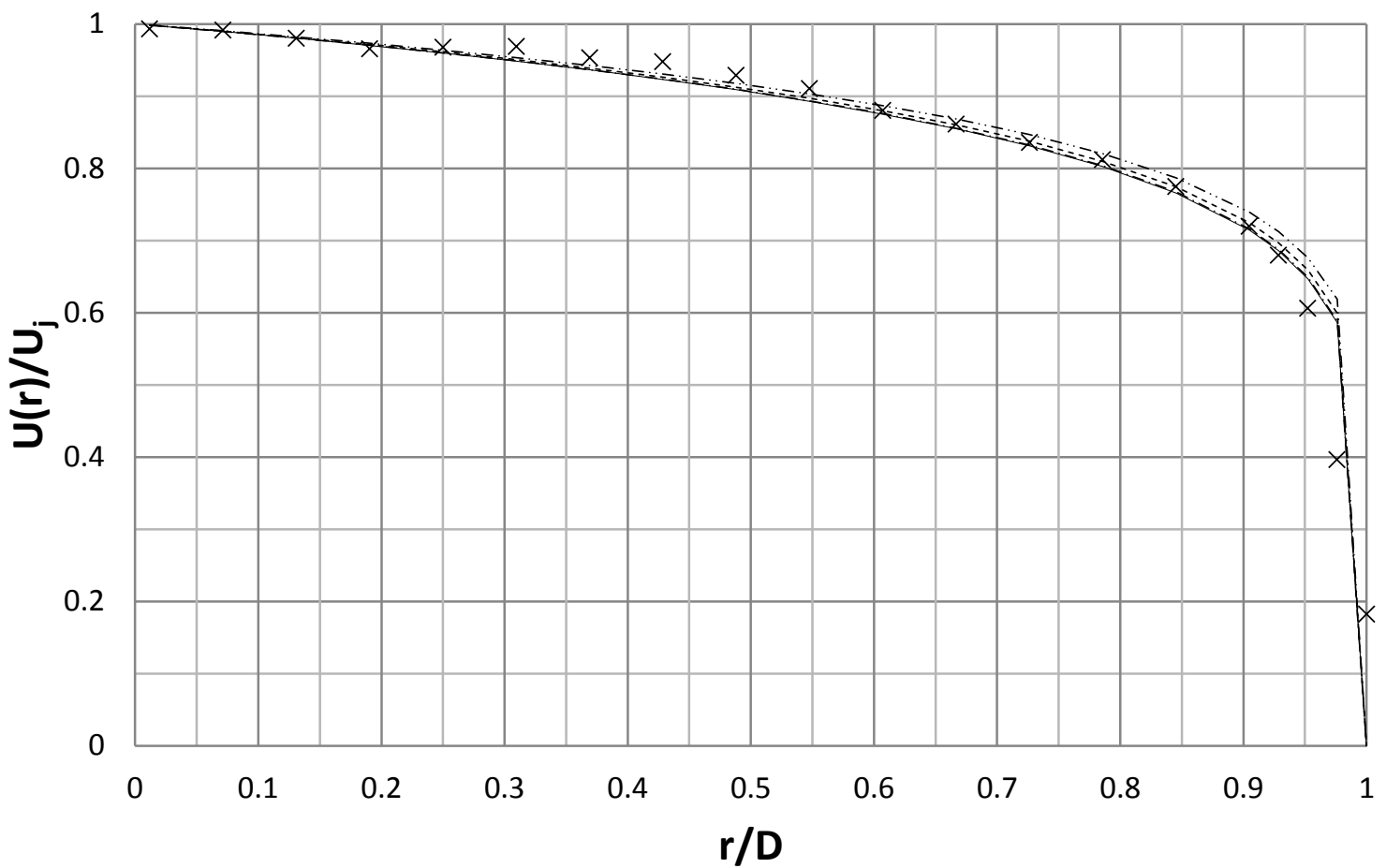

Figure $7.530 \mathrm{~m} / \mathrm{s}$ centerline velocity half profile.

$\times$, borescopic probe data; -, Blasius (1912); ---, Zagarola and Smitts (1998);

-•-, McKeon et al. (2004); -••-, Barrenblatt (1993).

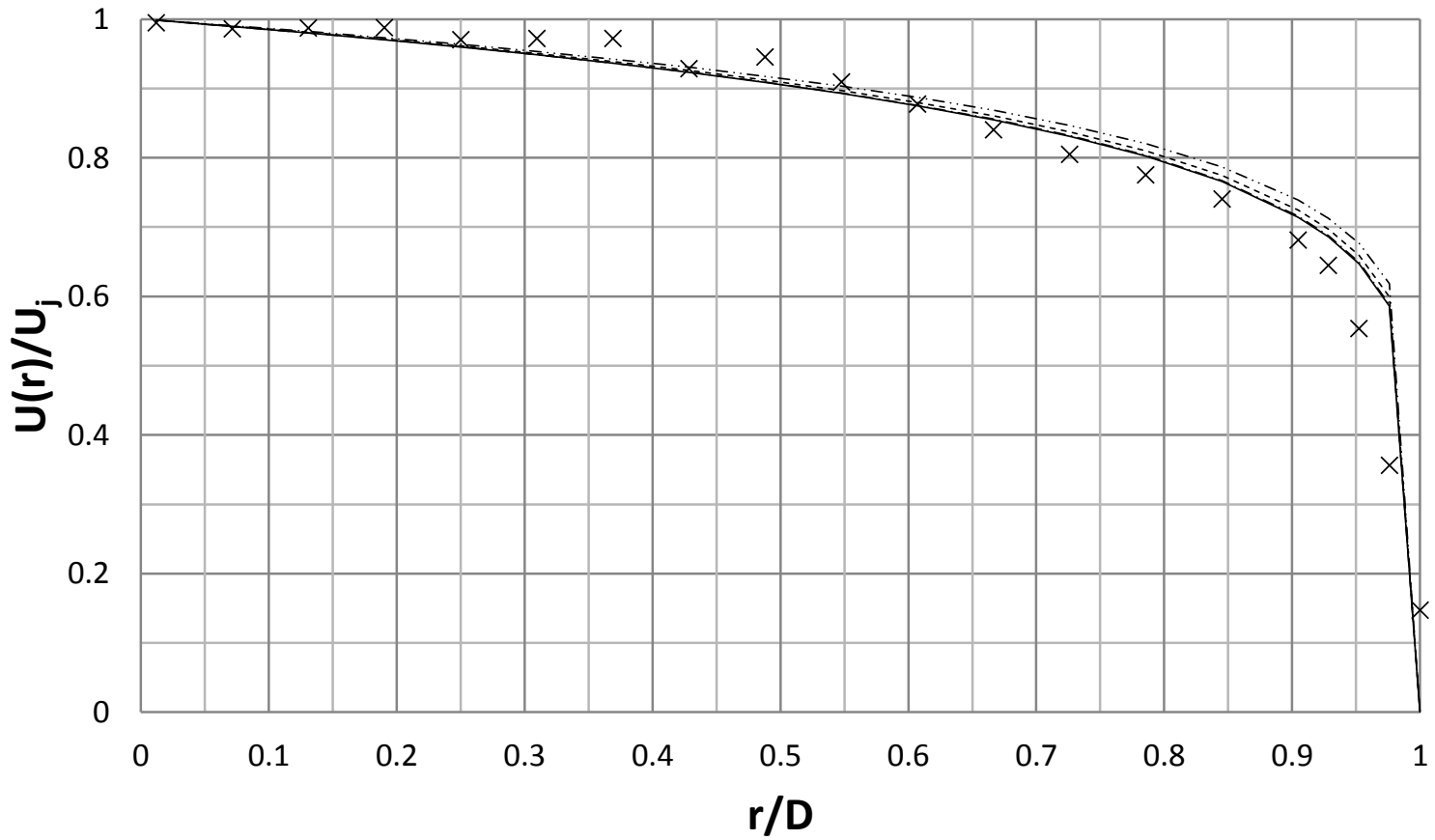

Figure $7.615 \mathrm{~m} / \mathrm{s}$ centerline velocity half profile.

$\times$, borescopic probe data; -, Blasius (1912); ---, Zagarola and Smitts (1998);

-•-, McKeon et al. (2004); -••-, Barrenblatt (1993). 
The root mean square of the fluctuating velocity in the axial direction, or the square root of the kinematic axial normal stress, is shown for each pipe center velocity in Figures 7.7, 7.8, and 7.9 respectively. As expected, the fluctuations are at a minimum near the center of the pipe and increase at the edges before rapidly decreasing to zero. The increase towards the edges is due to viscous effects and mixing of the jet with the surrounding ambient air. Away from the jet, at the edges, the fluctuation velocity values are zero since the jet is ejected to static air. However, the uncertainty in these values near the edge increases due to the inhomogenous seeding mentioned earlier. The velocity fluctuations presented were slightly higher than the fluctuations measured in previous research (Ferdman et al., 2000; Quin, 2005) due to the pipe flow used in this research. As mentioned earlier, the ejector pump was used without any flow conditioning to generate a pipe flow to mimic a high turbulence value jet. The higher velocity fluctuations were therefore expected.

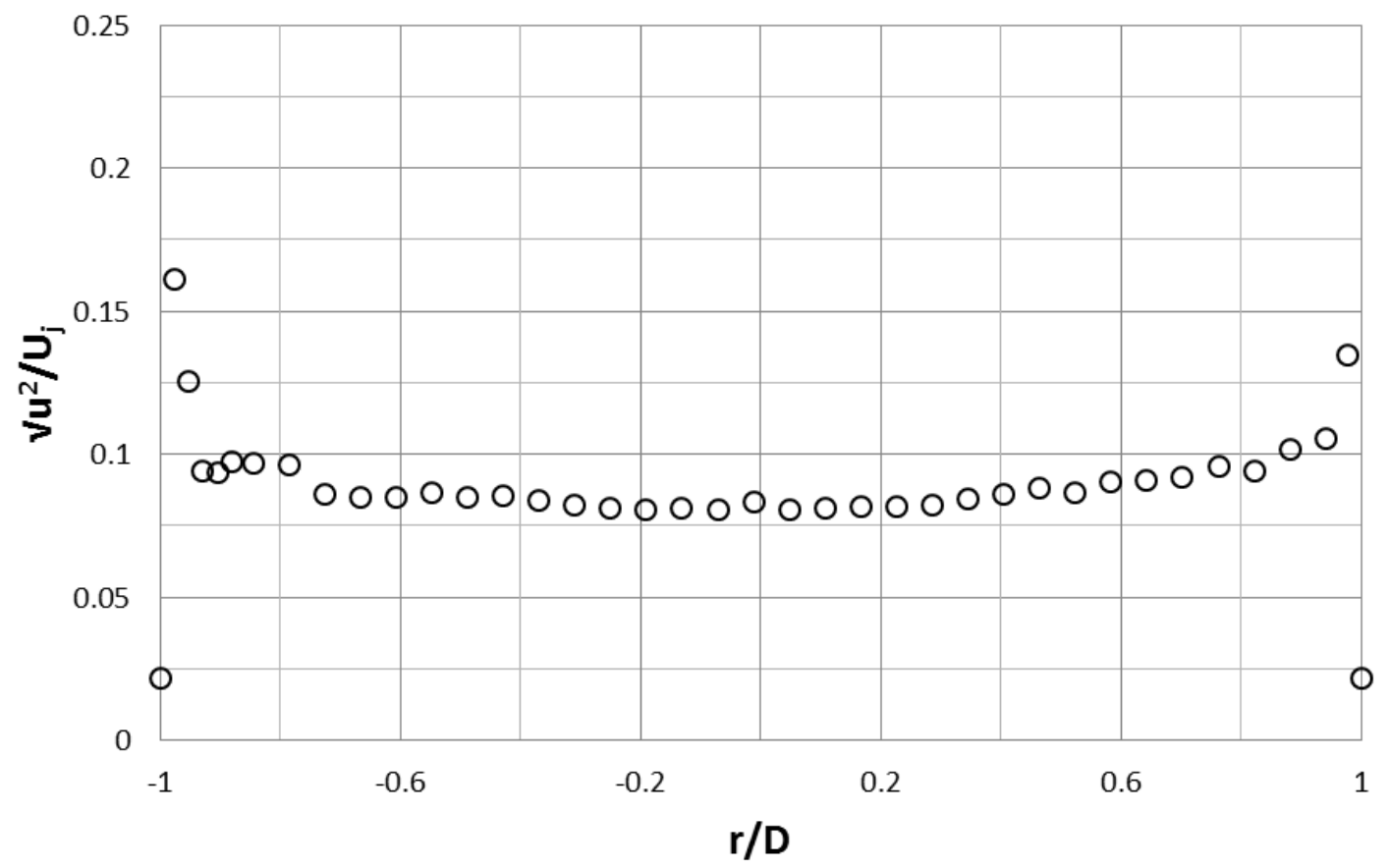

Figure $7.745 \mathrm{~m} / \mathrm{s}$ RMS of axial velocity fluctuations. 


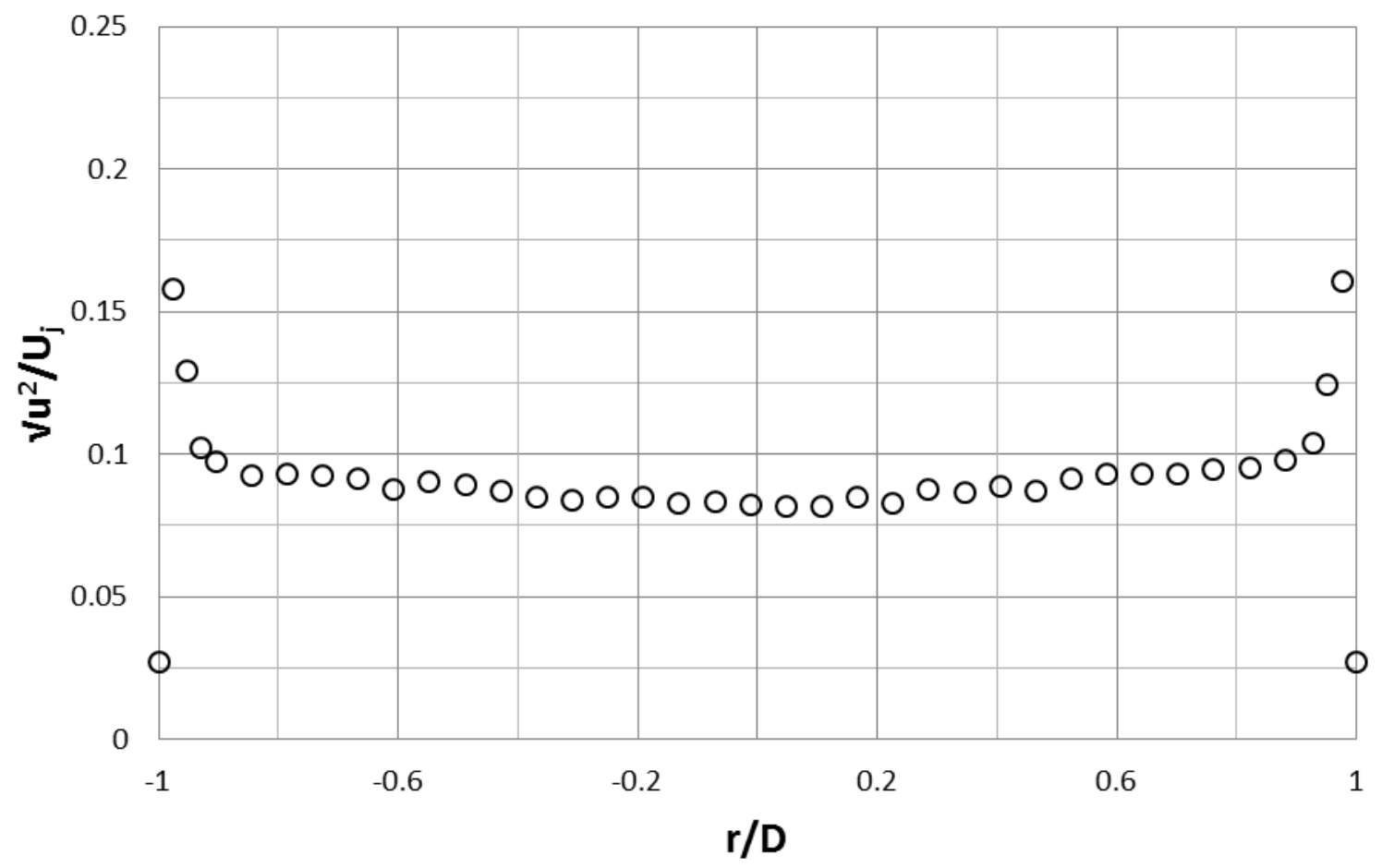

Figure $7.830 \mathrm{~m} / \mathrm{s}$ RMS of axial velocity fluctuations.

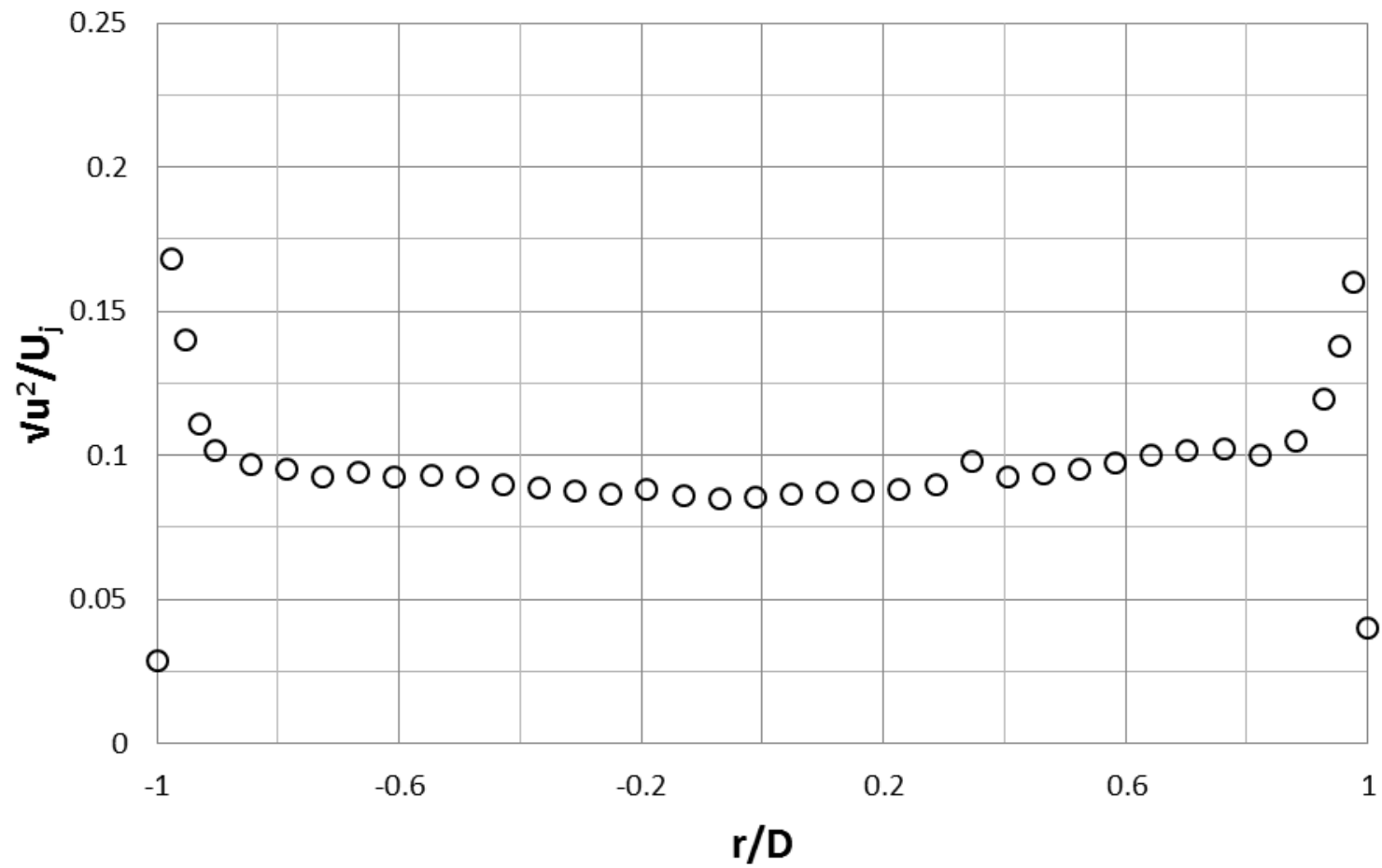

Figure $7.915 \mathrm{~m} / \mathrm{s}$ RMS of axial velocity fluctuations. 


\subsection{CONVERGENT NOZZLE JET FLOW}

The probe was next tested in the convergent nozzle jet flow described previously. For this flow, the center velocity decay was measured. This was accomplised by measuring the center velocity at various distances away from the jet exit plane. The flow was set up with the jet blowing upward with the probe set to traverse the flow along the center from the exit plane of the jet to 50 diameters above the flow.

The jet exit velocity was set to $75 \mathrm{~m} / \mathrm{s}$ corresponding to a Reynolds number of approximately $1.82 * 10^{4}$. Figure 7.10 shows the jet's center velocity decay with respect to distance away from the jet exit plane. The velocity was non-dimensionalized by the jet exit velocity and the distance from the jet exit plane by the diameter of the nozzle.

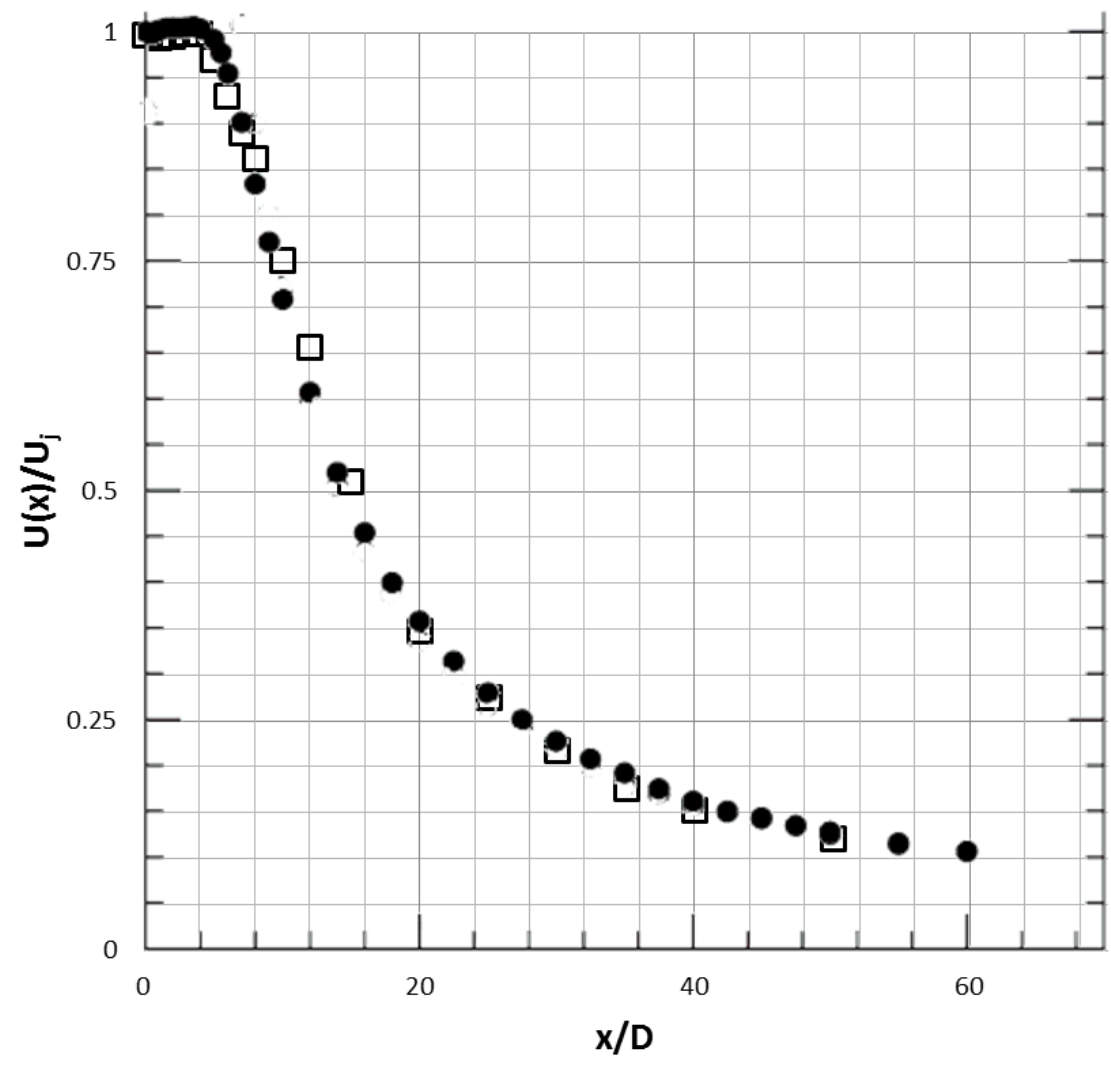

Figure 7.10 Velocity decay of $R e=2 * 10^{4}$ convergent nozzle flow. $\square$, borescopic probe data; $\bullet$, Quin (2005). 
It is seen from the figure that the data taken using the borescopic probe closely matches the data taken by Quin (2005). Quin (2005) conducted experiments using two separate jets, one generated by a contoured nozzle and another generated with a sharp edged nozzle, to study the difference between the flow fields near the jet exit at Reynolds number $1.84 * 10^{5}$.

Figure 7.11 shows the root mean square of the axial velocity fluctuations taken by the borescopic probe and by Quin. The data taken by the probe matches Quin's data within reason but slightly overshoots Quin's data. This overshoot is not unreasonable and most likely arises due to differences in the flow conditioning of the two.

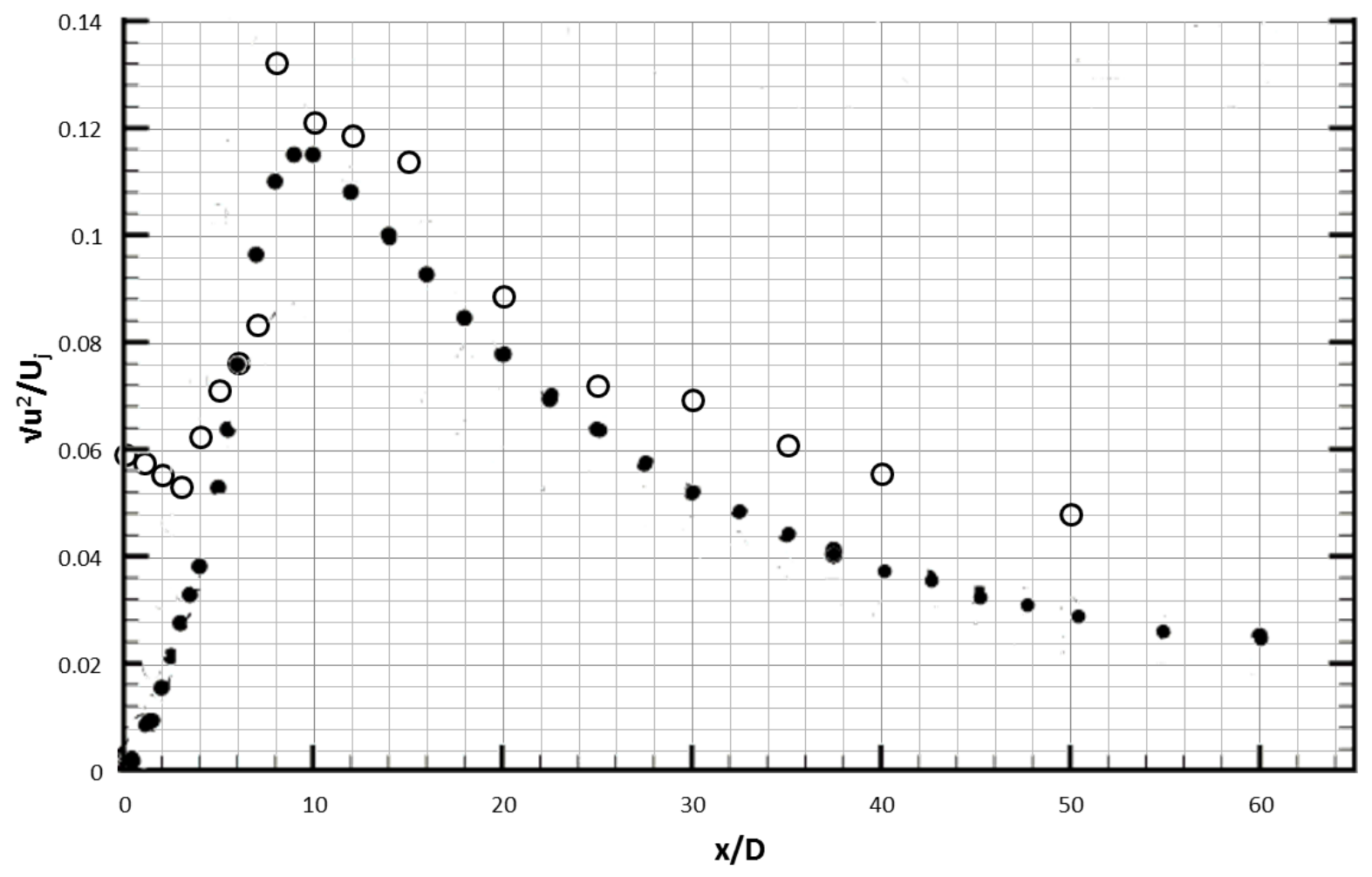

Figure 7.11 RMS of axial velocity fluctuations of $R e=10^{4}$ convergent nozzle flow. $\circ$, borescopic probe data; $\bullet$, Quin (2005). 
In an ideal round jet experiment, the velocity decay constant, $B$, and the spreading rate, $S$, do not vary with Reynolds number. Figure 7.12 compares the data taken to the line created when $B=5.8$ and $x_{0} / D=4.0$ which were the constants calculated using Hussein et al. (1994) experimental data as previously mentioned.

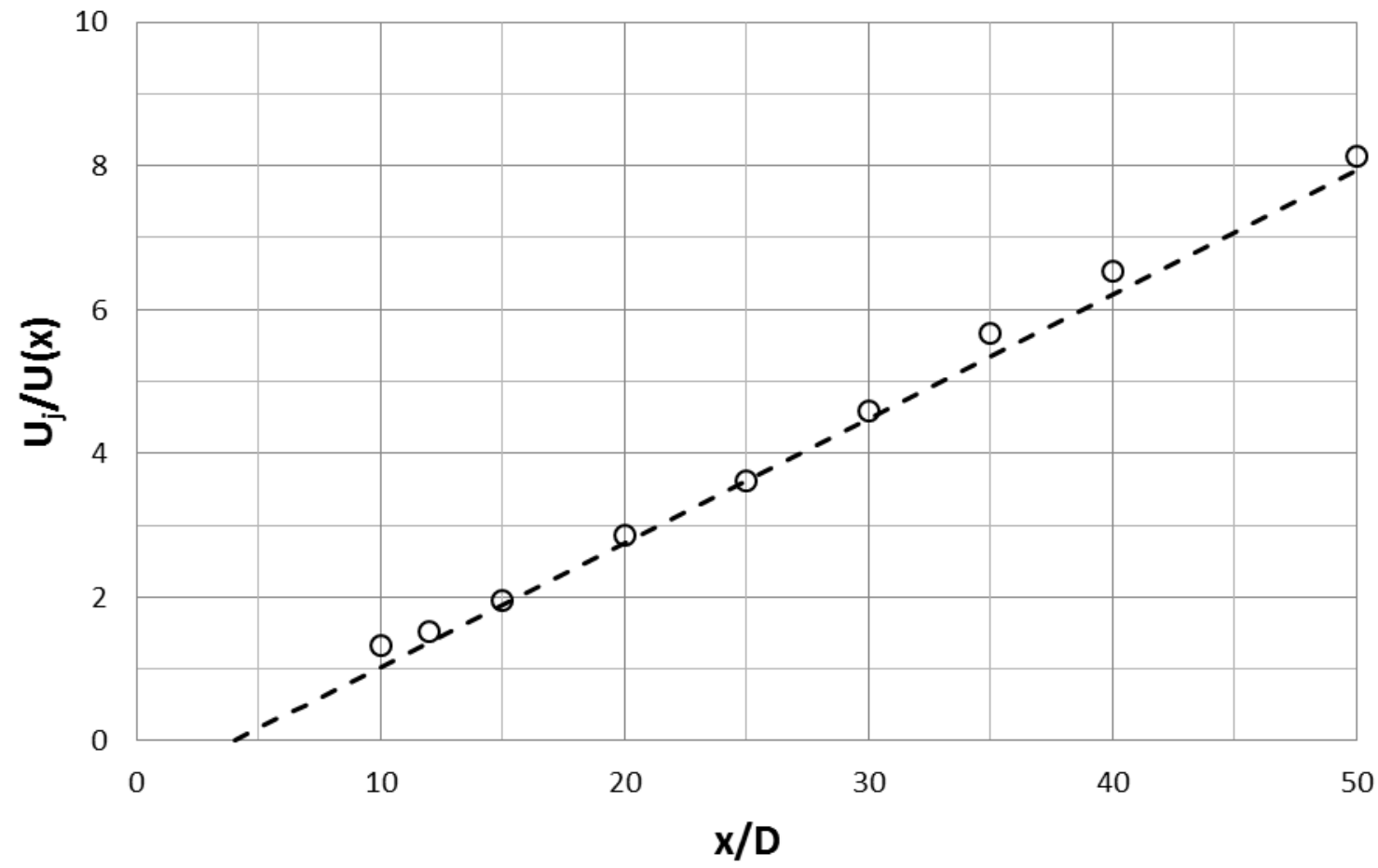

Figure 7.12 Variation of the mean axial velocity along along the centerline of the jet compared to the Equation 2.2 with $B=5.8$ and $x_{0} / D=4.0$ as found by Hussein et al. (1994). 


\subsection{UNCERTAINTIES}

Uncertainties in the collected data were determined using two separate data sets taken under the same conditions and using Chauvenet's criterion (Holman, 1978). Chauvenet's criterion is applied to elliminate outlying data. It specifies that a reading may be rejected if the probability of repeating the particular deviation from the mean is less than $1 / 2 n$ where $n$ is the number of readings. Equation 7.3 was used to calculate the standard deviation.

$$
\frac{d_{\max }}{\sigma}=1.15
$$

where $d_{\max }$ is half the difference between two measurements at the same location, $\sigma$ is the standard deviation, and 1.15 is the constant from Chauvenet's criterion. The uncertainties are presented for a $2 \sigma$ deviation calculated using Equation 7.3 in Table 7.1 which results in 21-1 odds of repeated measurements falling within this deviation.

Table 7.1 Uncertainty values for a $2 \sigma$ deviation

\begin{tabular}{|c|c|c|}
\hline & $U(x) / U_{j}$ & $\sqrt{u^{2}} / U_{j}$ \\
\hline Uncertainty & \pm 0.0339 & \pm 0.0271 \\
\hline
\end{tabular}




\section{CONCLUSION AND FUTURE WORK}

\subsection{CONCLUSION}

In this thesis, the development of a single-component, miniature, traversable, fiber-optic LDV probe has been described. The working principle of the probe is proven by comparison of data obtained using the probe in two different types of jet flows and data from previous studies. The Reynolds numbers of the flows varied from $1.82 * 10^{4}$ to $1.17 * 10^{5}$. In the jet flow experiments, the mean axial velocity profile of pipe jet flow and the centerline velocity decay of a convergent nozzle jet were measured. The kinematic normal stress was also measured for both flows.

For the pipe jet flow, the mean axial velocity was measured at thirty-nine different points along the centerline of the jet 3-mm away from the jet exit plane. The results obtained were determined to be in good agreement with previously published data.

For the convergent nozzle jet flow, the velocity at the center of the jet was measured at various distances from the jet exit plane to determine the jet decay rate. The results obtained were determined to be in good agreement with previously published data. 


\subsection{FUTURE WORK}

Suggestions for future research that emerged from this study are as follows:

- Observing how long the long-neck probe head tube can be while still obtaining reasonable results.

- Exchanging the 30-mm-focal-length transmitting lens with a $60-\mathrm{mm}$-focal-length lens

- Inserting the probe into a flow and determining if the probe has significant effects on the flow and comparing the results to another proven measurement technique such as hotwire anemometry.

For future construction, schematics of each part of the probe can be found in the appendix. 


\section{REFERENCES}

Afzal, N., Seena, A., Bushra, A. 2007. Power Law Velocity Profile in Fully Developed Turbulent Pipe and Channel Flows. ASCE. Journal of Hydraulic Engineering Vol. 133, 1080-1086.

Albrecht, H.E., Borys, M., Damashchke, N., Tropea, C. 2003. Laser Doppler and Phase Doppler Measurement Techniques. Springer.

Antonia, R.A., Zhao, Q. 2001. Effect of Initial Conditions on a Circular Jet. Experiments inf Fluids. Springer-Verlag. Vol. 31, 319-323.

Barenblatt, G.I. 1993. Scaling Laws for Fully Developed Turbulent Shear Flows I: Basic Hypothesis and Analysis. Journal of Fluid Mechanics, Vol. 248, 513-520.

Blasius, H. 1912. Ads Aechnlichkeitsgestz bei Reibungsvogangen. Z., Ver Drsch Ing., Vol. 56, No 16, 639-643.

Boersma, B.J., Brethouwer, G., Niuwstadt, F.T. 1997. A Numerical Investigation on the Effect of the Inflow Conditions on the Self-Similar Region of a Round Jet. American Institute of Physics. Physics of Fluids Vol. 10 No. 4, 899-909.

Byun, G., Olcmen, S.M., Simpson, R.L. 2004. A Miniature Laser-Dopler Velocimeter for Simultaneous Three-Velocity-Component Measurements. Measurement Science and Technology, Vol. 15, 2075-2082.

Engineering Toolbox. <www.engineeringtoolbox.com> 2012.

Esirgemez, E., Olcmen, S.M. 2005. A Spark-Plug LDV Probe for In-Cylinder Flow Analysis of Production IC Engines. Measurement Science and Technology, Vol. 16, 2038-2047.

Ferdman, E., Otugen, M., Kim, S. 2000. Effect of Initial Velocity Profile on the Development of Round Jets. Journal of Propulsion and Power, Vol. 16, No. 4, 676-686.

George, W.K. 1989. Self-Preservation of Turbulent Flows and Its Relation to Initial Conditions and Coherent Structures in Advances in Turbulence. Springer, New York.

Hryak, P., Lee, D.T., Gauntner, J.W., Livingwood, J.N.B. 1970. Experimental Flow Characteristics of a Single Turbulent Jet Impinging on a Flat Plate. NASA TN D-5690.

Hussein, H.J., Capp S., George W.K. 1994. Velocity Measurements in a High-Reynolds-Number, Momentum-Conserving, Axisymmetric, Turbulent Jet. Journal of Fluid Mechanics, Vol. $258,31-75$

Ivanchenko, O., Esirgemez, E., Olcmen, S.M. 2007. A Miniature Three-Component LDV Probe. Measurement Science and Technology, Vol. 18, 2014-2020.

Kassab, S.Z., Bakry, A.E., Warda, H.A. 1996. Laser Doppler Anemometry Measurements in an Axisymetric Turbulent Jet. Rev. Sci. Instrum., Vol. 67, No. 5, 1842-1849.

McKeon, B.J., Li, J., Jiang, W., Morrison, J.F., Smits, A.J. 2004. Further Observations on Mean Velocity Distribution in Fully Developed Pipe Flow. Journal of Fluid Mechanics, Vol. 501, 135-147. 
McKeon, B.J., Zagarola, M.V., Smits, A.J. 2005. A New Friction Factor Relationship for Fully Developed Pipe Flow. Cambridge University Press. J. Fluid Mech Vol. 538, 429-443.

Menon, R. 1982. Laser Doppler Velocimetry: Performance and Applications. America Laboratory.

Menon, R., Lai, W.T. 1991. Key Considerations in the Selection of Seed Particles for LDV Measurements. TSI Inc. Fourth International Conference of Laser Anemometry, Ohio.

Mi, J., Nathan, G.J., Nobes, D.S. 2001. Mixing Characteristics of Axisymmetric Free Jets from a Contoured Nozzle, an Orifice Plate, and a Pipe. ASME. Journal of Fluids Engineering. Vol. 123, 878-883.

Munson, B.R. 2002. Fundamentals of Fluid Mechanics, $4^{\text {th }}$ Edition. John Wiley \& Sons, 475-477

Microsoft Encarta Encyclopedia 2000.

Pai, S. 1954. Fluid Dynamics of Jets. D. Van Nostrand Company, Inc., New York.

Pope, S.B. 2000. Turbulent Flow. Cambridge University Press, USA.

Quin, W.R. 2005. Upstream Nozzle Shaping Effects on Near Field Flow in Round Turbulent Free Jets. European Journal of Mechanics B/Fluids Vol. 25, 279-301.

Toonder, J.M., Nieuwstadt, T.M. 1997. Reynolds Number Effects in a Turbulent Pipe Flow for Low to Moderate Re. American Institute of Physics. Physics of Fluids Vol. 9 No. 11, 3398-3409.

Watrasiewicz, B.M., Rudd, M.J. 1976. Laser Doppler Measurement. London.

Wygnanski, I., Fiedler, H.E. 1969. Some Measurements in the Self-Preserving Jet. Journal of Fluid Mechanics, Vol. 38, 577.

Vickers, J.M.F. 1959. Heat Transfer Coefficients Between Fluid Jets and Normal Surfaces. Industrial \& Engineering Chemistry Research Vol. 51, No. 8, 967-972.

Xu, G., Antonia, R.A. 2002. Effect of Different Initial Conditions on a Turbulent Round Free Jet. Springer-Verlag. Experiments in Fluids Vol. 33, 677-683.

Yeh, Y., Cummins H. 1964. Localized Fluid Measurements with a He-Ne Laser Spectrometer. Applied Physics Letters, Vol. 4, 176-178.

Zagarola, M.V., Perry, A.E., Smits, A.J. 1997. Log Laws or Power Laws: The Scaling in the Overlap Region. American Institute of Physics. Physics of Fluids Vol. 9 No.7, 20942100.

Zagarola, M.V. and Smits, A.J. 1998. Mean Flow Scaling in Turbulent Pipe Flow. Journal of Fluid Mechanics, Vol 373, 33-79. 
APPENDICES 


\section{APPENDIX A}

\section{A.1 MAIN PROBE STRUCTURE}
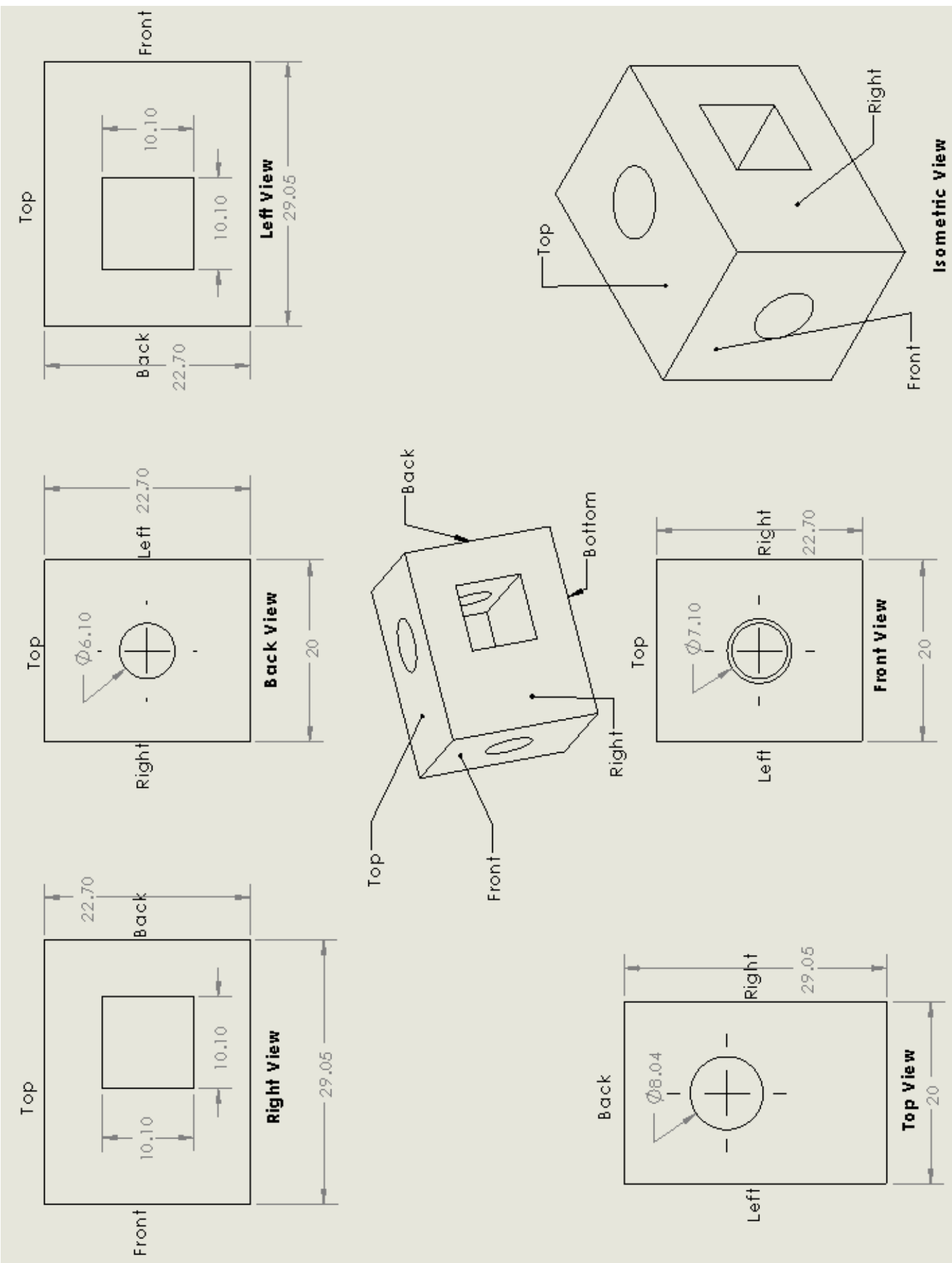

Figure A.1 Dimensioned mounting block [mm] 


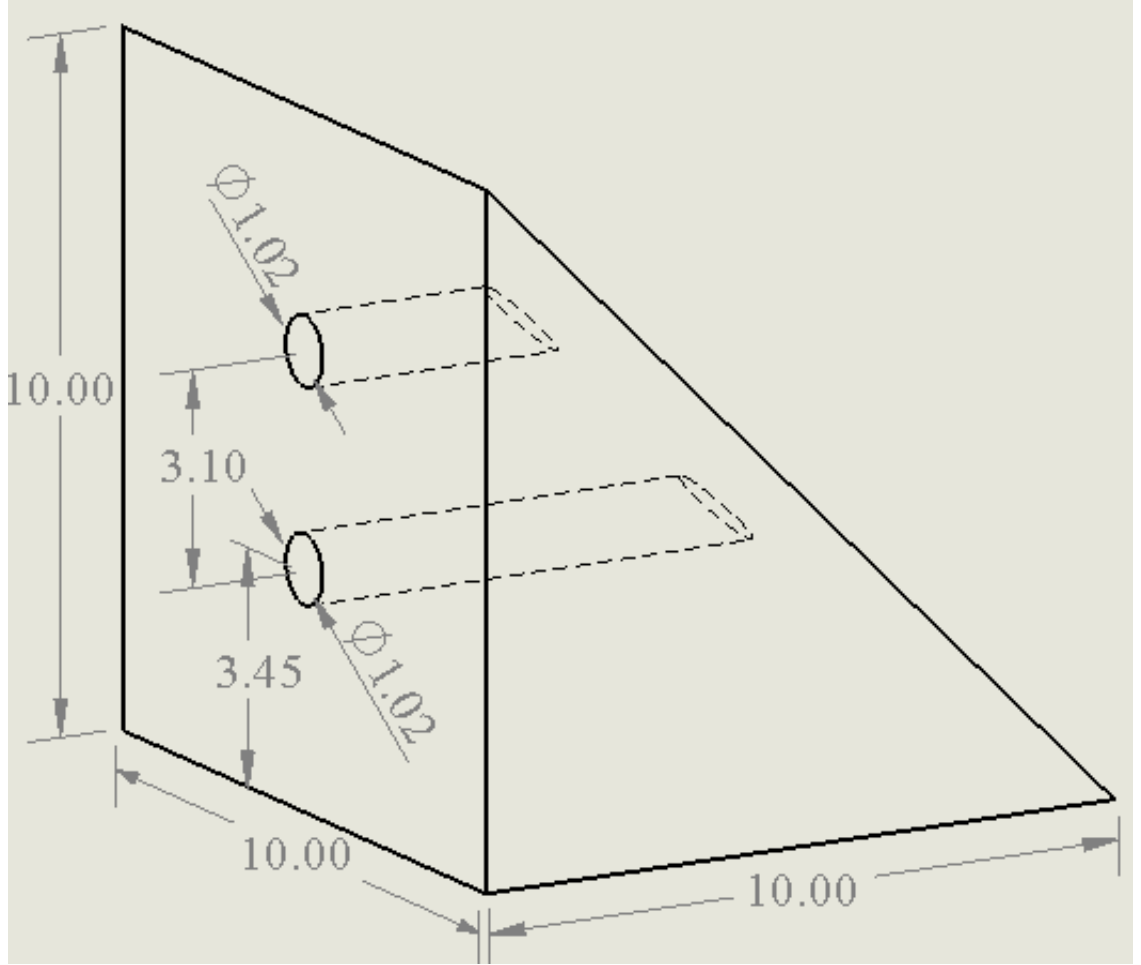

Figure A.2 Dimensioned mirrored wedge [mm]

\section{A.2 PROBE HEAD}

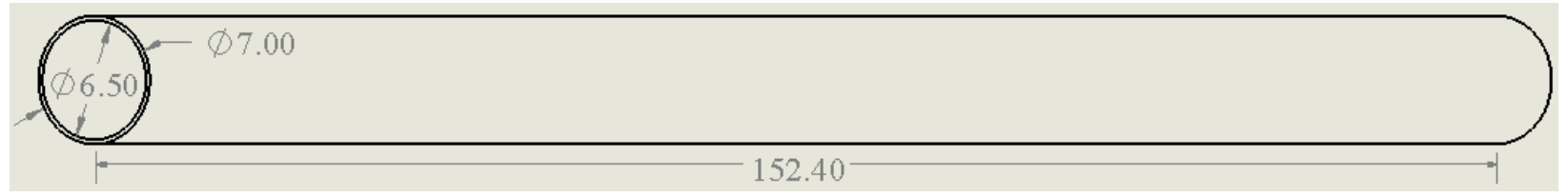

Figure A.3 Dimensioned long-neck probe head tube [mm]

\section{A.3 TRANSMITTING OPTICS}

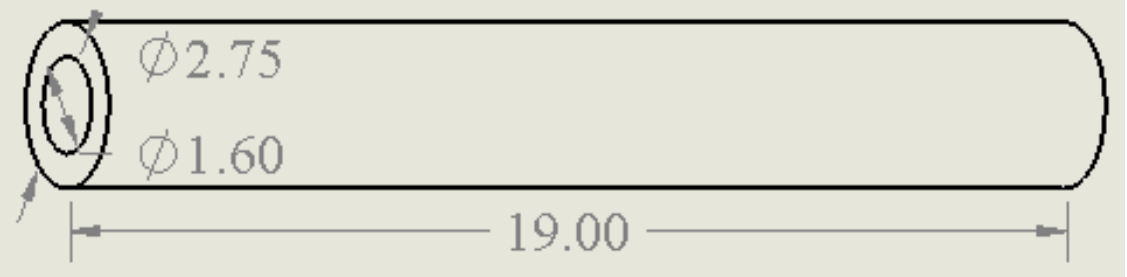

Figure A.4 Fiber terminator tube holder X2 [mm] 


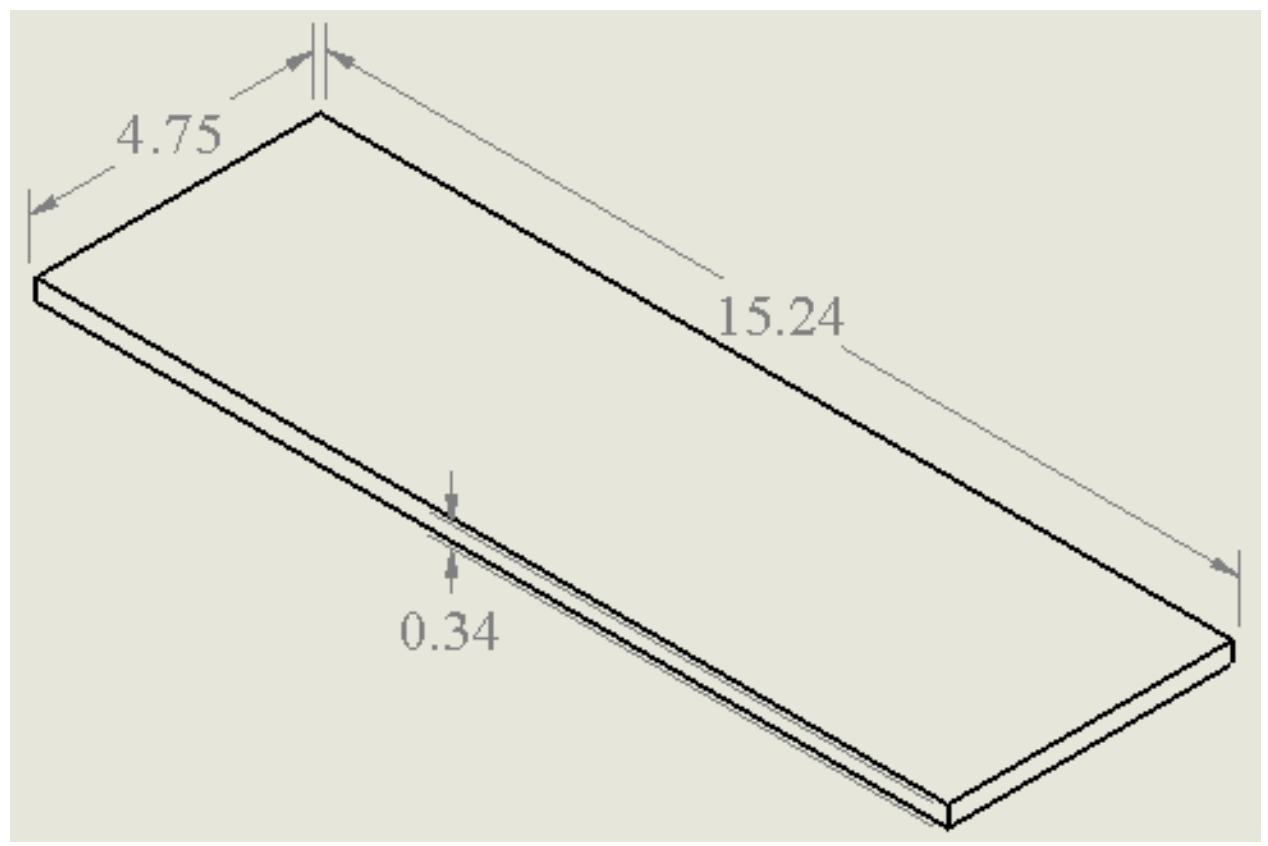

Figure A.5 Terminator splitter plate [mm]

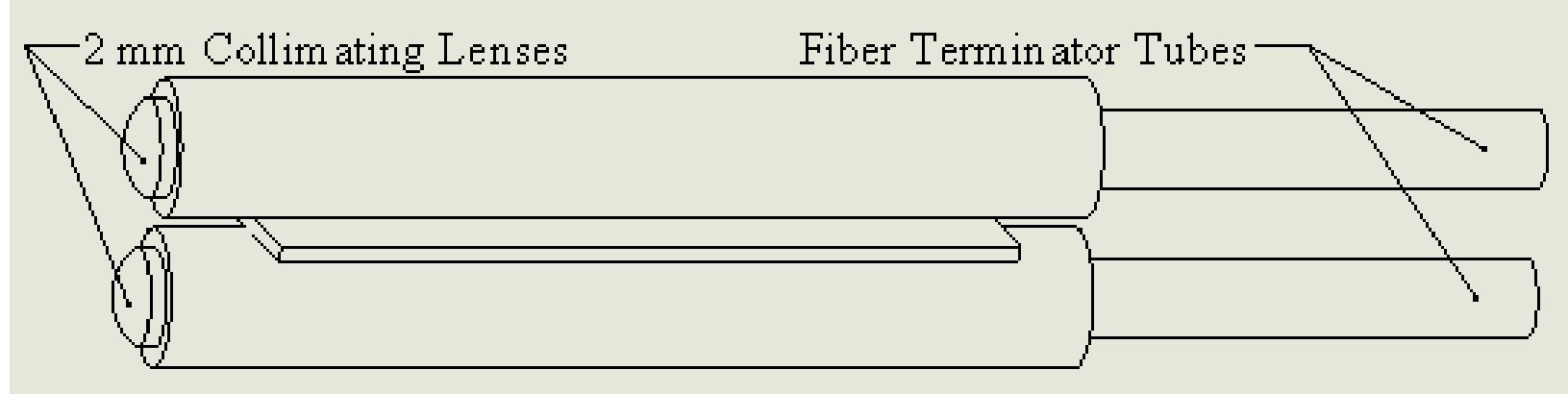

Figure A.6 Transimitting assembly 


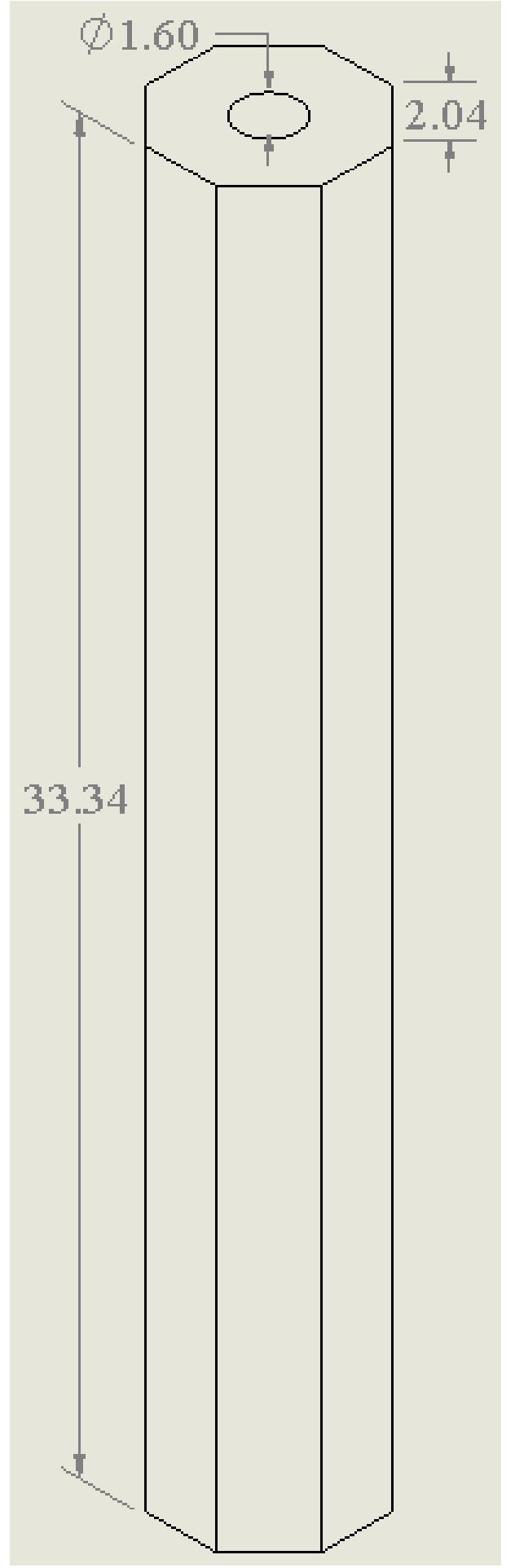

Figure A.7 Receiving optics holder [mm]

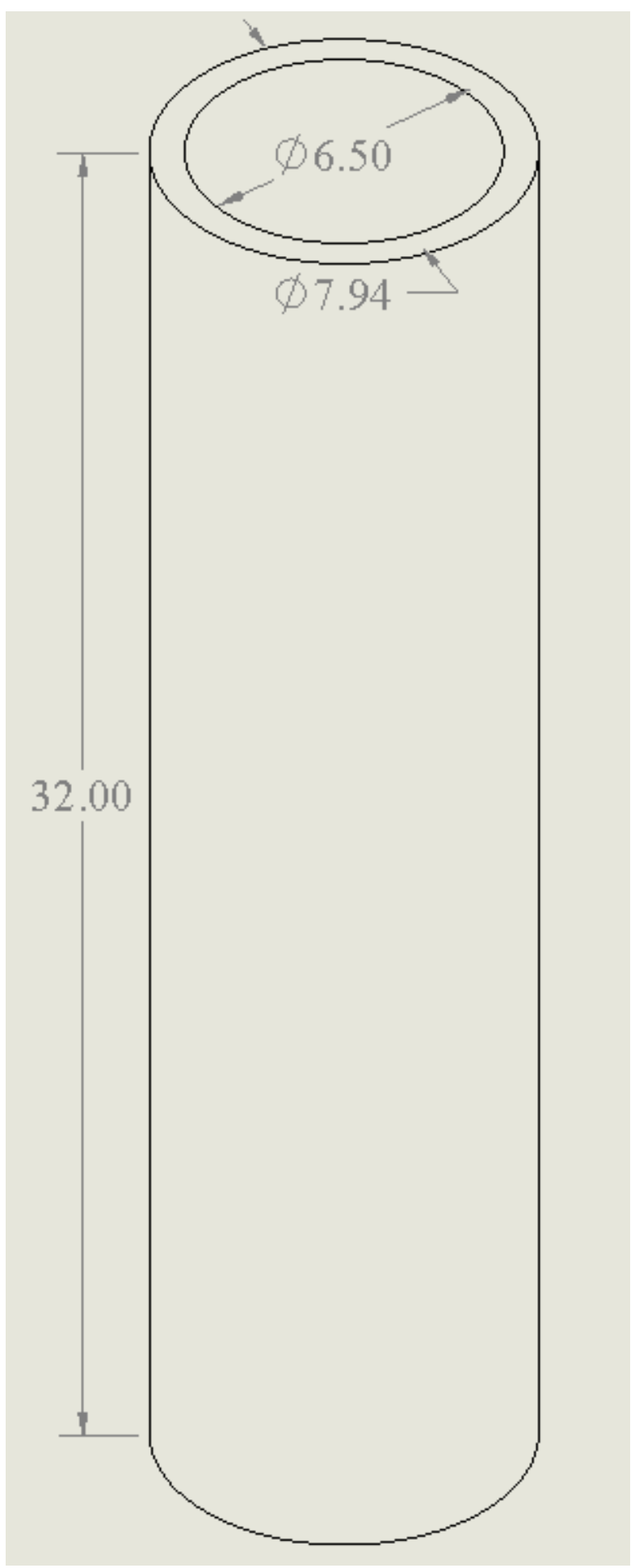

Figure A.8 Receiving optics tube [mm] 


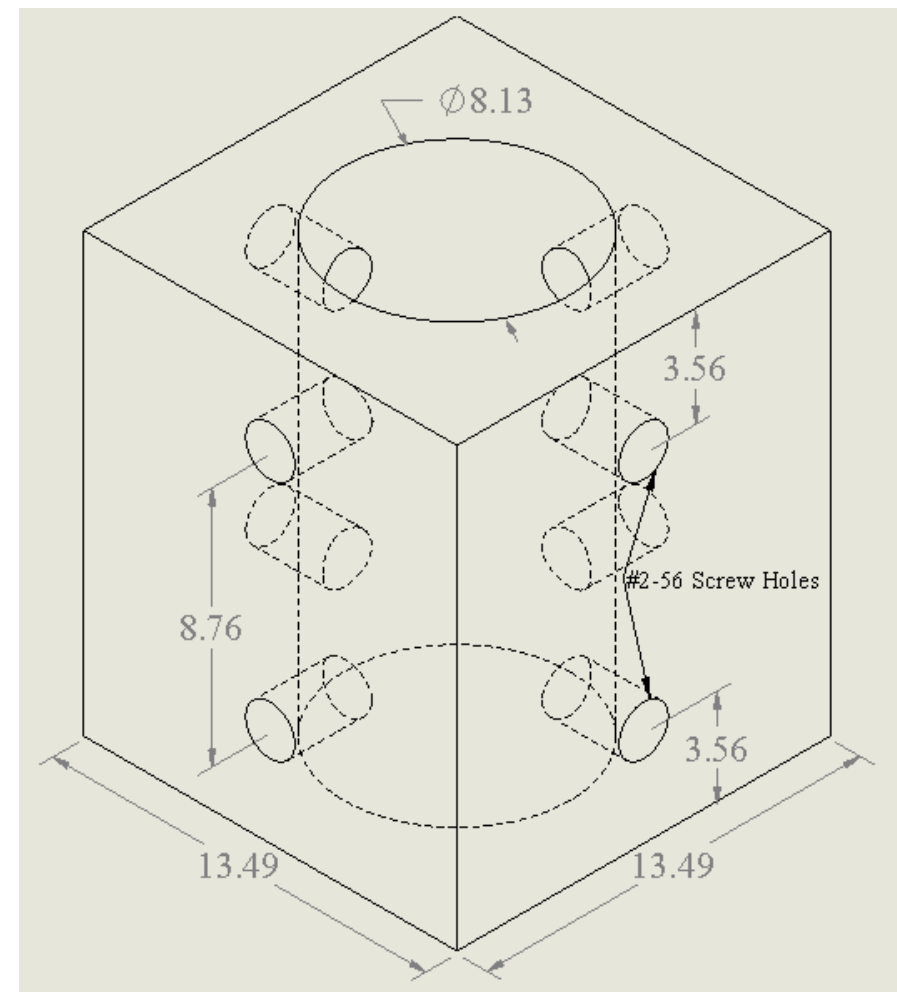

Figure A.9 Receiving optics cube [mm]

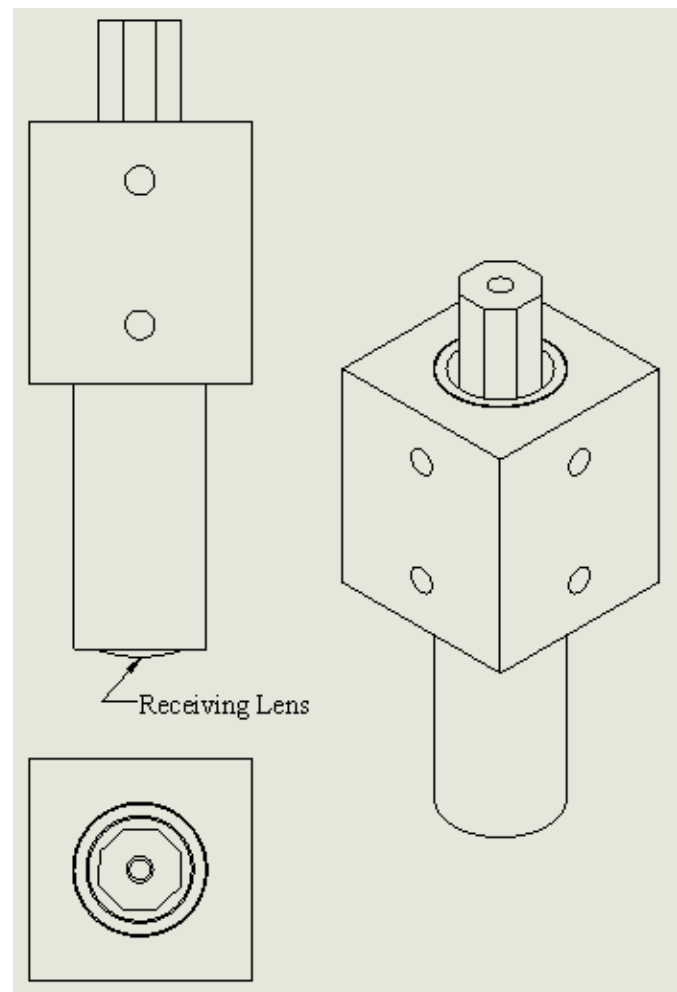

Figure A.10 Receiving optics assembly 


\section{B. DISCRETE FOURIER TRANSFORM}

The continuous Fourier transform is defined as

$$
\begin{aligned}
f(v) \quad & =\mathcal{F}_{t}[f(t)](v) \\
& =\quad \int_{-\infty}^{\infty} f(t) e^{-2 \pi i v t} d t .
\end{aligned}
$$

Now consider generalization to the case of a discrete function, $f(t) \rightarrow f\left(t_{k}\right)$ by letting $f_{k} \equiv f\left(t_{k}\right)$, where $t_{k} \equiv k \Delta$, with $k=0, \ldots, N-1$. Writing this out gives the discrete Fourier transform $F_{n}=\mathcal{F}_{k}\left[\left\{f_{k}\right\}_{k=0}^{N-1}\right](n)$ as

$$
F_{n} \equiv \sum_{k=0}^{N-1} f_{k} e^{-2 \pi i n k / N}
$$

The inverse transform $f_{k}=\mathcal{F}_{n}^{-1}\left[\left\{F_{n}\right\}_{n=0}^{N-1}\right](k)$ is then

$$
f_{k}=\frac{1}{N} \sum_{n=0}^{N-1} F_{n} e^{2 \pi i k n / N} .
$$

Discrete Fourier transforms are extremely useful because they reveal periodicities in input data as well as the relative strengths of any periodic components. There are a few subtleties in the interpretation of discrete Fourier transforms, however. In general, the discrete Fourier transform of a real sequence of numbers will be a sequence of complex numbers of the same length. In particular, if $f_{k}$ are real, then $F_{N-n}$ and $F_{n}$ are related by

$$
F_{N-n}=\bar{F}_{n},
$$

for $n=0,1, \ldots, N-1$, where $\bar{z}$ denotes the complex conjugate. This means that the component $F_{0}$ is always real for real data. 
As a result of the above relation, a periodic function will contain transformed peaks in not one, but two places. This happens because the periods of the input data become split into "positive" and "negative" frequency complex components.

Reference:

Weisstein, Eric W. "Discrete Fourier Transform." From MathWorld--A Wolfram Web Resource. http://mathworld.wolfram.com/DiscreteFourierTransform.html 


\section{CUNNINGHAM SLIP CORRECTION FACTOR}

The Cunningham slip correction factor accounts for noncontinuum effects when calculating the drag on small particles. At high Knudsen numbers, the no-slip condition is no longer valid and the correction factor allows for predictiong the drag force on a particle moving a fluid with Knudsen number between the continuum regime and free molecular flow.

The drag coefficient, calculated as usual, is simply divided by the correction factor. Equation C.1 gives the Cunninham slip correction factor.

$$
C=1+\frac{2 \lambda}{d}\left(A_{1}+A_{2} e^{\frac{-A_{3} d}{\lambda}}\right)
$$

where $C$ is the correction factor, $\lambda$ is the mean free path, $d$ is the particle diameter and, $A_{n}$ are experimentally determined coefficients. For air, $A_{1}=1.257, A_{2}=0.400$ and, $A_{3}=0.55$. The correction factor becomes significant when particles become smaller that 15 micrometers, for air at ambient conditions.

\section{Reference:}

Cunningham, E. 1910. On the Velocity of Steady Fall of Spherical Particles through Fluid Medium. Proceedings of the Royal Society, Vol 83, 357. 


\section{COANDA EFFECT}

The Coanda effect is the phenomena in which a jet flow attaches itself to a nearby surface and remains attached even when the surface curves away from the initial jet direction. A jet of fluid in free surroundings entrains and mixes with its surroundings as it flows away from a nozzle.

A surface brought close to the jet restricts the entrainment in that region and as flow accelerates to try to balance the momentum transfer, a pressure difference across the jet results and the jet is deflected closer to the surface and eventually attaches to it.

Even if the surface is curved away from the initial direction, the jet tends to remain attached. This effect can also be used to change the direction of the jet. The rate at which the jet mixes is often significantly increased compared with that of an equivalent free jet.

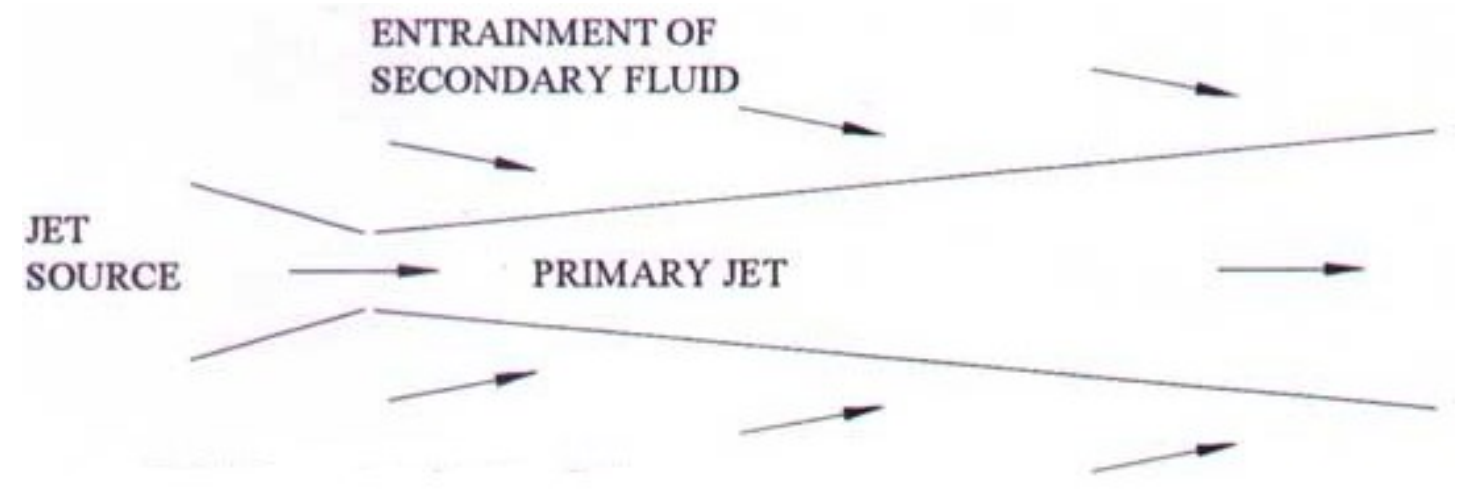

Figure D.1 Free jet flow 


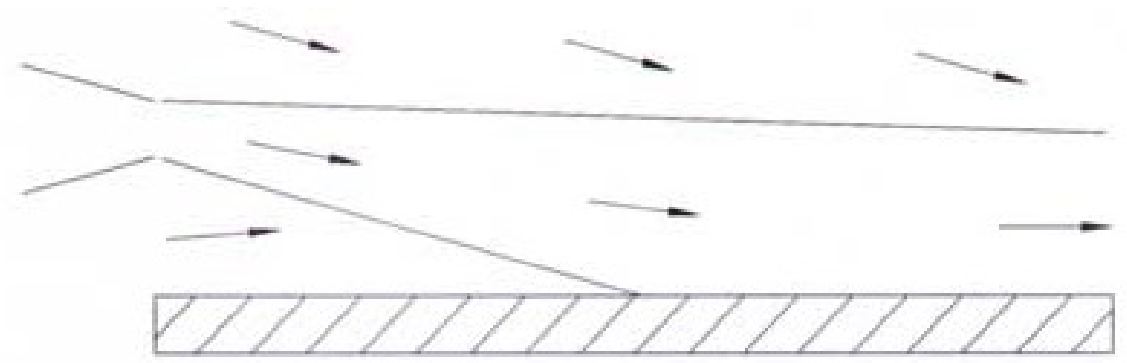

Figure D.2 Jet attaching to adjacent surface

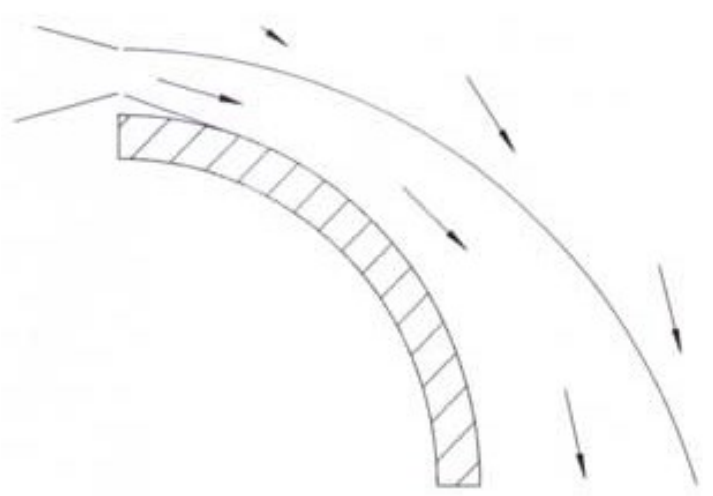

Figure D.3 Jet attaching to and turning with curved surface 